\title{
Manta ray foraging and Gaussian mutation-based elephant herding optimization for global optimization
}

\author{
Yuxian Duan ${ }^{1,2} \cdot{\text { Changyun } \mathrm{Liu}^{1} \cdot \text { Song } \mathrm{Li}^{1} \cdot \text { Xiangke Guo }^{1} \cdot \text { Chunlin Yang }}^{2,3}$
}

Received: 2 April 2021 / Accepted: 22 July 2021

(C) The Author(s) 2021

\begin{abstract}
The elephant herding optimization (EHO) algorithm is a novel metaheuristic optimizer inspired by the clan renewal and separation behaviors of elephant populations. Although it has few parameters and is easy to implement, it suffers from a lack of exploitation, leading to slow convergence. This paper proposes an improved EHO algorithm called manta ray foraging and Gaussian mutation-based EHO for global optimization (MGEHO). The clan updating operator in the original EHO algorithm is replaced by the somersault foraging strategy of manta rays, which aims to optimally adjust patriarch positions. Additionally, a dynamic convergence factor is set to balance exploration and exploitation. The gaussian mutation is adopted to enhance the population diversity, enabling MGEHO to maintain a strong local search capability. To evaluate the performances of different algorithms, 33 classical benchmark functions are chosen to verify the superiority of MGEHO. Also, the enhanced paradigm is compared with other advanced metaheuristic algorithms on 32 benchmark functions from IEEE CEC2014 and CEC2017. Furthermore, a scalability test, convergence analysis, statistical analysis, diversity analysis, and running time analysis demonstrate the effectiveness of MGEHO from various aspects. The results illustrate that MGEHO is superior to other algorithms in terms of solution accuracy and stability. Finally, MGEHO is applied to solve three real engineering problems. The comparison results show that this method is a powerful auxiliary tool for handling complex problems.
\end{abstract}

Keywords Metaheuristic algorithm · Elephant herding optimization · Manta rays optimization · Benchmark function · Swarm intelligence

\section{Introduction}

Optimization has long been a fundamental tool for applications in mathematics, medicine, engineering, the military, and many other fields. In general, optimization is an approach used to determine the best performance of a system that can be achieved based on optimized parameters to make the final solution as perfect and efficient as possible [1]. In optimization problems, the final goal is usually

Song $\mathrm{Li}$

songli1126@163.com

$1 \quad$ Air and Missile Defense College, Air Force Engineering University, Xi'an 710051, China

2 Graduate College, Air Force Engineering University, Xi' an 710051, China

3 Air Traffic Control and Navigation College, Air Force Engineering University, Xi'an 710051, China considered the maximization or minimization of some objective function (the function used to evaluate the nature or quality of the resulting solution). In this case, several steps are involved, such as defining a mathematical model for the system, identifying variable, defining the type of system needed, specifying constraints, and then finding the optimization response [2].

Traditional optimization techniques include the dynamic programming approach (DPA), stochastic search, the steepest descent method, and Newton's method. Most of these methods are nonpolynomial and can find optimal solutions smoothly. Unfortunately, they are usually limited by the specific problem and the size of the given dataset. Furthermore, they are often highly complex and specialized in nature. In addition, the execution times and running costs are very high [3]. Therefore, such approaches are only suitable for solving small-scale problems. Currently, most real-world optimization problems are complex, highly 
nonlinear, and large-scale spatial, and the information metrics used are essentially multifaceted. On this basis, traditional methods are not suitable for such situations. The popular trend is to combine the multifaceted nature of the subject system with various proposed heuristics to solve difficult problems. Heuristics methods include nearest neighbor algorithms, evolutionary algorithms, insertion algorithms, memetic algorithms, dynamic relaxation, etc. By introducing greedy mechanisms, the number of search steps is fixed, and the number of iterations is reduced. With these advantages, heuristic methods are able to find nearoptimal solutions with less time costs than those of other methods [4]. However, most of them are designed for specific problems. Due to the introduction of greedy mechanisms, these methods often fall into local optima when solving complex problems. Soon after the development of heuristic methods, metaheuristics, which are problem-independent adaptive strategies, emerged to provide a set of guidelines or strategies for developing heuristics. In this term, "meta" means using a higher level, usually one level higher than that of the given heuristic, to solve a variety of intractable and complex hard problems [5]. Specifically, metaheuristics perform the search process by using multiple agents, which are essentially systems that find the best solution based on constraints and rules over multiple iterations. The iterative process is stopped when a predefined criterion is satisfied, at which point the solution found is determined to be the best solution and the system reaches a fused state. Metaheuristics are not dependent on gradient information, are easy to execute and are highly flexible because they treat the optimization process as a black box [6]. As research has progressed, metaheuristics have been developed as powerful tools and are now widely used in various fields.

In general, metaheuristic algorithms are classified into four categories, namely, evolutionary-based algorithms, physics-based algorithms, population-based algorithms, and human-based algorithms. Inspired by Darwinian evolutionary theory, evolutionary-based algorithms were introduced in the early 1970s and usually draw from natural rules to find optimal solutions. By setting one or more operators (crossover, variation, or selection operators), such algorithms evaluate search agents during optimization [7]. Evolutionary-based algorithms include genetic algorithms (GA) [8], biogeography-based optimization algorithms (BBO) [9], differential evolutionary algorithms (DE) [10], etc. Physics-based algorithms are modelled according to physical rules. For example, the gravitational search algorithm (GSA) [11] and the equilibrium optimizer (EO) [12] are all physics-based approaches. Populationbased algorithms simulate the group behaviour of animals, performing hunting based on cooperation. In populationbased algorithms, multiple agents perform the search process together and share useful information with each other, thus improving operational efficiency. Representative algorithms include the artificial bee colony (ABC) algorithm [13], the sailfish optimizer (SFO) [14], whale optimization algorithm (WOA) [15], Harris hawks algorithm (HHO) [16], etc. Human-based algorithms generally simulate the special behaviour of humans. Such algorithms include the harmony search algorithm (HS) [17], teachinglearning-based optimization (TLBO) [18].

Elephant herding optimization (EHO) is a populationbased algorithm proposed by Wang et al. [19] . Inspired by the nomadic herding behaviour of elephant populations, EHO is designed to solve global optimization problems. In $\mathrm{EHO}$, an entire population is divided into different clans. Each clan is led by a female matriarch, while the male elephants live independently when they reach adulthood. EHO sets up two operators, a clan updating operator and a separation operator, to update the positions of the agents. A clan updating operator is implemented for each individual, and the next position of each individual is updated according to the current position in relation to the position of the clan leader. Subsequently, the agent with the worst position is replaced by the separation operator to speed up convergence and enhance the diversity of the population. In terms of advantages, EHO has fewer controlled parameters, is easier to implement, and has better performance than other optimization approaches, so it attracts much attention from mathematicians and engineers [20].

In response to the shortcomings of EHO, researchers have proposed many improvement ideas and optimization methods. For instance, Muthusamy et al. [21] introduced the sine cosine algorithm (SCA) and opposition-based learning (OBL) to update the positions of agents in the original EHO algorithm. Through this operation, the convergence speed and the searchability of the algorithm were enhanced. Li and Wang [20] proposed a new improved EHO approach based on dynamic topology and BBO methods. The operators of this EHO variant were updated, and a new separation operator was set, thus ensuring that the evolutionary process of the examined population moved in a better direction. Ismaeel et al. [22] proposed three versions of an unbiased algorithm, EEHO15, EEHO20, and EEHO25, to overcome the defect of unreasonable convergence in the original EHO algorithm. Three similar variants were proposed by Elhosseini et al. [23], namely cultural-based, alpha-tuning, and biased initialization EHO, with the aim of strengthening the global optimization capability of EHO. Balamurugan et al. [24] proposed an opposition-based EHO (OEHO) algorithm. In their work, opposition-based learning was adopted to enhance the performance of EHO. Xu et al. [25] proposed a novel algorithm, LFEHO, which combined Levy flight with EHO to overcome the problems of the original EHO 
algorithm falling into local optima and exhibiting poor convergence performance. Improved EHO algorithms such as IMEHO were presented by $\mathrm{Li}$ et al. [26] The robustness and diversity of a given population are optimized by using a new global learning-based evolution strategy to update the velocities and positions of agents. Reference [27] extended and improved the original EHO algorithm by designing six strategies to update the agents. The information obtained from the previous iterations of individuals was adopted for weighing operations. Therefore, the obtained results were better than those output by the original EHO algorithm. In addition, engineers extended the application of EHO to solve practical engineering problems in real production cycles.

In addition, MRFO is a novel optimization algorithm proposed by Zhao et al. [28]. It is a promising and powerful optimizer inspired by the predatory behaviour of manta rays. MRFO has three foraging operators, namely, chain foraging, cyclone foraging, and tumble foraging operators. Recently, researchers have introduced MRFO to other fields. For example, Ekinci et al. [29] combined the opposition-based (OBL) with MRFO and hybrid simulated annealing algorithm to provides MRFO a better exploration capability. Shaheen et al. [30] optimized the MRFO algorithm by using adaptive penalty parameters and introduced it to handle a thermal scheduling problem. In addition, MRFO has been extended to many fields, such as feature selection (FS) [31], fuel power generation [32], system identification [33], photovoltaic system operation [34], and other areas.

According to the no-free lunch (NFL) theorem [35], any algorithm that is applicable for solving a given problem cannot provide the best solution for all problems. In other words, there is a need to improve the metaheuristic algorithms that exist in reality. Maintaining a balance between exploration and exploitation is the common and fundamental feature of any optimization algorithm and the most challenging problem in metaheuristics. Among them, exploration is the process of exploring optimal solutions over a wide range of unknown regions, while the exploitation of explored regions allows an algorithm to effectively find high-quality solutions. Therefore, it is necessary to fine-tune exploration and exploitation, as they are the two main factors used to obtain satisfactory results. In addition, it has been shown that although some algorithms are very effective in solving certain types of problems or large-scale problems, more significant optimization results can be achieved by combining the advantages of different algorithms in a hybrid manner [36]. By combining them, the convergence performance of the resulting hybrid algorithm is improved and the balance between exploration and exploitation is better maintained, thus allowing the algorithm to avoid falling into local optima. The above facts have motivated researchers to continuously develop hybrid metaheuristic algorithms with good exploration and exploitation capabilities and have made our research highly relevant.

Like most metaheuristic algorithms, EHO is prone to falling into local optima when dealing with complex multipeaked problems, which stems from the stochasticity of such problems and the imbalance between exploration and exploitation. Therefore, these shortcomings become major issues that affect the performance of EHO. At the same time, EHO also has drawbacks such as a poor exploitation capability and a low convergence rate. To summarize, there are three defects in the EHO algorithm that need to be solved.

(a) The position of the female matriarch is determined by all members of the clan. Under this circumstance, once the algorithm falls into a local optimum, the matriarch will lose her ability to explore.

(b) The location update strategy for individual elephants is fixed and lacks a certain control mechanism for adjustments according to the iterative process. Therefore, it is difficult to strike a balance between exploration and exploitation when dealing with complex problems.

(c) In addition, the separation operator does not guarantee that the worst individual in the clan is updated to a better position. To address the shortcomings of EHO, a manta ray foraging and Gaussian mutationbased EHO algorithm for global optimization called MGEHO is proposed. The contributions of this paper are summarized as follows:

1. The position of search agents in EHO is optimized by hybridizing the tumbling foraging strategy in MRFO, which improves the ability of the original EHO algorithm to jump out of local optima. Additionally, the global optimization ability of the original algorithm is enhanced.

2. To maintain a balance between exploration and exploitation, a nonlinear dynamic convergence factor is set to improve the clan updating operator in EHO and the Somersault foraging in MRFO. On this basis, the global exploration and local exploitation processes can be coordinated.

3. Furthermore, Gaussian mutation is introduced to adjust the position of the worst individual of each clan. By using random numbers conforming to a Gaussian distribution instead of the original model parameters, new solutions in the vicinity of the candidate solutions are generated, and the diversity of the population is enhanced. 
4. To fully validate its effectiveness, MGEHO is tested on 33 benchmark functions and compared with 9 other well-known metaheuristics. Furthermore, performance tests are conducted on the algorithm in different dimensions. Moreover, nonparametric statistical tests are set up to highlight the differences between MGEHO and other competing approaches. A paired Wilcoxon symbolic rank test and a Friedman test at the 5\% significance level are invoked to test the compared algorithms. Besides, diversity tests of MGEHO and EHO are performed to verify the improved performance on exploration and exploitation.

5. The performance of the proposed MGEHO is also evaluated and compared with selected advanced algorithms by using 32 modern CEC2014 and CEC2017 benchmark functions. Statistical tests are invoked to analyze the results of means and standard deviations. The Holm test is used as a post hoc test. In addition, the running time analysis is conducted to highlight the fundamental differences between the proposed and existing methods.

6. In addition, the proposed MGEHO algorithm is used to solve three practical engineering problems: (a) a design problem regarding a gear train; (b) a design problem with a three-bar truss; and (c) a design problem with respect to a pressure vessel. The experimental results show that MGEHO has superior adaptability and the ability to handle optimization problems.

The rest of the paper is organized as follows: Sect. 2 reviews the original EHO and MRFO algorithms. Section 3 provides a detail of the proposed MGEHO method. Experiments and the analysis of the results are given in Sect. 4. Tests on practical engineering problems are conducted in Sect. 5. Section 6 summarizes the conclusions of this paper and plans for future work.

\section{Preliminary}

\subsection{EHO}

In general, elephants are gregarious creatures. Within a population, they are divided into different clans. Individual elephants from the same clan live together under the leadership of a female matriarch. After reaching adulthood, a fixed number of males leave the clan. EHO simulates these characteristics of elephant herd life by dividing the behaviours of searching agents into two parts, namely, a clan updating operator and separation operator.

\subsubsection{Clan updating operator}

The position of an elephant individual $j$ in clan $C_{i}$ is updated in the following way:

$x_{\text {new }, \mathrm{c}_{\mathrm{i}} \mathrm{j}}=x_{c_{i}, j}+\alpha \times\left(x_{\text {best }, \mathrm{c}_{\mathrm{i}}}-x_{c_{i}, j}\right) \times r$,

where $x_{\text {new }, \mathrm{c}_{\mathrm{i}}, \mathrm{j}}$ and $x_{c_{i}, j}$ are the new and original positions of elephant individual $j$ in clan $C_{i}$, respectively. $\alpha$ and $r$ are both random numbers within $[0,1]$. In addition, $x_{\text {best, } c_{\mathrm{i}}}$ is the best individual of clan $C_{i}$, which is updated by the following equation:

$$
\begin{aligned}
& x_{\text {new }, \mathrm{c}_{\mathrm{i}}, \mathrm{j}}=\beta \times x_{\text {center }, \mathrm{c}_{\mathrm{i}}}, \\
& x_{\text {center }, \mathrm{c}_{\mathrm{i}}}=\frac{1}{n_{c_{i}}} \times \sum_{j=1}^{n_{c_{i}}} x_{c_{i}, j},
\end{aligned}
$$

where $x_{\text {center, } \mathrm{c}_{\mathrm{i}}}$ represents the central positions of all individuals in clan $C_{i}$ and $n_{c_{i}}$ stands for the number of elephants in clan $C_{i} . \beta$ affects the extent to which $x_{\text {center, } \mathrm{c}_{\mathrm{i}}}$ acts on $x_{\text {new }, \mathrm{c}_{\mathrm{i}}, \mathrm{j}}, \beta \in[0,1]$.

\subsubsection{Separation operator}

A separation operator is proposed to update the worst individual in each clan by the following equation:

$x_{\text {worst }, \mathrm{c}_{\mathrm{i}}}=x_{\min }+\left(x_{\max }-x_{\min }+1\right) \times r$,

where $x_{\max }$ and $x_{\min }$ are the upper and lower bounds of the individual positions, respectively. $r$ is a random number within $[0,1]$.

\subsubsection{Pseudocode of EHO}

In general, the pseudocode of EHO is shown in Algorithm 1. 


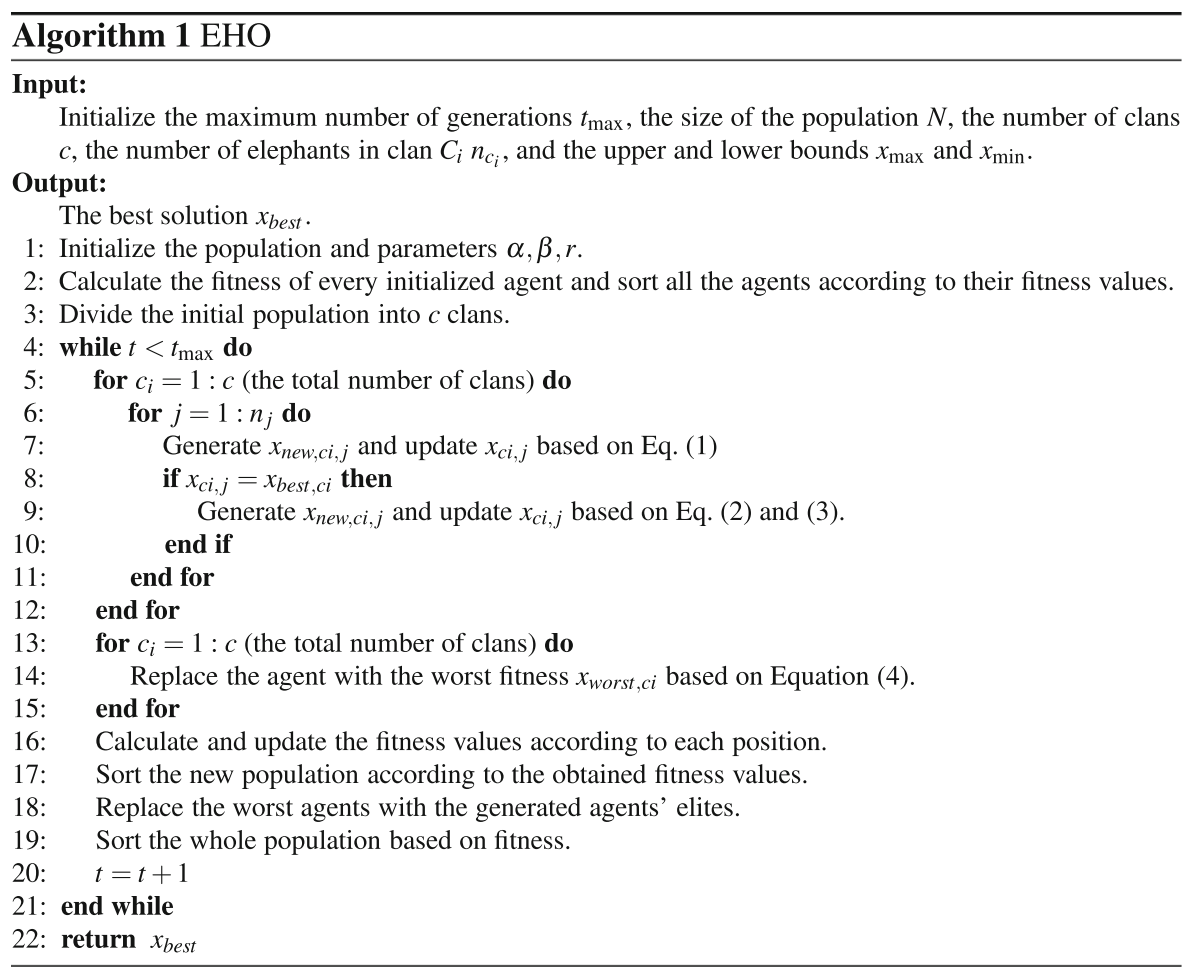

\subsection{MRFO}

In MRFO, three manta ray foraging strategies are simulated, namely chain foraging, cyclone foraging, and somersault foraging. Similar to other metaheuristics, MRFO also performs initialization first and then updates each agent sequentially through iteration to find the best solution.

\subsubsection{Chain foraging}

In the first stage, manta rays form a foraging chain sequentially from front to back, and the position of each search agent is updated by the current optimal solution and the solutions generated during previous iterations. For a set of individuals $x_{i}$, the process of position updating is represented as follows: where $x_{i}^{d}(t)$ represents the position of $i$ th individual at time $t$ in the $d$ th dimension. $r$ represents a random number within [0,1]. $N$ is the size of the population. $x_{\text {best }}^{d}$ is the $\alpha$ stands for a weight coefficient determined in the following way:

$$
\alpha=2 \times r \times \sqrt{|\log (r)|} .
$$

\subsubsection{Cyclone foraging}

During this stage, manta rays swim forward in a spiral pattern. Unlike the spiral foraging approach in the WOA, each individual not only approaches its prey along the spiral path but also adjusts to the positions of the current and previous best agents and keeps swimming forward. The mathematical model for this process is represented as

$$
x_{i}^{d}(t+1)=\left\{\begin{array}{cc}
x_{i}^{d}(t)+(r+\alpha) \times\left[x_{\text {best }}^{d}(t)-x_{i}^{d}(t)\right] & i=1 \\
x_{i}^{d}(t)+r \times\left[x_{i-1}^{d}(t)-x_{i}^{d}(t)\right]+\alpha \times\left[x_{\text {best }}^{d}(t)-x_{i}^{d}(t)\right] & i=2, \ldots \ldots N
\end{array}\right.
$$


$x_{i}^{d}(t+1)=\left\{\begin{array}{cc}x_{\text {best }}^{d}+(r+\beta) \times\left[x_{\text {best }}^{d}(t)-x_{i}^{d}(t)\right] & i=1 \\ x_{\text {best }}^{d}+r \times\left[x_{i-1}^{d}(t)-x_{i}^{d}(t)\right]+\beta \times\left[x_{\text {best }}^{d}(t)-x_{i}^{d}(t)\right] & i=2, \ldots \ldots . N\end{array}\right.$.

follows:

The weight coefficient $\beta$ is expressed as follows:

$\beta=2 e^{r_{1}\left(\frac{T-t+1}{T}\right)} \times \sin \left(2 \pi r_{1}\right)$,

where $r_{1}$ is a random number, $r_{1} \in[0,1]$. $T$ denotes the maximum number of iterations. In addition, agents at this stage update their positions by searching for random positions in the space to enhance their exploration abilities in the following manner:

$x_{i}^{d}(t+1)=$

$\left\{\begin{array}{cc}x_{\text {rand }}^{d}+(r+\beta) \times\left[x_{\text {rand }}^{d}-x_{i}^{d}(t)\right] & i=1 \\ x_{\text {rand }}^{d}+r \times\left[x_{i-1}^{d}(t)-x_{i}^{d}(t)\right]+\beta \times\left[x_{\text {rand }}^{d}-x_{i}^{d}(t)\right] & i=2, \ldots \ldots N\end{array}\right.$,

where $x_{\text {rand }}^{d}$ denotes a random position in dimension $d$ and $x_{\text {rand }}^{d}=x_{\min }^{d}+r \times\left(x_{\max }^{d}-x_{\min }^{d}\right) . x_{\max }$ and $x_{\min }$ represent the upper and lower bounds of the search space, respectively.

\subsubsection{Somersault foraging}

In somersault foraging, the MRFO algorithm treats food as a hub, and each agent makes a flipping motion along the hub. On this basis, agents can update their positions around the current optimal solution to enhance their exploitation capabilities. The mathematical model of this process is shown as follows:

$$
\begin{aligned}
& x_{i}^{d}(t+1)=x_{i}^{d}(t)+S \times\left[r_{2} \times x_{\text {best }}^{d}(t)-r_{3} \times x_{i}^{d}(t)\right] \\
& \quad i=1,2, \ldots \ldots N
\end{aligned}
$$

where $S$ is the somersault factor, which is used to indicate the range of each flip. In MRFO, the parameter is set to 2 . $r_{2}$ and $r_{3}$ are both random numbers within $[0,1]$.

\subsubsection{Pseudocode of MRFO}

The pseudocode of MRFO is shown in Algorithm 2.

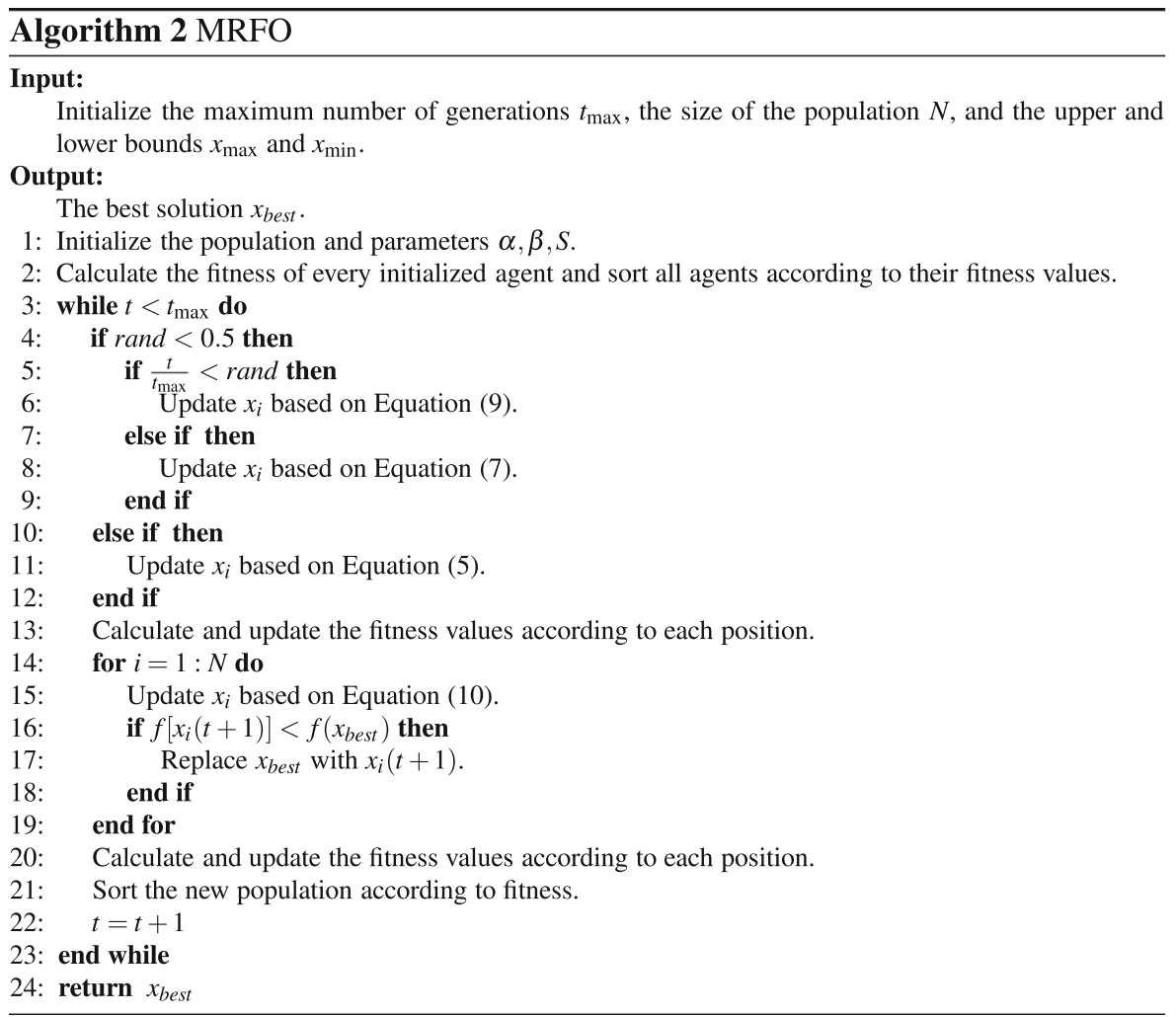




\section{The proposed method}

In $\mathrm{EHO}$, the position update strategies of the clan leader and other members are different. Specifically, the position of the patriarch is determined by the action factor $\beta$ and the central positions of all agents in the same clan during each iteration. When $\beta$ is small, the patriarch moves near the origin. When $\beta$ is large, the patriarch suddenly moves to the center of the clan. This kind of strategy lacks guidance towards the global optimal solution, so the algorithm may fall into a local optimum, which affects its convergence efficiency and reduces its exploration ability. Second, the search process of EHO is fixed and lacks an effective way to control the balance between exploration and exploitation, and this largely affects the performance of the algorithm. Third, the worst individuals in the clan are randomly replaced by the separation operator, which makes it difficult to ensure the improvement of population diversity.

Therefore, MGEHO is proposed for the purpose of improving the above shortcomings of EHO. In this section, three aspects of the newly developed strategy are explained in detail.

\subsection{Hybrid MRFO with EHO}

First, we update the clan updating operator in EHO. Specifically, the somersault foraging strategy is introduced to update the position of the patriarch in each clan, as shown in Fig. 1. According to this novel behaviour, the patriarch can be updated to a position symmetric to the pivot point with the global optimal solution as the pivot point. As the distance between the position of each agent and that of the optimal solution decreases, the fluctuation of the current position decreases, and the range of somersault foraging decreases. Unlike opposition-based learning, somersault foraging is centered around the global optimal

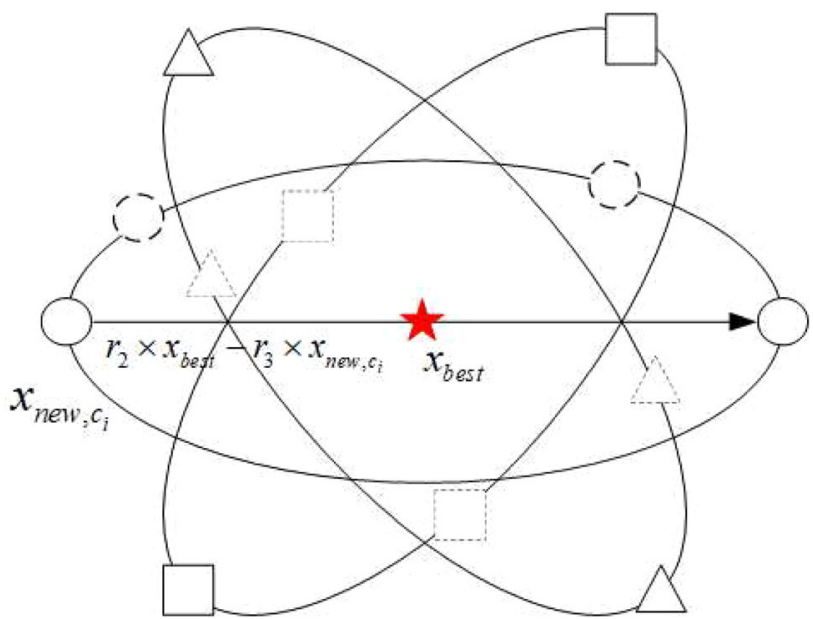

Fig. 1 Somersault foraging strategy solution, so it is more directional, and the convergence rate of the algorithm is further enhanced. An agent that falls into a local optimum can be compared with its updated agent so that it can jump out of the local optimum.

Second, after finishing the clan updating and separation operations, chain foraging and cyclone foraging are executed. Later, the elite agents are selected for retention by comparing the fitness values of the agents generated in the $i$ th iteration.

\subsection{Nonlinear convergence factor}

In the original EHO algorithm, there is a lack of effective control over diversity and convergence, which limits the ability of the algorithm to find the best convergence rate. For this reason, a nonlinear convergence factor is introduced that can change dynamically with the iterative process. In the early iterations of the algorithm, the weights are relatively large, which can enhance the global search ability and diversity of the population. In later iterations, the weights decrease so that the algorithm can focus on the explored areas, improve the local search ability, and accelerate the convergence rate. The convergence factor is expressed as follows:

$\omega=\omega_{l}+\omega_{u} \times e^{-2 k \sin \left(\frac{t}{t_{\max }}\right)}$,

where $\omega_{u}$ and $\omega_{l}$ are the upper and lower bounds of the convergence factor, respectively. $k$ is the impact factor. When $k$ takes different values, the corresponding changes in the convergence factor are shown in Fig. 2. In the early iterations, the convergence factor is larger and scales down more, which will allow the population to perform a broad exploration. In later iterations, the convergence factor decreases and slows, which allows MGEHO to perform a

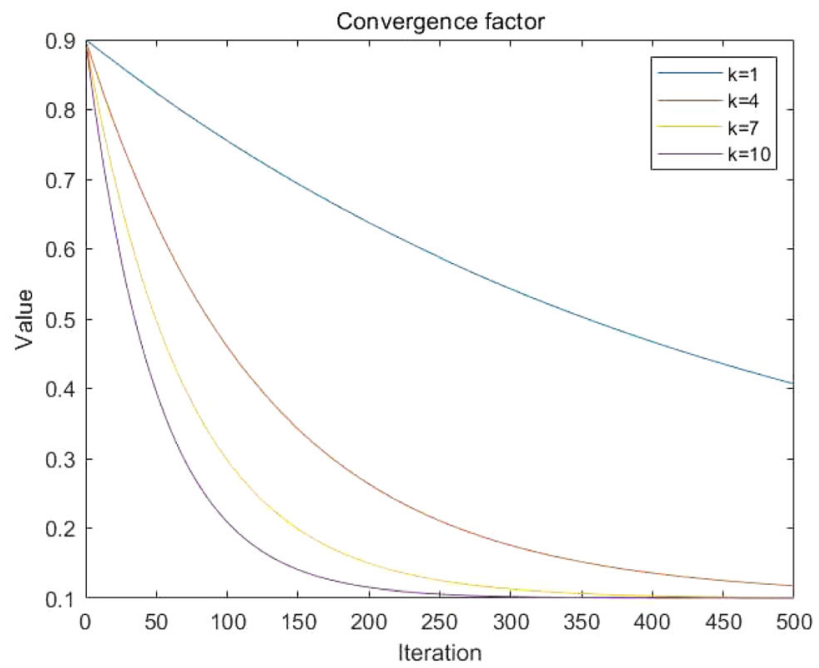

Fig. 2 Variation curves of the convergence factor 
fine local search. As observed in Fig. 2, when the impact factor $k$ changes, $\omega$ decreases in a nonlinear manner, and the rate of decrease gradually accelerates as the impact factor increases. The choice of the influencing factor depends on the choices and requirements of the specific problem. On this basis, the position of elephant individual $j$ in clan $C_{i}$ is updated in the following way:

$x_{\text {new }, \mathrm{c}_{\mathrm{i}} \mathrm{j}}=\omega \times x_{c_{i}, j}+\alpha \times\left(x_{\text {best }, \mathrm{c}_{\mathrm{i}}}-x_{c_{i}, j}\right) \times r$.

In addition, the position of the patriarch in each clan is refreshed in the following manner:

$$
\begin{aligned}
x_{\text {new, },_{\mathrm{i}}}^{d}(t+1) & =\omega \times x_{\text {new, } \mathrm{c}_{\mathrm{i}}}^{d}(t)+S \\
& \times\left[r_{2} \times x_{\text {best }}^{d}(t)-r_{3} \times x_{\text {new }, \mathrm{c}_{\mathrm{i}}}^{d}(t)\right] .
\end{aligned}
$$

\subsection{Gaussian mutation strategy}

Gaussian mutation [37] was proposed by Back and Schwefe to improve the search efficiency of heuristic algorithms. Generally, Gaussian mutation generates new solutions in the vicinity of the candidate solutions. It can explore every corner of the search space in small steps during the execution of the search, thus enhancing the population diversity. As a successful strategy, it has been widely used in algorithms such as the GA and PSO [38].
When performing the mutation operation, the original values are replaced with eligible random numbers as the mean and variance of a Gaussian distribution [39]. The expression of the Gaussian distribution function is as follows:

$f_{\left(0, \sigma^{2}\right)}(\delta)=\frac{1}{\sqrt{2 \pi \sigma^{2}}} e^{\frac{-\delta^{2}}{2 \sigma^{2}}}$,

where $\sigma^{2}$ is the variance value of the candidate solutions. $\delta$ represents the mean value. After applying a Gaussian distribution to MGEHO, the worst individual position of each clan is updated to explore a larger spatial region and increase the diversity of the candidate solutions. The equation for applying the Gaussian distribution is shown as follows:

$\hat{x}_{i}=x_{i} \times[1+\operatorname{Gaussian}(\delta)]$,

where Gaussian $(\delta)$ is a Gaussian distributed random variable and $x_{i}$ represents the $i$ th candidate solution.

\subsection{Pseudocode of MGEHO}

The pseudocode of MGEHO is shown in Algorithm 3.
Table 1 Details of the unimodal benchmark functions

\begin{tabular}{lllll}
\hline No. & Function & Dimensions & Range & $f_{\text {min }}$ \\
\hline F1 & $f_{1}(x)=\sum_{i=1}^{n} x_{i}^{2}$ & $30,100,200$ & {$[-100,100]$} & 0 \\
F2 & $f_{2}(x)=\sum_{i=1}^{n} i x_{i}^{2}$ & $30,100,200$ & {$[-10,10]$} & 0 \\
F3 & $f_{3}(x)=\sum_{i=1}^{n}\left|x_{i}\right|+\prod_{i=1}^{n}\left|x_{i}\right|$ & $30,100,200$ & {$[-10,10]$} & 0 \\
F4 & $f_{4}(x)=\sum_{i=1}^{n}\left(\sum_{j=1}^{i} x_{j}\right)^{2}$ & $30,100,200$ & {$[-100,100]$} & 0 \\
F5 & $f_{5}(x)=\max _{i}\left\{\left|x_{i}\right|, 1 \leq i \leq n\right\}$ & $30,100,200$ & {$[-100,100]$} & 0 \\
F6 & $f_{6}(x)=\sum_{i=1}^{n-1}\left[100\left(x_{i+1}-x_{i}^{2}\right)^{2}+\left(x_{i}-1\right)^{2}\right]$ & $30,100,200$ & {$[-30,30]$} & 0 \\
F7 & $f_{7}(x)=\sum_{i=1}^{n}\left(\left[x_{i}+0.5\right]\right)^{2}$ & $30,100,200$ & {$[-100,100]$} & 0 \\
F8 & $f_{8}(x)=\sum_{i=1}^{n} i x_{i}^{4}$ & $30,100,200$ & {$[-1.28,1.28]$} & 0 \\
F9 & $f_{9}(x)=\sum_{i=1}^{n} i x_{i}^{4}+\operatorname{random}[0,1)$ & $30,100,200$ & {$[-1.28,1.28]$} & 0 \\
F10 & $f_{10}(x)=\sum_{i=1}^{n}\left|x_{i}\right|^{i+1}$ & $30,100,200$ & {$[-1,1]$} & 0 \\
& & & & 0 \\
\hline
\end{tabular}




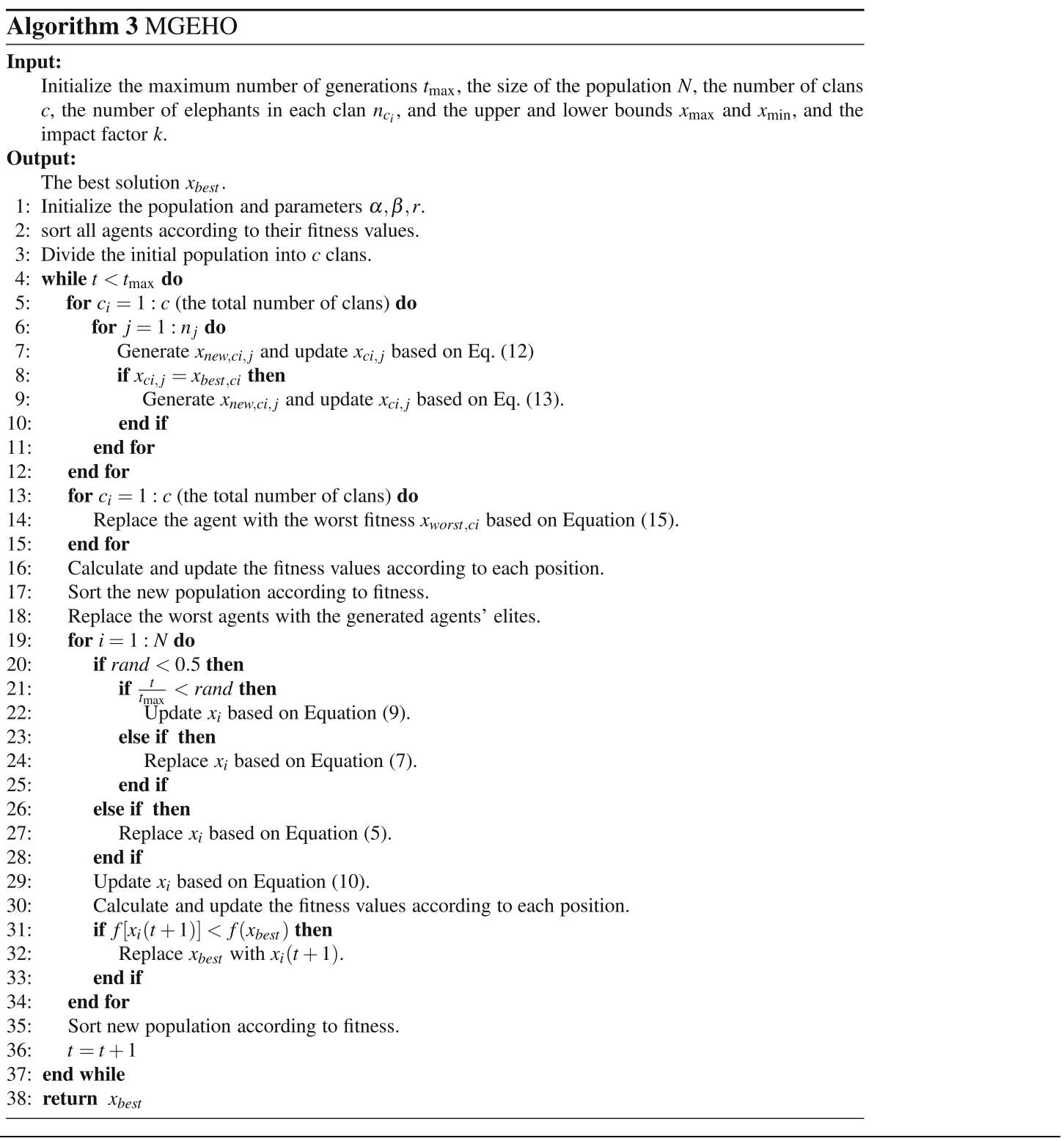

\subsection{Computational complexity of MGEHO}

One of the main measures of the optimization algorithm is the execution time. The modification of MGEHO consists of the following three main parts: mixture of MRFO's operators, adaptive inertia weights, and Gaussian variants. When the population size $N$, dimension of the optimization problem and maximum number of iterations are set to $N$, $D$ and $T$, respectively, the complexity of MGEHO can be calculated by the following way.
In MGEHO, the time used to initialize $N$ search agents is $O(N * D)$. The time to execute the clan-updating operator with adaptive inertia weights is $O(N * D)$. Next, the time to execute the separating operator with Gaussian variation is $O(N)$. Finally, the number of iterations is reduced because the MRFO operator is embedded within each iteration and no additional iterations are needed to be performed again. The time required to execute the MRFO operator is $O(N * D)$. When $\mathrm{T}$ iterations are performed, the total time complexity of MGEHO is $O(N * D+T(N * D+N+N *$ 
$D))=O(N *(D+T+2 * T * D))$, which is higher than the $O(T * N * D)$ of the basic EHO.

\section{Experimental results}

In this section, experimental results are presented. To verify the effectiveness and applicability of MGEHO, experiments are conducted on 33 classical benchmark functions, 32 CEC2014 and CEC2017 benchkmark functions. To ensure the fairness of the experiments, each algorithm is run 30 times independently on the benchmark functions to maintain stability. In addition, the population size $N$ for all algorithms is set to 30 , and the maximum number of iterations $t_{\max }$ is set to 500. Statistical tests are conducted to examine the differences between the different algorithms. The environmental conditions are an Intel (R) Core (TM) i5-9300H CPU @ $2.40 \mathrm{GHz}, 16 \mathrm{~GB}$ of RAM, and the Windows 10 operating system, and the simulation experiments are set up on the MATLAB R2019b platform.

\subsection{Tests on 33 benchmark functions}

\subsubsection{Benchmark functions}

In this subsection, 33 classical benchmark functions from reference [40] are selected, and the basic information regarding these functions is shown in Tables 1,2 and 3. Among them, F1-F10 are single-peaked functions that have only one global optimal solution in the global range and can test the convergence efficiency and exploration capability of each algorithm. F11-F23 are high-dimensional multipeaked functions that have multiple local extremes in the search space and are used to test the ability of each algorithm to search globally and jump out of local optima. F24-F33 are fixed-dimensional multipeak functions that are used to test the ability of each algorithm to maintain a balance between exploration and exploitation.

\subsubsection{Vaule analysis of the critical parameters}

The selection of the parameters can make an impact on the algorithm. $\omega_{l}, \omega_{u}$ and $k$ are three controlled parameters in MGEHO. In this subsection, value tests are performed to verify the effects of different parameters. During the designment of MGEHO, various values of these parameters are set for this experiment. It is worth noting that the interval of $\omega_{l}, \omega_{u}$ and $k$ is continuous and has numerous values. Here, $\left[\omega_{l}, \omega_{u}\right]$ is defined as $\{[0.1,0.7],[0.2,0.8],[0.3,0.9]\}$ and $k=1,2,3$. Since there are 3 values for each of the 2 sets of parameters, the fullorder factor will have $3 * 3=9$ design combinations. The 9 MGEHO variants are named after MGEHO1 to MGEHO9, and the settings of variants are shown in Table 4. Two functions, namely F5, F9, F11, F17, F29, F30, are employed from each category of unimodal, multimodal and fixed dimension multimodal functions The original EHO is selected for comparison with the other 9 different variants. Each algorithm is averaged over 30 independent runs of the fitness of the functions.

According to the results in Table 5, the different combinations of the proposed MGEHO outperform the original EHO, and the obtained superiority is proved by Friedman's test results and the associated $p$ values. Considering the overall ranking, the strongest contender is MGEHO3, followed by MGEHO2 and MGEHO6. In other words, MGEHO with $\left[\omega_{l}, \omega_{u}\right]=[0.1,0.7]$ and $k=3$ has the best performance among the nine combinations. Based on that, MGEHO with $\left[\omega_{l}, \omega_{u}\right]=[0.1,0.7]$ and $k=3$ is adopted for the subsequent experiments.

\subsubsection{Comparison with other algorithms}

MGEHO is compared with 9 other metaheuristics. These include EHO [19], enhanced EHO based on the $\gamma$ value (EEHO15) [22], OEHO [24], LFEHO [25], the IGWO [41], the EO [12], HHO [16], the WOA [15], and the SFO [14]. To make the experimental process fairer and more reliable, the parameters are set within each of the selected algorithms, as shown in Table 6. The other parameters are kept consistent except for those in Table 6.

\subsubsection{Scalability test for MGEHO}

Aiming to verify the adaptability of MGEHO under different conditions, the dimensions of F1-F23 are expanded from 30, 100 to 200, and the rest of the conditions are kept constant. After the experiments are completed, the mean value (Ave) and standard deviation $(S t d)$ are chosen as the evaluation indexes. The mean value reflects the accuracy and quality of a given algorithm, and the standard deviation reflects its stability. When solving a minimization problem, the smaller the mean value is, the better the performance of the algorithm. Similarity, the smaller the standard deviation is, the more stable the operation of the algorithm. The results are presented in Tables $7,8,9$, and 10 . In addition, the best solution in each table is shown in bold. 
Table 2 Details of the multimodal benchmark functions

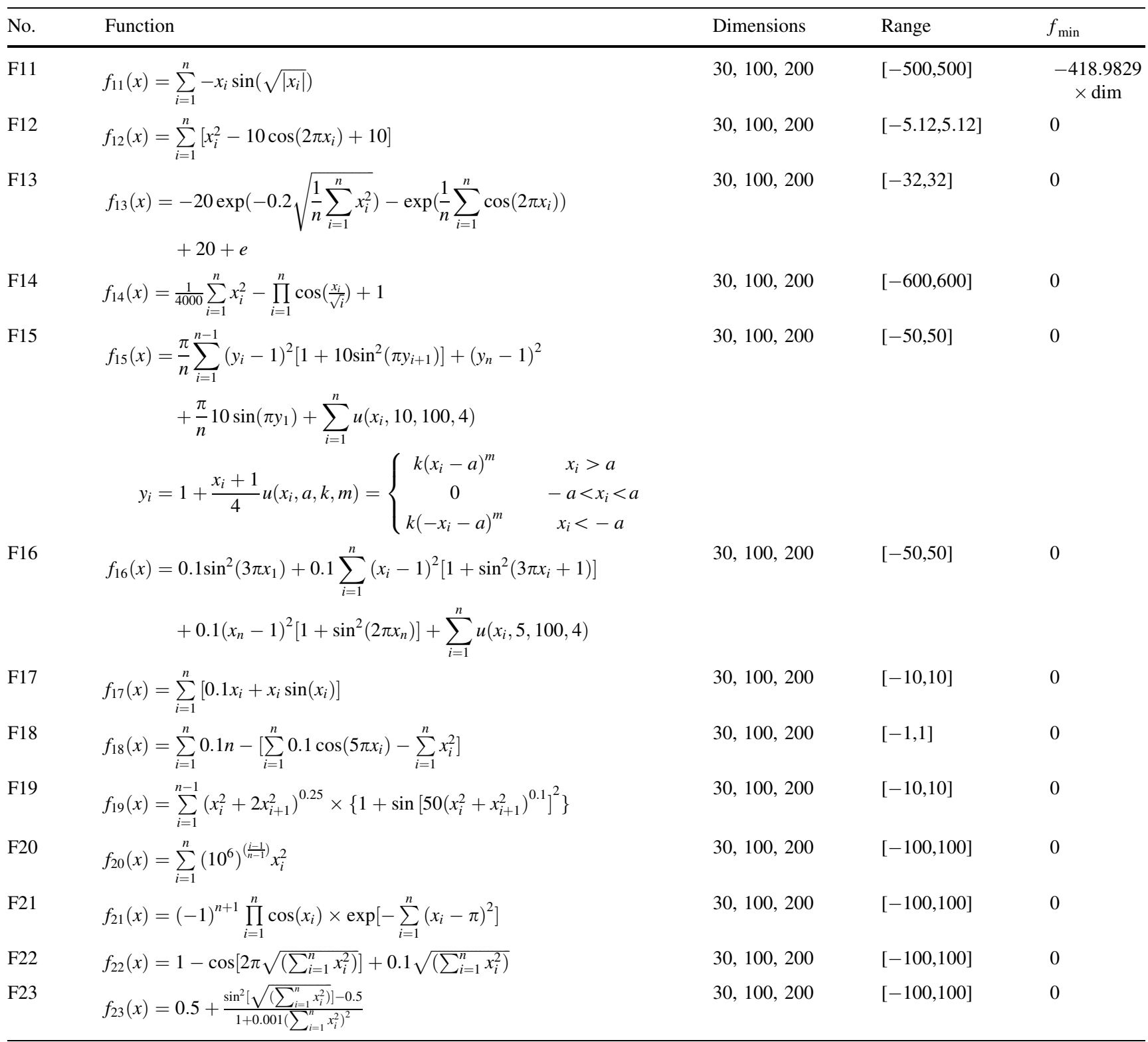

When the dimensionality of F1-F23 is 30 , the means and standard deviations recorded after 30 independent operations are shown in Table 7, where boldface indicates the relative optimal value of the corresponding comparison algorithm. It is clear that MGEHO performs better than the other approaches in general. In the single-peak function test, MGEHO obtains global optimal solutions on F1, F2, F4, F8, and F10 and the best solution among the 10 algorithms on F5 and F9 with the best performance stability. $\mathrm{HHO}$ achieves better solutions on F6, and the EO obtains a better solution on F7. This indicates that the exploratory nature of MGEHO is enhanced by incorporating somersault foraging from MRFO. Additionally, by combining the convergence factor and Gaussian perturbation, the population diversity is improved, enabling the algorithm to further develop the exploration space to obtain the best solutions. Regarding the multipeak functions, MGEHO achieves the global optimal solutions on F12, F14, F19, F20, F22, and F23 and outperforms the other algorithms on F13, F17, and F18. This indicates that MGEHO has a 
Table 3 Details of the fixed-dimension multimodal benchmark functions

\begin{tabular}{|c|c|c|c|c|}
\hline No. & Function & Dimensions & Range & $f_{\min }$ \\
\hline F24 & $f_{24}(x)=\left(\frac{1}{500}+\sum_{j=1}^{25} \frac{1}{j+\sum_{i=1}^{2}\left(x_{i}-a_{i j}\right)^{6}}\right)^{-1}$ & 2 & {$[-65,65]$} & 1 \\
\hline F25 & $f_{25}(x)=\sum_{i=1}^{11}\left[a_{i}-\frac{x_{1}\left(b_{i}^{2}+b_{i} x_{2}\right)}{b_{i}^{2}+b_{i} x_{3}+x_{4}}\right]^{2}$ & 4 & {$[-5,5]$} & 0.0003 \\
\hline F26 & $f_{26}(x)=4 x_{1}^{2}-2.1 x_{1}^{4}+\frac{1}{3} x_{1}^{6}+x_{1} x_{2}-4 x_{2}^{2}+4 x_{2}^{4}$ & 2 & {$[-5,5]$} & -1.0316 \\
\hline $\mathrm{F} 27$ & $f_{27}(x)=\left(x_{2}-\frac{5.1}{4 \pi^{2}} x_{1}^{2}+\frac{5}{\pi} x_{1}-6\right)^{2}+10\left(1-\frac{1}{8 \pi}\right) \cos x_{1}+10$ & 2 & {$[-5,5]$} & 0.398 \\
\hline F28 & $\begin{aligned} f_{28}(x) & =\left[1+\left(x_{1}+x_{2}+1\right)^{2}\left(19-14 x_{1}+3 x_{1}^{2}-14 x_{2}+6 x_{1} x_{2}+3 x_{2}^{2}\right)\right] \\
& \times\left[30+\left(2 x_{1}-3 x_{2}\right)^{2} \times\left(18-32 x_{1}+12 x_{1}^{2}+48 x_{2}-36 x_{1} x_{2}+27 x_{2}^{2}\right)\right]\end{aligned}$ & 2 & {$[-2,2]$} & 3 \\
\hline F29 & $f_{29}(x)=-\sum_{i=1}^{4} c_{i} \exp \left[-\sum_{j=1}^{3} a_{i j}\left(x_{j}-p_{i j}\right)^{2}\right]$ & 3 & {$[1,3]$} & -3.86 \\
\hline F30 & $f_{30}(x)=-\sum_{i=1}^{4} c_{i} \exp \left[-\sum_{j=1}^{6} a_{i j}\left(x_{j}-p_{i j}\right)^{2}\right]$ & 6 & {$[0,1]$} & -3.32 \\
\hline F31 & $f_{31}(x)=-\sum_{i=1}^{5}\left[\left(X-a_{i}\right)\left(X-a_{i}\right)^{T}+c_{i}\right]^{-1}$ & 4 & {$[0,10]$} & -10.1532 \\
\hline F32 & $f_{32}(x)=-\sum_{i=1}^{7}\left[\left(X-a_{i}\right)\left(X-a_{i}\right)^{T}+c_{i}\right]^{-1}$ & 4 & {$[0,10]$} & -10.4028 \\
\hline F33 & $f_{33}(x)=-\sum_{i=1}^{10}\left[\left(X-a_{i}\right)\left(X-a_{i}\right)^{T}+c_{i}\right]^{-1}$ & 4 & {$[0,10]$} & -10.5363 \\
\hline
\end{tabular}

Table 4 Combination of MGEHO's parameters

\begin{tabular}{|c|c|c|c|c|c|c|c|c|c|c|}
\hline \multirow[t]{2}{*}{ Parameters } & \multirow[t]{2}{*}{ Range } & \multicolumn{9}{|l|}{ Algorithms } \\
\hline & & MGEHO1 & MGEHO2 & MGEHO3 & MGEHO4 & MGEHO5 & MGEHO6 & MGEHO7 & MGEHO8 & MGEHO9 \\
\hline \multirow[t]{3}{*}[\omega_{l},\omega_{u}]{} & {$[0.1,0.7]$} & 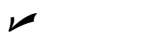 & レ & $レ$ & & & & & & \\
\hline & {$[0.2,0.8]$} & & & & レ & 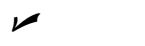 & 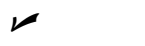 & & & \\
\hline & {$[0.3,0.9]$} & & & & & & & $\nu$ & $レ$ & $レ$ \\
\hline \multirow[t]{3}{*}{$k$} & 1 & 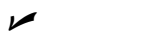 & & & $\nu$ & & & レ & & \\
\hline & 2 & & $\nu$ & & & & & & レ & \\
\hline & 3 & & & 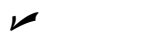 & & & レ & & & $\swarrow$ \\
\hline
\end{tabular}

strong ability to jump out of local optima; this stems from the clan updating operator and the convergence factor increasing the diversity and randomness of the population.

The experimental results on the fixed-dimension multimodal benchmark functions are shown in Table 8. Through the data shown in the table, MGEHO achieves superior solutions on F24, F26, F31, F32, F33 and the best average result on F30. The IGWO performs best on F27 and F28 and runs most stably on F29 and F30. HHO achieves the best result on F25, and the solution obtained on F29 is the closest to the global solution. This indicates that by introducing the nonlinear convergence factor, an algorithm can effectively control the balance between exploration and exploitation by maintaining diversification and intensification throughout the search process.
Table 9 shows the test results of different algorithms on the F1-F23 benchmark functions when the dimensionality is 100 . As the dimensionality increases, algorithms tend to need more information to search for optimal solutions; thus, increasing the dimensionality tests the ability of each algorithm to adapt to problems with different variants. The comparison results illustrate that the proposed MGEHO algorithm outperforms the other nine algorithms. Among the 23 benchmark functions, MGEHO surpasses the other algorithms on 17 of them. For F11, EEHO15 can perform better. LFEHO obtains the best solutions on F10 and F23, the IGWO has the best performance on F21, and the EO achieves the global optimal solutions on F12, F14, and F21. HHO has better results than those of the other algorithms on F6, F7, F12, F14, and F15. The SFO is able to provide 
Table 5 Comparison results of EHO with 9 variants of MGEHO

\begin{tabular}{|c|c|c|c|c|c|c|c|c|c|c|c|}
\hline \multicolumn{2}{|c|}{ Function } & \multirow{2}{*}{$\begin{array}{c}\text { EHO1 } \\
\begin{array}{c}1.17 E \\
-240\end{array}\end{array}$} & \multirow{2}{*}{$\begin{array}{c}\mathrm{EHO} 2 \\
\begin{array}{c}8.79 E \\
-308\end{array}\end{array}$} & \multirow{2}{*}{$\begin{array}{r}\text { EHO3 } \\
0.00 E \\
+00\end{array}$} & \multirow{2}{*}{$\begin{array}{c}\text { EHO4 } \\
\begin{array}{c}2.03 E \\
-191\end{array}\end{array}$} & \multirow{2}{*}{$\begin{array}{c}\text { EHO5 } \\
\begin{array}{c}6.53 E \\
-251\end{array}\end{array}$} & \multirow{2}{*}{$\begin{array}{c}\text { EHO6 } \\
\begin{array}{c}4.09 E \\
-279\end{array}\end{array}$} & \multirow{2}{*}{$\begin{array}{c}\text { EHO7 } \\
7.26 E \\
-156\end{array}$} & \multirow{2}{*}{$\begin{array}{c}\text { EHO8 } \\
\begin{array}{c}5.37 E \\
-206\end{array}\end{array}$} & \multirow{2}{*}{$\begin{array}{c}\text { EHO9 } \\
\begin{array}{c}1.27 E \\
-229\end{array}\end{array}$} & \multirow{2}{*}{$\begin{array}{r}\text { EHO } \\
\begin{array}{r}9.67 E \\
-04\end{array}\end{array}$} \\
\hline f1 & Avg & & & & & & & & & & \\
\hline & Std & $\begin{array}{r}0.00 E \\
+00\end{array}$ & $\begin{array}{r}0.00 E \\
+00\end{array}$ & $\begin{array}{r}0.00 E \\
+00\end{array}$ & $\begin{array}{r}0.00 E \\
+00\end{array}$ & $\begin{array}{r}0.00 E \\
+00\end{array}$ & $\begin{array}{r}0.00 E \\
+00\end{array}$ & $\begin{array}{l}3.40 E \\
-155\end{array}$ & $\begin{array}{r}0.00 E \\
+00\end{array}$ & $\begin{array}{r}0.00 E \\
+00\end{array}$ & $\begin{array}{r}7.89 E \\
-04\end{array}$ \\
\hline & Rank & 5 & 2 & 1 & 8 & 4 & 3 & 9 & 7 & 6 & 10 \\
\hline & $p$ value & $\begin{array}{r}1.21 E \\
-12\end{array}$ & $\begin{array}{r}6.58 E \\
-05\end{array}$ & $\mathrm{NaN}$ & $\begin{array}{r}1.21 E \\
-12\end{array}$ & $\begin{array}{r}1.21 E \\
-12\end{array}$ & $\begin{array}{r}1.21 E \\
-12\end{array}$ & $\begin{array}{r}1.21 E \\
-12\end{array}$ & $\begin{array}{r}1.21 E \\
-12\end{array}$ & $\begin{array}{r}1.21 E \\
-12\end{array}$ & $\begin{array}{r}1.21 E \\
-12\end{array}$ \\
\hline \multirow[t]{4}{*}{$\mathrm{f} 2$} & Avg & $\begin{array}{r}5.76 E \\
-05\end{array}$ & $\begin{array}{r}3.31 E \\
-05\end{array}$ & $\begin{array}{r}3.85 E \\
-05\end{array}$ & $\begin{array}{r}8.09 E \\
-05\end{array}$ & $\begin{array}{r}7.25 E \\
-05\end{array}$ & $\begin{array}{r}3.60 E \\
-05\end{array}$ & $\begin{array}{r}1.67 E \\
-04\end{array}$ & $\begin{array}{r}6.91 E \\
-05\end{array}$ & $\begin{array}{r}7.08 E \\
-05\end{array}$ & $\begin{array}{r}1.07 E \\
-03\end{array}$ \\
\hline & Std & $\begin{array}{r}4.58 E \\
-05\end{array}$ & $\begin{array}{r}3.02 E \\
-05\end{array}$ & $\begin{array}{r}2.78 E \\
-05\end{array}$ & $\begin{array}{r}4.92 E \\
-05\end{array}$ & $\begin{array}{r}5.57 E \\
-05\end{array}$ & $\begin{array}{r}3.24 E \\
-05\end{array}$ & $\begin{array}{r}1.94 E \\
-04\end{array}$ & $\begin{array}{r}5.52 E \\
-05\end{array}$ & $\begin{array}{r}5.75 E \\
-05\end{array}$ & $\begin{array}{r}1.52 E \\
-03\end{array}$ \\
\hline & Rank & 4 & 1 & 3 & 8 & 7 & 2 & 9 & 5 & 6 & 10 \\
\hline & $p$ value & $\begin{array}{r}2.92 E \\
-05\end{array}$ & $\mathrm{NaN}$ & $\begin{array}{r}2.12 E \\
-04\end{array}$ & $\begin{array}{r}7.66 E \\
-05\end{array}$ & $\begin{array}{r}1.37 E \\
-06\end{array}$ & $\begin{array}{r}5.69 E \\
-03\end{array}$ & $\begin{array}{r}2.13 E \\
-07\end{array}$ & $\begin{array}{r}3.03 E \\
-05\end{array}$ & $\begin{array}{r}4.07 E \\
-05\end{array}$ & $\begin{array}{r}8.48 E \\
-09\end{array}$ \\
\hline \multirow[t]{4}{*}{ f3 } & Avg & $\begin{array}{c}-1.24 E \\
+04\end{array}$ & $\begin{array}{c}-1.26 E \\
+04\end{array}$ & $\begin{array}{c}-1.26 E \\
+04\end{array}$ & $\begin{array}{c}-1.25 E \\
+04\end{array}$ & $\begin{array}{c}-1.26 E \\
+04\end{array}$ & $\begin{array}{c}-1.26 E \\
+04\end{array}$ & $\begin{array}{c}-1.25 E \\
+04\end{array}$ & $\begin{array}{c}-1.25 E \\
+04\end{array}$ & $\begin{array}{c}-1.24 E \\
+04\end{array}$ & $\begin{array}{c}-1.26 E \\
+04\end{array}$ \\
\hline & Std & $\begin{array}{r}1.39 E \\
-01\end{array}$ & $\begin{array}{r}4.08 E \\
-02\end{array}$ & $\begin{array}{r}8.54 E \\
-02\end{array}$ & $\begin{array}{r}5.34 E \\
-01\end{array}$ & $\begin{array}{r}2.96 E \\
-02\end{array}$ & $\begin{array}{r}1.63 E \\
-03\end{array}$ & $\begin{array}{r}1.37 E \\
-01\end{array}$ & $\begin{array}{r}2.16 E \\
+01\end{array}$ & $\begin{array}{r}7.24 E \\
-01\end{array}$ & $\begin{array}{r}7.41 E \\
+00\end{array}$ \\
\hline & Rank & 10 & 3 & 4 & 6 & 2 & 1 & 7 & 8 & 9 & 5 \\
\hline & $p$ value & $\begin{array}{r}3.02 E \\
-11\end{array}$ & $\begin{array}{r}3.02 E \\
-11\end{array}$ & $\begin{array}{r}3.02 E \\
-11\end{array}$ & $\begin{array}{r}3.02 E \\
-11\end{array}$ & $\begin{array}{r}8.12 E \\
-04\end{array}$ & $\mathrm{NaN}$ & $\begin{array}{r}3.02 E \\
-11\end{array}$ & $\begin{array}{r}3.02 E \\
-11\end{array}$ & $\begin{array}{r}3.02 E \\
-11\end{array}$ & $\begin{array}{r}2.71 E \\
-02\end{array}$ \\
\hline \multirow[t]{4}{*}{$\mathrm{f} 4$} & Avg & $\begin{array}{l}5.85 E \\
-248\end{array}$ & $\begin{array}{r}0.00 E \\
+00\end{array}$ & $\begin{array}{r}0.00 E \\
+00\end{array}$ & $\begin{array}{l}2.17 E \\
-204\end{array}$ & $\begin{array}{l}5.12 E \\
-240\end{array}$ & $\begin{array}{l}3.83 E \\
-289\end{array}$ & $\begin{array}{l}2.07 E \\
-131\end{array}$ & $\begin{array}{l}5.42 E \\
-205\end{array}$ & $\begin{array}{l}1.50 E \\
-226\end{array}$ & $\begin{array}{r}2.33 E \\
-04\end{array}$ \\
\hline & Std & $\begin{array}{r}0.00 E \\
+00\end{array}$ & $\begin{array}{r}0.00 E \\
+00\end{array}$ & $\begin{array}{r}0.00 E \\
+00\end{array}$ & $\begin{array}{r}0.00 E \\
+00\end{array}$ & $\begin{array}{r}0.00 E \\
+00\end{array}$ & $\begin{array}{r}0.00 E \\
+00\end{array}$ & $\begin{array}{l}1.14 E \\
-130\end{array}$ & $\begin{array}{r}0.00 E \\
+00\end{array}$ & $\begin{array}{r}0.00 E \\
+00\end{array}$ & $\begin{array}{r}3.12 E \\
-04\end{array}$ \\
\hline & Rank & 4 & 1 & 1 & 8 & 5 & 3 & 9 & 7 & 6 & 10 \\
\hline & $p$ value & $\begin{array}{r}1.21 E \\
-12\end{array}$ & $\mathrm{NaN}$ & $\mathrm{NaN}$ & $\begin{array}{r}1.21 E \\
-12\end{array}$ & $\begin{array}{r}1.21 E \\
-12\end{array}$ & $\begin{array}{r}1.21 E \\
-12\end{array}$ & $\begin{array}{r}1.21 E \\
-12\end{array}$ & $\begin{array}{r}1.21 E \\
-12\end{array}$ & $\begin{array}{r}1.21 E \\
-12\end{array}$ & $\begin{array}{r}1.21 E \\
-12\end{array}$ \\
\hline \multirow[t]{4}{*}{ f5 } & Avg & $\begin{array}{c}-3.86 E \\
+00\end{array}$ & $\begin{array}{c}-3.86 E \\
+00\end{array}$ & $\begin{array}{c}-3.86 E \\
+00\end{array}$ & $\begin{array}{c}-3.86 E \\
+00\end{array}$ & $\begin{array}{c}-3.86 E \\
+00\end{array}$ & $\begin{array}{c}-3.86 E \\
+00\end{array}$ & $\begin{array}{c}-3.86 E \\
+00\end{array}$ & $\begin{array}{c}-3.86 E \\
+00\end{array}$ & $\begin{array}{c}-3.86 E \\
+00\end{array}$ & $\begin{array}{c}-3.40 E \\
+00\end{array}$ \\
\hline & Std & $\begin{array}{r}8.45 E \\
-04\end{array}$ & $\begin{array}{r}5.35 E \\
-04\end{array}$ & $\begin{array}{r}1.06 E \\
-03\end{array}$ & $\begin{array}{r}2.68 E \\
-04\end{array}$ & $\begin{array}{r}1.35 E \\
-03\end{array}$ & $\begin{array}{r}1.12 E \\
-03\end{array}$ & $\begin{array}{r}6.38 E \\
-05\end{array}$ & $\begin{array}{r}8.79 E \\
-05\end{array}$ & $\begin{array}{r}3.15 E \\
-04\end{array}$ & $\begin{array}{r}3.83 E \\
-01\end{array}$ \\
\hline & Rank & 2 & 4 & 1 & 6 & 5 & 3 & 9 & 8 & 7 & 10 \\
\hline & $p$ value & $\begin{array}{r}1.33 E \\
-03\end{array}$ & $\begin{array}{r}1.81 E \\
-04\end{array}$ & $\mathrm{NaN}$ & $\begin{array}{r}5.19 E \\
-07\end{array}$ & $\begin{array}{r}7.96 E \\
-05\end{array}$ & $\begin{array}{r}5.94 E \\
-02\end{array}$ & $\begin{array}{r}1.33 E \\
-10\end{array}$ & $\begin{array}{r}3.82 E \\
-10\end{array}$ & $\begin{array}{r}1.16 E \\
-07\end{array}$ & $\begin{array}{r}3.02 E \\
-11\end{array}$ \\
\hline \multirow[t]{4}{*}{ f6 } & Avg & $\begin{array}{c}-3.26 E \\
+00\end{array}$ & $\begin{array}{c}-3.29 E \\
+00\end{array}$ & $\begin{array}{c}-3.31 E \\
+00\end{array}$ & $\begin{array}{c}-3.26 E \\
+00\end{array}$ & $\begin{array}{c}-3.26 E \\
+00\end{array}$ & $\begin{array}{c}-3.26 E \\
+00\end{array}$ & $\begin{array}{c}-3.25 E \\
+00\end{array}$ & $\begin{array}{c}-3.26 E \\
+00\end{array}$ & $\begin{array}{c}-3.26 E \\
+00\end{array}$ & $\begin{array}{c}-2.19 E \\
+00\end{array}$ \\
\hline & Std & $\begin{array}{r}6.85 E \\
-02\end{array}$ & $\begin{array}{r}5.93 E \\
-03\end{array}$ & $\begin{array}{r}3.40 E \\
-03\end{array}$ & $\begin{array}{r}6.96 E \\
-02\end{array}$ & $\begin{array}{r}6.76 E \\
-02\end{array}$ & $\begin{array}{r}6.31 E \\
-02\end{array}$ & $\begin{array}{r}6.69 E \\
-02\end{array}$ & $\begin{array}{r}6.23 E \\
-02\end{array}$ & $\begin{array}{r}6.19 E \\
-02\end{array}$ & $\begin{array}{r}4.15 E \\
-01\end{array}$ \\
\hline & Rank & 3 & 2 & 1 & 4 & 5 & 6 & 9 & 7 & 8 & 10 \\
\hline & $p$ value & $\begin{array}{r}7.73 E \\
-04\end{array}$ & $\begin{array}{r}3.51 E \\
-04\end{array}$ & $\mathrm{NaN}$ & $\begin{array}{r}9.33 E \\
-04\end{array}$ & $\begin{array}{r}8.42 E \\
-06\end{array}$ & $\begin{array}{r}4.38 E \\
-07\end{array}$ & $\begin{array}{r}7.06 E \\
-08\end{array}$ & $\begin{array}{r}1.76 E \\
-08\end{array}$ & $\begin{array}{r}2.01 E \\
-08\end{array}$ & $\begin{array}{r}3.02 E \\
-11\end{array}$ \\
\hline \multicolumn{2}{|c|}{ Average rank } & 4.6667 & 2.1667 & 1.8333 & 6.6667 & 4.6667 & 3.0000 & 8.6667 & 7.0000 & 7.0000 & 9.1667 \\
\hline \multicolumn{2}{|c|}{ Overall rank } & 4 & 2 & 1 & 6 & 4 & 3 & 9 & 7 & 7 & 10 \\
\hline
\end{tabular}

the best solution on F16, and the WOA has the best results on F12 and F21. In summary, MGEHO has certain reproducibility and stability advantages with respect to solving complex high-dimensional problems.
Table 10 shows the mean and standard deviation results obtained by different algorithms when the dimensionality of F1-F23 is increased to 200. The performances of the algorithms fluctuate differently as the dimensionality increases. Regarding the high-dimensional single-peaked 
Table 6 Parameter settings of different algorithms

\begin{tabular}{|c|c|c|}
\hline Algorithm & Parameter & Range \\
\hline \multirow[t]{4}{*}{ EHO } & $\alpha$ & 0.5 \\
\hline & $\beta$ & 0.2 \\
\hline & $c$ & 5 \\
\hline & $n_{c_{i}}$ & 6 \\
\hline \multirow[t]{5}{*}{ EEHO15 } & $\alpha$ & 0.5 \\
\hline & $\beta$ & 0.2 \\
\hline & $c$ & 5 \\
\hline & $n_{c_{i}}$ & 6 \\
\hline & $\gamma$ & 0.015 \\
\hline \multirow[t]{4}{*}{ OEHO } & $\alpha$ & 0.5 \\
\hline & $\beta$ & 0.2 \\
\hline & $N$ & 5 \\
\hline & $n_{j}$ & 6 \\
\hline \multirow[t]{5}{*}{ LFEHO } & $\alpha$ & 0.5 \\
\hline & $\beta_{1}$ & 0.1 \\
\hline & $\beta_{2}$ & 1.5 \\
\hline & $N$ & 5 \\
\hline & $n_{j}$ & 6 \\
\hline \multirow[t]{3}{*}{ IGWO } & $a$ & $(2,0)$ \\
\hline & $\vec{A}$ & {$[-a, a]$} \\
\hline & $\vec{C}$ & {$[0,2]$} \\
\hline \multirow[t]{3}{*}{ EO } & $a_{1}$ & 2 \\
\hline & $a_{2}$ & 1 \\
\hline & $G P$ & 0.5 \\
\hline \multirow[t]{2}{*}{ HHO } & $\beta$ & 1.5 \\
\hline & $E_{0}$ & {$[-1,1]$} \\
\hline \multirow[t]{3}{*}{ WOA } & $a$ & $(2,0)$ \\
\hline & $a_{2}$ & $(2,1)$ \\
\hline & $b$ & 1 \\
\hline \multirow[t]{4}{*}{ SFO } & $A$ & 4 \\
\hline & $e$ & 0.001 \\
\hline & Initial population of sailfish & 9 \\
\hline & Initial population of sardines & 21 \\
\hline \multirow[t]{6}{*}{ MGEHO } & $\alpha$ & 0.5 \\
\hline & $c$ & 5 \\
\hline & $n_{c_{i}}$ & 6 \\
\hline & $\omega_{l}$ & 0.1 \\
\hline & $\omega_{u}$ & 0.7 \\
\hline & $k$ & 3 \\
\hline
\end{tabular}

problem, MGEHO finds the best solutions for eight of the ten benchmark functions. In the high-dimensional multipeaked problem, MGEHO outperforms the other algorithms on 9 of the 13 benchmark functions. Therefore, the robustness and accuracy of the original algorithm are improved by mixing MRFO with EHO. It can be concluded that MGEHO is still the best-performing one among the nine other algorithms.

\subsubsection{Convergence curve analysis}

Convergence curves can reflect the abilities and convergence speeds of algorithms when dealing with optimization problems. The curves obtained with 30 dimensions are shown in Fig. 3. From these plots, it can be observed that MGEHO achieves the best solutions, which are more accurate than those of any other algorithm on both the single-peak and multipeak functions, thereby exhibiting superior performance. The results on the fixed-size functions indicate that MGEHO also ranks among the top algorithms in terms of its ability to balance exploration and exploitation.

\subsubsection{Statistical analysis}

García et al. [42] pointed out that when evaluating the performances of metaheuristic algorithms, it is not sufficient to make comparisons based only on their mean and standard deviation values. At the same time, there are inevitable chance factors that affect the experimental results during the experimental process. To examine the differences between different algorithms, the nonparametric Wilcoxon signed-rank test [43] and Friedman test [44] at the 5\% significance level are invoked in the experiments.

The results of the Wilcoxon signed-rank test are shown in Table 11, where the symbols " $+/=/-$ " represent that MGEHO is better than, equal to, or worse than the currently compared algorithm. $p$ values less than 0.05 indicate that there are statistically significant differences in the results. In the table, NaN indicates that the algorithm achieved the theoretically best solution for the corresponding problem; therefore, the comparison is not meaningful. In addition, the bold values in the table indicate values greater than $5 \%$. According to the experimental results, for the comparison between MGEHO and EHO and its variants, there are no statistically significant differences between MGEHO and EHO on F7 and F24; between MGEHO and EEHO15 on F15 and F16; between MGEHO and OEHO on F15, F16, F24, and F31; and between MGEHO and LFEHO on F10, F30, and F31. According to the comparison with other metaheuristics, there are no statistically significant differences between MGEHO and the IGWO on F15; between MGEHO and the EO on F16 and F25; between MGEHO and HHO on F25; between MGEHO and the SFO on F15 and F27; and between MGEHO and the WOA on F14, F18, and F30. Overall, MGEHO is significantly different from most of the algorithms in terms of the obtained results. In addition, MGEHO outperforms the other algorithms on most of the 
Table 7 Comparison of results obtained on the F1-F23 benchmark functions $(d=30)$

\begin{tabular}{|c|c|c|c|c|c|c|c|c|c|c|c|}
\hline Function & & MGEHO & $\mathrm{EHO}$ & EEHO15 & OEHO & LFEHO & IGWO & $\mathrm{EO}$ & $\mathrm{HHO}$ & SFO & WOA \\
\hline \multirow[t]{2}{*}{ F1 } & Mean & $\begin{array}{r}0.00 E \\
+00\end{array}$ & $\begin{array}{r}2.98 E \\
-05\end{array}$ & $\begin{array}{r}5.08 E \\
-06\end{array}$ & $\begin{array}{r}8.07 E \\
-05\end{array}$ & $\begin{array}{r}3.18 E \\
-03\end{array}$ & $\begin{array}{r}1.93 E \\
-28\end{array}$ & $\begin{array}{r}9.09 E \\
-41\end{array}$ & $\begin{array}{r}4.30 E \\
-92\end{array}$ & $\begin{array}{r}7.74 E \\
-11\end{array}$ & $\begin{array}{r}3.62 E \\
-70\end{array}$ \\
\hline & Std & $\begin{array}{r}0.00 E \\
+00\end{array}$ & $\begin{array}{r}3.59 E \\
-05\end{array}$ & $\begin{array}{r}8.57 E \\
-06\end{array}$ & $\begin{array}{r}2.26 E \\
-04\end{array}$ & $\begin{array}{r}4.30 E \\
-03\end{array}$ & $\begin{array}{r}2.76 E \\
-28\end{array}$ & $\begin{array}{r}1.94 E \\
-40\end{array}$ & $\begin{array}{r}2.35 E \\
-91\end{array}$ & $\begin{array}{r}1.13 E \\
-10\end{array}$ & $\begin{array}{r}1.96 E \\
-69\end{array}$ \\
\hline \multirow[t]{2}{*}{$\mathrm{F} 2$} & Mean & $\begin{array}{r}0.00 E \\
+00\end{array}$ & $\begin{array}{r}1.58 E \\
-05\end{array}$ & $\begin{array}{r}1.13 E \\
-06\end{array}$ & $\begin{array}{r}7.76 E \\
-05\end{array}$ & $\begin{array}{r}8.79 E \\
-04\end{array}$ & $\begin{array}{r}3.57 E \\
-29\end{array}$ & $\begin{array}{r}7.29 E \\
-42\end{array}$ & $\begin{array}{r}5.28 E \\
-96\end{array}$ & $\begin{array}{r}2.25 E \\
-09\end{array}$ & $\begin{array}{r}1.55 E \\
-75\end{array}$ \\
\hline & Std & $\begin{array}{r}0.00 E \\
+00\end{array}$ & $\begin{array}{r}2.43 E \\
-05\end{array}$ & $\begin{array}{r}4.58 E \\
-06\end{array}$ & $\begin{array}{r}1.52 E \\
-04\end{array}$ & $\begin{array}{r}1.24 E \\
-03\end{array}$ & $\begin{array}{r}6.78 E \\
-29\end{array}$ & $\begin{array}{r}1.81 E \\
-41\end{array}$ & $\begin{array}{r}2.47 E \\
-95\end{array}$ & $\begin{array}{r}6.38 E \\
-09\end{array}$ & $\begin{array}{r}5.15 E \\
-75\end{array}$ \\
\hline \multirow[t]{2}{*}{ F3 } & Mean & $\begin{array}{c}6.68 \mathrm{E} \\
-294\end{array}$ & $\begin{array}{r}3.64 E \\
-03\end{array}$ & $\begin{array}{r}6.17 E \\
-04\end{array}$ & $\begin{array}{r}1.08 E \\
-02\end{array}$ & $\begin{array}{r}2.17 E \\
-02\end{array}$ & $\begin{array}{r}6.56 E \\
-18\end{array}$ & $\begin{array}{r}4.11 E \\
-24\end{array}$ & $\begin{array}{r}3.83 E \\
-49\end{array}$ & $\begin{array}{r}4.60 E \\
-05\end{array}$ & $\begin{array}{r}1.44 E \\
-49\end{array}$ \\
\hline & Std & $\begin{array}{r}0.00 E \\
+00\end{array}$ & $\begin{array}{r}3.55 E \\
-03\end{array}$ & $\begin{array}{r}6.15 E \\
-04\end{array}$ & $\begin{array}{r}1.52 E \\
-02\end{array}$ & $\begin{array}{r}2.13 E \\
-02\end{array}$ & $\begin{array}{r}3.90 E \\
-18\end{array}$ & $\begin{array}{r}4.65 E \\
-24\end{array}$ & $\begin{array}{r}2.08 E \\
-48\end{array}$ & $\begin{array}{r}4.15 E \\
-05\end{array}$ & $\begin{array}{r}7.71 E \\
-49\end{array}$ \\
\hline \multirow[t]{2}{*}{ F4 } & Mean & $\begin{array}{r}0.00 E \\
+00\end{array}$ & $\begin{array}{r}6.95 E \\
-02\end{array}$ & $\begin{array}{r}2.84 E \\
-03\end{array}$ & $\begin{array}{r}7.47 E \\
-02\end{array}$ & $\begin{array}{r}7.57 E \\
-01\end{array}$ & $\begin{array}{r}1.43 E \\
-03\end{array}$ & $\begin{array}{r}2.98 E \\
-09\end{array}$ & $\begin{array}{r}1.53 E \\
-70\end{array}$ & $\begin{array}{r}3.08 E \\
-08\end{array}$ & $\begin{array}{r}4.31 E \\
+04\end{array}$ \\
\hline & Std & $\begin{array}{r}0.00 E \\
+00\end{array}$ & $\begin{array}{r}2.47 E \\
-01\end{array}$ & $\begin{array}{r}4.53 E \\
-03\end{array}$ & $\begin{array}{r}2.91 E \\
-01\end{array}$ & $\begin{array}{r}2.82 E \\
+00\end{array}$ & $\begin{array}{r}3.22 E \\
-03\end{array}$ & $\begin{array}{r}6.43 E \\
-09\end{array}$ & $\begin{array}{r}8.27 E \\
-70\end{array}$ & $\begin{array}{r}6.37 E \\
-08\end{array}$ & $\begin{array}{r}1.20 E \\
+04\end{array}$ \\
\hline \multirow[t]{2}{*}{ F5 } & Mean & $\begin{array}{c}4.46 \mathrm{E} \\
-297\end{array}$ & $\begin{array}{r}2.19 E \\
-03\end{array}$ & $\begin{array}{r}3.14 E \\
-04\end{array}$ & $\begin{array}{r}1.20 E \\
-03\end{array}$ & $\begin{array}{r}1.27 E \\
-02\end{array}$ & $\begin{array}{r}1.95 E \\
-05\end{array}$ & $\begin{array}{r}4.23 E \\
-10\end{array}$ & $\begin{array}{r}7.45 E \\
-50\end{array}$ & $\begin{array}{r}1.47 E \\
-06\end{array}$ & $\begin{array}{r}5.23 E \\
+01\end{array}$ \\
\hline & Std & $\begin{array}{r}\mathbf{0 . 0 0 E} \\
+\mathbf{0 0}\end{array}$ & $\begin{array}{r}3.49 E \\
-03\end{array}$ & $\begin{array}{r}4.08 E \\
-04\end{array}$ & $\begin{array}{r}1.26 E \\
-03\end{array}$ & $\begin{array}{r}1.29 E \\
-02\end{array}$ & $\begin{array}{r}1.70 E \\
-05\end{array}$ & $\begin{array}{r}8.89 E \\
-10\end{array}$ & $\begin{array}{r}2.18 E \\
-49\end{array}$ & $\begin{array}{r}1.09 E \\
-06\end{array}$ & $\begin{array}{r}2.86 E \\
+01\end{array}$ \\
\hline \multirow[t]{2}{*}{ F6 } & Mean & $\begin{array}{r}1.19 E \\
+00\end{array}$ & $\begin{array}{r}1.45 E \\
-01\end{array}$ & $\begin{array}{r}3.23 E \\
-02\end{array}$ & $\begin{array}{r}1.23 E \\
-01\end{array}$ & $\begin{array}{r}6.50 E \\
+00\end{array}$ & $\begin{array}{r}2.43 E \\
+01\end{array}$ & $\begin{array}{r}2.54 E \\
+01\end{array}$ & $\begin{array}{r}9.80 \mathrm{E} \\
-03\end{array}$ & $\begin{array}{r}3.55 E \\
-02\end{array}$ & $\begin{array}{r}2.81 E \\
+01\end{array}$ \\
\hline & Std & $\begin{array}{r}4.95 E \\
+00\end{array}$ & $\begin{array}{r}1.96 E \\
-01\end{array}$ & $\begin{array}{r}3.11 E \\
-02\end{array}$ & $\begin{array}{r}1.72 E \\
-01\end{array}$ & $\begin{array}{r}9.93 E \\
+00\end{array}$ & $\begin{array}{r}4.73 E \\
-01\end{array}$ & $\begin{array}{r}2.13 E \\
-01\end{array}$ & $\begin{array}{r}1.52 \mathrm{E} \\
-02\end{array}$ & $\begin{array}{r}3.45 E \\
-02\end{array}$ & $\begin{array}{r}4.99 E \\
-01\end{array}$ \\
\hline \multirow[t]{2}{*}{ F7 } & Mean & $\begin{array}{r}3.67 E \\
-03\end{array}$ & $\begin{array}{r}1.58 E \\
-03\end{array}$ & $\begin{array}{r}4.14 E \\
-04\end{array}$ & $\begin{array}{r}1.10 E \\
-03\end{array}$ & $\begin{array}{r}1.85 E \\
-01\end{array}$ & $\begin{array}{r}2.44 E \\
-02\end{array}$ & $\begin{array}{r}1.03 E \\
-05\end{array}$ & $\begin{array}{r}1.59 E \\
-04\end{array}$ & $\begin{array}{r}2.89 E \\
-02\end{array}$ & $\begin{array}{r}3.55 E \\
-01\end{array}$ \\
\hline & Std & $\begin{array}{r}7.03 E \\
-03\end{array}$ & $\begin{array}{r}2.91 E \\
-03\end{array}$ & $\begin{array}{r}5.54 E \\
-04\end{array}$ & $\begin{array}{r}1.97 E \\
-03\end{array}$ & $\begin{array}{r}2.04 E \\
-01\end{array}$ & $\begin{array}{r}7.42 E \\
-02\end{array}$ & $\begin{array}{r}9.38 \mathrm{E} \\
-06\end{array}$ & $\begin{array}{r}2.87 E \\
-04\end{array}$ & $\begin{array}{r}9.06 E \\
-02\end{array}$ & $\begin{array}{r}2.08 E \\
-01\end{array}$ \\
\hline \multirow[t]{2}{*}{ F8 } & Mean & $\begin{array}{r}0.00 E \\
+00\end{array}$ & $\begin{array}{r}1.31 E \\
-14\end{array}$ & $\begin{array}{r}7.21 E \\
-17\end{array}$ & $\begin{array}{r}1.59 E \\
-11\end{array}$ & $\begin{array}{r}3.07 E \\
-11\end{array}$ & $\begin{array}{r}3.59 E \\
-50\end{array}$ & $\begin{array}{r}3.54 E \\
-73\end{array}$ & $\begin{array}{c}1.17 E \\
-192\end{array}$ & $\begin{array}{r}1.97 E \\
-20\end{array}$ & $\begin{array}{l}7.38 E \\
-107\end{array}$ \\
\hline & Std & $\begin{array}{r}0.00 E \\
+00\end{array}$ & $\begin{array}{r}6.21 E \\
-14\end{array}$ & $\begin{array}{r}3.57 E \\
-16\end{array}$ & $\begin{array}{r}6.92 E \\
-11\end{array}$ & $\begin{array}{r}7.48 E \\
-11\end{array}$ & $\begin{array}{r}1.46 E \\
-49\end{array}$ & $\begin{array}{r}1.15 E \\
-72\end{array}$ & $\begin{array}{r}0.00 E \\
+00\end{array}$ & $\begin{array}{r}5.42 E \\
-20\end{array}$ & $\begin{array}{l}4.04 E \\
-106\end{array}$ \\
\hline \multirow[t]{2}{*}{ F9 } & Mean & $\begin{array}{r}7.01 E \\
-05\end{array}$ & $\begin{array}{r}1.34 E \\
-03\end{array}$ & $\begin{array}{r}1.97 E \\
-03\end{array}$ & $\begin{array}{r}1.44 E \\
-03\end{array}$ & $\begin{array}{r}4.47 E \\
-03\end{array}$ & $\begin{array}{r}2.73 E \\
-03\end{array}$ & $\begin{array}{r}1.05 E \\
-03\end{array}$ & $\begin{array}{r}1.77 E \\
-04\end{array}$ & $\begin{array}{r}5.05 E \\
-04\end{array}$ & $\begin{array}{r}4.06 E \\
-03\end{array}$ \\
\hline & Std & $\begin{array}{r}5.65 E \\
-05\end{array}$ & $\begin{array}{r}1.86 E \\
-03\end{array}$ & $\begin{array}{r}1.56 E \\
-03\end{array}$ & $\begin{array}{r}2.27 E \\
-03\end{array}$ & $\begin{array}{r}6.81 E \\
-03\end{array}$ & $\begin{array}{r}1.09 E \\
-03\end{array}$ & $\begin{array}{r}5.60 E \\
-04\end{array}$ & $\begin{array}{r}2.33 E \\
-04\end{array}$ & $\begin{array}{r}4.36 E \\
-04\end{array}$ & $\begin{array}{r}4.50 E \\
-03\end{array}$ \\
\hline \multirow[t]{2}{*}{ F10 } & Mean & $\begin{array}{r}0.00 E \\
+00\end{array}$ & $\begin{array}{r}1.06 E \\
-09\end{array}$ & $\begin{array}{r}1.03 E \\
-11\end{array}$ & $\begin{array}{r}9.92 E \\
-08\end{array}$ & $\begin{array}{r}3.33 E \\
-09\end{array}$ & $\begin{array}{r}1.54 E \\
-91\end{array}$ & $\begin{array}{l}3.77 E \\
-131\end{array}$ & $\begin{array}{l}3.51 E \\
-124\end{array}$ & $\begin{array}{r}2.38 E \\
-13\end{array}$ & $\begin{array}{l}9.70 E \\
-105\end{array}$ \\
\hline & Std & $\begin{array}{r}0.00 E \\
+00\end{array}$ & $\begin{array}{r}2.98 E \\
-09\end{array}$ & $\begin{array}{r}2.54 E \\
-11\end{array}$ & $\begin{array}{r}1.08 E \\
-07\end{array}$ & $\begin{array}{r}6.06 E \\
-09\end{array}$ & $\begin{array}{r}5.93 E \\
-91\end{array}$ & $\begin{array}{l}2.06 E \\
-130\end{array}$ & $\begin{array}{c}1.92 E \\
-123\end{array}$ & $\begin{array}{r}5.23 E \\
-13\end{array}$ & $\begin{array}{l}5.24 E \\
-104\end{array}$ \\
\hline \multirow[t]{2}{*}{ F11 } & Mean & $\begin{array}{c}-1.26 E \\
+04\end{array}$ & $\begin{array}{c}-1.26 E \\
+04\end{array}$ & $\begin{array}{c}-1.26 \mathrm{E} \\
+04\end{array}$ & $\begin{array}{c}-1.26 E \\
+04\end{array}$ & $\begin{array}{c}-1.26 E \\
+04\end{array}$ & $\begin{array}{c}-8.19 E \\
+03\end{array}$ & $\begin{array}{c}-8.94 E \\
+03\end{array}$ & $\begin{array}{c}-1.25 E \\
+04\end{array}$ & $\begin{array}{c}-3.67 E \\
+03\end{array}$ & $\begin{array}{c}-1.07 E \\
+04\end{array}$ \\
\hline & Std & $\begin{array}{r}1.41 E \\
+01\end{array}$ & $\begin{array}{r}3.41 E \\
+00\end{array}$ & $\begin{array}{r}2.20 \mathrm{E} \\
-01\end{array}$ & $\begin{array}{r}8.40 E \\
+00\end{array}$ & $\begin{array}{r}6.85 E \\
+00\end{array}$ & $\begin{array}{r}1.56 E \\
+03\end{array}$ & $\begin{array}{r}6.36 E \\
+02\end{array}$ & $\begin{array}{r}2.79 E \\
+02\end{array}$ & $\begin{array}{r}2.96 E \\
+02\end{array}$ & $\begin{array}{r}1.88 E \\
+03\end{array}$ \\
\hline \multirow[t]{2}{*}{$\mathrm{F} 12$} & Mean & $\begin{array}{r}0.00 \mathrm{E} \\
+\mathbf{0 0}\end{array}$ & $\begin{array}{r}9.67 E \\
-05\end{array}$ & $\begin{array}{r}3.29 E \\
-06\end{array}$ & $\begin{array}{r}1.85 E \\
-03\end{array}$ & $\begin{array}{r}4.04 E \\
-03\end{array}$ & $\begin{array}{r}1.89 E \\
+01\end{array}$ & $\begin{array}{r}0.00 E \\
+00\end{array}$ & $\begin{array}{r}0.00 E \\
+00\end{array}$ & $\begin{array}{r}3.54 E \\
-07\end{array}$ & $\begin{array}{r}0.00 \mathrm{E} \\
+\mathbf{0 0}\end{array}$ \\
\hline & Std & $\begin{array}{r}\text { 0.00E } \\
+\mathbf{0 0}\end{array}$ & $\begin{array}{r}2.53 E \\
-04\end{array}$ & $\begin{array}{r}8.45 E \\
-06\end{array}$ & $\begin{array}{r}3.52 E \\
-03\end{array}$ & $\begin{array}{r}8.68 E \\
-03\end{array}$ & $\begin{array}{r}1.05 E \\
+01\end{array}$ & $\begin{array}{r}0.00 E \\
+00\end{array}$ & $\begin{array}{r}\text { 0.00E } \\
+\mathbf{0 0}\end{array}$ & $\begin{array}{r}1.18 E \\
-06\end{array}$ & $\begin{array}{r}\text { 0.00E } \\
+\mathbf{0 0}\end{array}$ \\
\hline \multirow[t]{2}{*}{ F13 } & Mean & $\begin{array}{r}8.88 E \\
-16\end{array}$ & $\begin{array}{r}2.76 E \\
-03\end{array}$ & $\begin{array}{r}4.71 E \\
-04\end{array}$ & $\begin{array}{r}2.85 E \\
-03\end{array}$ & $\begin{array}{r}1.11 E \\
-02\end{array}$ & $\begin{array}{r}6.12 E \\
-14\end{array}$ & $\begin{array}{r}8.23 E \\
-15\end{array}$ & $\begin{array}{r}8.88 E \\
-16\end{array}$ & $\begin{array}{r}4.79 E \\
-06\end{array}$ & $\begin{array}{r}4.09 E \\
-15\end{array}$ \\
\hline & Std & $\begin{array}{r}0.00 E \\
+00\end{array}$ & $\begin{array}{r}3.02 E \\
-03\end{array}$ & $\begin{array}{r}7.06 E \\
-04\end{array}$ & $\begin{array}{r}3.48 E \\
-03\end{array}$ & $\begin{array}{r}1.39 E \\
-02\end{array}$ & $\begin{array}{r}1.13 E \\
-14\end{array}$ & $\begin{array}{r}2.07 E \\
-15\end{array}$ & $\begin{array}{r}0.00 E \\
+00\end{array}$ & $\begin{array}{r}5.69 E \\
-06\end{array}$ & $\begin{array}{r}2.70 E \\
-15\end{array}$ \\
\hline
\end{tabular}


Table 7 (continued)

\begin{tabular}{|c|c|c|c|c|c|c|c|c|c|c|c|}
\hline Function & & MGEHO & EHO & EEHO15 & OEHO & LFEHO & IGWO & EO & $\mathrm{HHO}$ & SFO & WOA \\
\hline \multirow[t]{2}{*}{ F14 } & Mean & $\begin{array}{r}0.00 E \\
+00\end{array}$ & $\begin{array}{r}1.82 E \\
-04\end{array}$ & $\begin{array}{r}4.93 E \\
-06\end{array}$ & $\begin{array}{r}6.54 E \\
-05\end{array}$ & $\begin{array}{r}1.19 E \\
-02\end{array}$ & $\begin{array}{r}3.78 E \\
-03\end{array}$ & $\begin{array}{r}0.00 E \\
+00\end{array}$ & $\begin{array}{r}0.00 \mathrm{E} \\
+00\end{array}$ & $\begin{array}{r}4.84 E \\
-12\end{array}$ & $\begin{array}{r}1.39 E \\
-02\end{array}$ \\
\hline & Std & $\begin{array}{r}0.00 E \\
+00\end{array}$ & $\begin{array}{r}5.07 E \\
-04\end{array}$ & $\begin{array}{r}1.15 E \\
-05\end{array}$ & $\begin{array}{r}1.00 E \\
-04\end{array}$ & $\begin{array}{r}3.00 E \\
-02\end{array}$ & $\begin{array}{r}8.63 E \\
-03\end{array}$ & $\begin{array}{r}0.00 E \\
+00\end{array}$ & $\begin{array}{r}\mathbf{0 . 0 0 E} \\
+00\end{array}$ & $\begin{array}{r}6.71 E \\
-12\end{array}$ & $\begin{array}{r}5.29 E \\
-02\end{array}$ \\
\hline \multirow[t]{2}{*}{ F15 } & Mean & $\begin{array}{r}9.02 E \\
-04\end{array}$ & $\begin{array}{r}2.62 E \\
-05\end{array}$ & $\begin{array}{r}2.07 E \\
-05\end{array}$ & $\begin{array}{r}3.82 E \\
-05\end{array}$ & $\begin{array}{r}3.37 E \\
-03\end{array}$ & $\begin{array}{r}2.52 E \\
-04\end{array}$ & $\begin{array}{r}5.45 \mathrm{E} \\
-07\end{array}$ & $\begin{array}{r}1.07 E \\
-05\end{array}$ & $\begin{array}{r}2.84 E \\
-02\end{array}$ & $\begin{array}{r}2.12 E \\
-02\end{array}$ \\
\hline & Std & $\begin{array}{r}3.29 E \\
-03\end{array}$ & $\begin{array}{r}4.00 E \\
-05\end{array}$ & $\begin{array}{r}2.45 E \\
-05\end{array}$ & $\begin{array}{r}5.59 E \\
-05\end{array}$ & $\begin{array}{r}6.19 E \\
-03\end{array}$ & $\begin{array}{r}1.17 E \\
-03\end{array}$ & $\begin{array}{r}7.30 \mathrm{E} \\
-07\end{array}$ & $\begin{array}{r}1.24 E \\
-05\end{array}$ & $\begin{array}{r}9.67 E \\
-02\end{array}$ & $\begin{array}{r}1.69 E \\
-02\end{array}$ \\
\hline \multirow[t]{2}{*}{ F16 } & Mean & $\begin{array}{r}2.12 E \\
-03\end{array}$ & $\begin{array}{r}9.73 E \\
-04\end{array}$ & $\begin{array}{r}3.10 E \\
-04\end{array}$ & $\begin{array}{r}9.44 E \\
-04\end{array}$ & $\begin{array}{r}3.03 E \\
-02\end{array}$ & $\begin{array}{r}1.30 E \\
-01\end{array}$ & $\begin{array}{r}2.53 E \\
-02\end{array}$ & $\begin{array}{r}9.31 E \\
-05\end{array}$ & $\begin{array}{r}7.92 E \\
-05\end{array}$ & $\begin{array}{r}4.76 E \\
-01\end{array}$ \\
\hline & Std & $\begin{array}{r}8.10 E \\
-03\end{array}$ & $\begin{array}{r}1.83 E \\
-03\end{array}$ & $\begin{array}{r}3.51 E \\
-04\end{array}$ & $\begin{array}{r}1.91 E \\
-03\end{array}$ & $\begin{array}{r}4.68 E \\
-02\end{array}$ & $\begin{array}{r}1.69 E \\
-01\end{array}$ & $\begin{array}{r}4.08 E \\
-02\end{array}$ & $\begin{array}{r}1.67 E \\
-04\end{array}$ & $\begin{array}{r}8.95 E \\
-05\end{array}$ & $\begin{array}{r}2.67 E \\
-01\end{array}$ \\
\hline \multirow[t]{2}{*}{ F17 } & Mean & $\begin{array}{l}1.11 E \\
-293\end{array}$ & $\begin{array}{r}3.02 E \\
-04\end{array}$ & $\begin{array}{r}6.37 E \\
-05\end{array}$ & $\begin{array}{r}5.33 E \\
-04\end{array}$ & $\begin{array}{r}2.49 E \\
-03\end{array}$ & $\begin{array}{r}3.25 E \\
-04\end{array}$ & $\begin{array}{r}1.38 E \\
-08\end{array}$ & $\begin{array}{r}5.06 E \\
-07\end{array}$ & $\begin{array}{r}4.10 E \\
-06\end{array}$ & $\begin{array}{r}1.72 E \\
-45\end{array}$ \\
\hline & Std & $\begin{array}{r}0.00 E \\
+00\end{array}$ & $\begin{array}{r}3.85 E \\
-04\end{array}$ & $\begin{array}{r}9.42 E \\
-05\end{array}$ & $\begin{array}{r}5.77 E \\
-04\end{array}$ & $\begin{array}{r}2.41 E \\
-03\end{array}$ & $\begin{array}{r}2.45 E \\
-04\end{array}$ & $\begin{array}{r}7.56 E \\
-08\end{array}$ & $\begin{array}{r}2.77 E \\
-06\end{array}$ & $\begin{array}{r}3.66 E \\
-06\end{array}$ & $\begin{array}{r}9.40 E \\
-45\end{array}$ \\
\hline \multirow[t]{2}{*}{ F18 } & Mean & $\begin{array}{c}-1.33 \mathrm{E} \\
-15\end{array}$ & $\begin{array}{r}1.09 E \\
-07\end{array}$ & $\begin{array}{r}5.01 E \\
-09\end{array}$ & $\begin{array}{r}8.01 E \\
-05\end{array}$ & $\begin{array}{r}1.79 E \\
-05\end{array}$ & $\begin{array}{c}-1.33 E \\
-15\end{array}$ & $\begin{array}{c}-1.33 E \\
-15\end{array}$ & $\begin{array}{c}-1.33 E \\
-15\end{array}$ & $\begin{array}{r}2.29 E \\
-09\end{array}$ & $\begin{array}{c}-1.32 E \\
-15\end{array}$ \\
\hline & Std & $\begin{array}{r}0.00 E \\
+00\end{array}$ & $\begin{array}{r}1.43 E \\
-07\end{array}$ & $\begin{array}{r}6.91 E \\
-09\end{array}$ & $\begin{array}{r}1.98 E \\
-04\end{array}$ & $\begin{array}{r}2.62 E \\
-05\end{array}$ & $\begin{array}{r}\text { 0.00E } \\
+00\end{array}$ & $\begin{array}{r}\text { 0.00E } \\
+\mathbf{0 0}\end{array}$ & $\begin{array}{r}\text { 0.00E } \\
+\mathbf{0 0}\end{array}$ & $\begin{array}{r}4.20 E \\
-09\end{array}$ & $\begin{array}{r}8.11 E \\
-17\end{array}$ \\
\hline \multirow[t]{2}{*}{ F19 } & Mean & $\begin{array}{r}\mathbf{0 . 0 0 E} \\
+\mathbf{0 0}\end{array}$ & $\begin{array}{r}9.02 E \\
-01\end{array}$ & $\begin{array}{r}2.16 E \\
-01\end{array}$ & $\begin{array}{r}9.90 E \\
-01\end{array}$ & $\begin{array}{r}1.48 E \\
+00\end{array}$ & $\begin{array}{r}6.25 E \\
-07\end{array}$ & $\begin{array}{r}2.01 E \\
-11\end{array}$ & $\begin{array}{r}7.83 E \\
-26\end{array}$ & $\begin{array}{r}9.51 E \\
-02\end{array}$ & $\begin{array}{r}4.12 E \\
-28\end{array}$ \\
\hline & Std & $\begin{array}{r}\mathbf{0 . 0 0 E} \\
+00\end{array}$ & $\begin{array}{r}4.91 E \\
-01\end{array}$ & $\begin{array}{r}1.30 E \\
-01\end{array}$ & $\begin{array}{r}6.23 E \\
-01\end{array}$ & $\begin{array}{r}8.79 E \\
-01\end{array}$ & $\begin{array}{r}5.43 E \\
-07\end{array}$ & $\begin{array}{r}1.36 E \\
-11\end{array}$ & $\begin{array}{r}3.50 E \\
-25\end{array}$ & $\begin{array}{r}5.73 E \\
-02\end{array}$ & $\begin{array}{r}2.06 E \\
-27\end{array}$ \\
\hline \multirow[t]{2}{*}{ F20 } & Mean & $\begin{array}{r}0.00 E \\
+00\end{array}$ & $\begin{array}{r}4.09 E \\
+00\end{array}$ & $\begin{array}{r}4.50 E \\
-01\end{array}$ & $\begin{array}{r}7.73 E \\
+00\end{array}$ & $\begin{array}{r}5.16 E \\
+02\end{array}$ & $\begin{array}{r}4.49 E \\
-25\end{array}$ & $\begin{array}{r}5.20 E \\
-37\end{array}$ & $\begin{array}{r}2.50 E \\
-91\end{array}$ & $\begin{array}{r}8.13 E \\
-06\end{array}$ & $\begin{array}{r}1.20 E \\
-68\end{array}$ \\
\hline & Std & $\begin{array}{r}0.00 E \\
+00\end{array}$ & $\begin{array}{r}6.95 E \\
+00\end{array}$ & $\begin{array}{r}1.24 E \\
+00\end{array}$ & $\begin{array}{r}2.64 E \\
+01\end{array}$ & $\begin{array}{r}1.05 E \\
+03\end{array}$ & $\begin{array}{r}1.16 E \\
-24\end{array}$ & $\begin{array}{r}1.88 E \\
-36\end{array}$ & $\begin{array}{r}1.36 E \\
-90\end{array}$ & $\begin{array}{r}1.34 E \\
-05\end{array}$ & $\begin{array}{r}6.40 E \\
-68\end{array}$ \\
\hline \multirow[t]{2}{*}{ F21 } & Mean & $\begin{array}{c}-9.87 E \\
-01\end{array}$ & $\begin{array}{c}-9.83 E \\
-01\end{array}$ & $\begin{array}{c}-9.95 E \\
-01\end{array}$ & $\begin{array}{c}-9.82 E \\
-01\end{array}$ & $\begin{array}{c}-7.72 E \\
-01\end{array}$ & $\begin{array}{r}\text { 0.00E } \\
+\mathbf{0 0}\end{array}$ & $\begin{array}{c}-3.33 E \\
-02\end{array}$ & $\begin{array}{c}-9.96 E \\
-01\end{array}$ & $\begin{array}{c}-9.42 E \\
-01\end{array}$ & $\begin{array}{r}\mathbf{0 . 0 0 E} \\
+\mathbf{0 0}\end{array}$ \\
\hline & Std & $\begin{array}{r}2.28 E \\
-02\end{array}$ & $\begin{array}{r}3.43 E \\
-02\end{array}$ & $\begin{array}{r}8.36 E \\
-03\end{array}$ & $\begin{array}{r}2.45 E \\
-02\end{array}$ & $\begin{array}{r}3.15 E \\
-01\end{array}$ & $\begin{array}{r}0.00 E \\
+00\end{array}$ & $\begin{array}{r}1.82 E \\
-01\end{array}$ & $\begin{array}{r}5.04 E \\
-03\end{array}$ & $\begin{array}{r}4.23 E \\
-02\end{array}$ & $\begin{array}{r}\mathbf{0 . 0 0 E} \\
+00\end{array}$ \\
\hline \multirow[t]{2}{*}{ F22 } & Mean & $\begin{array}{r}\mathbf{0 . 0 0 E} \\
+\mathbf{0 0}\end{array}$ & $\begin{array}{r}8.13 E \\
-03\end{array}$ & $\begin{array}{r}4.65 E \\
-04\end{array}$ & $\begin{array}{r}6.37 E \\
-03\end{array}$ & $\begin{array}{r}4.56 E \\
-02\end{array}$ & $\begin{array}{r}2.17 E \\
-01\end{array}$ & $\begin{array}{r}1.07 E \\
-01\end{array}$ & $\begin{array}{r}1.84 E \\
-47\end{array}$ & $\begin{array}{r}1.40 E \\
-03\end{array}$ & $\begin{array}{r}1.17 E \\
-01\end{array}$ \\
\hline & Std & $\begin{array}{r}\mathbf{0 . 0 0 E} \\
+\mathbf{0 0}\end{array}$ & $\begin{array}{r}7.31 E \\
-03\end{array}$ & $\begin{array}{r}1.18 E \\
-03\end{array}$ & $\begin{array}{r}1.85 E \\
-02\end{array}$ & $\begin{array}{r}5.42 E \\
-02\end{array}$ & $\begin{array}{r}3.79 E \\
-02\end{array}$ & $\begin{array}{r}2.54 E \\
-02\end{array}$ & $\begin{array}{r}1.00 E \\
-46\end{array}$ & $\begin{array}{r}1.76 E \\
-03\end{array}$ & $\begin{array}{r}7.46 E \\
-02\end{array}$ \\
\hline \multirow[t]{2}{*}{ F23 } & Mean & $\begin{array}{r}\mathbf{0 . 0 0 E} \\
+\mathbf{0 0}\end{array}$ & $\begin{array}{r}1.75 E \\
-03\end{array}$ & $\begin{array}{r}9.50 E \\
-06\end{array}$ & $\begin{array}{r}7.28 E \\
-04\end{array}$ & $\begin{array}{r}2.97 E \\
-02\end{array}$ & $\begin{array}{r}2.67 E \\
-01\end{array}$ & $\begin{array}{r}4.37 E \\
-02\end{array}$ & $\begin{array}{r}\text { 0.00E } \\
+00\end{array}$ & $\begin{array}{r}2.37 E \\
-07\end{array}$ & $\begin{array}{r}1.19 E \\
-01\end{array}$ \\
\hline & Std & $\begin{array}{r}\mathbf{0 . 0 0 E} \\
+\mathbf{0 0}\end{array}$ & $\begin{array}{r}2.67 E \\
-03\end{array}$ & $\begin{array}{r}2.32 E \\
-05\end{array}$ & $\begin{array}{r}8.97 E \\
-04\end{array}$ & $\begin{array}{r}2.66 E \\
-02\end{array}$ & $\begin{array}{r}8.89 E \\
-02\end{array}$ & $\begin{array}{r}4.00 E \\
-12\end{array}$ & $\begin{array}{r}\mathbf{0 . 0 0 E} \\
+\mathbf{0 0}\end{array}$ & $\begin{array}{r}5.81 E \\
-07\end{array}$ & $\begin{array}{r}1.21 E \\
-01\end{array}$ \\
\hline
\end{tabular}

benchmark functions. To make the results more convincing, the Friedman test at a significance level of $p=0.05$ is performed. The differences between the proposed MGEHO algorithm and other algorithms are tested by comparing the average rankings of the different algorithms on all benchmark functions; these rankings are shown in Tables 12 and 13. According to the literature, the algorithm with the lowest ranking is considered the most efficient algorithm. By comparison, MGEHO ranks first with different dimensions $(d=30,100,200)$, proving that the proposed algorithm has the largest competitive advantage.

\subsubsection{Diversity analysis of MGEHO}

To measure the performance of exploration and exploitation in metaheuristics, some researchers have tried indirect research methods, such as diversity analysis. In terms of the exploration and exploitation process, high diversity means that the solution is traversing and can spread across locations in the search space, thus allowing for extensive exploration. Reduced diversity then means that the agents search within a certain neighborhood and focuses on the 
Table 8 Comparison of results obtained on the F24-F33 benchmark functions

\begin{tabular}{|c|c|c|c|c|c|c|c|c|c|c|c|}
\hline Function & & MGEHO & $\mathrm{EHO}$ & EEHO15 & OEHO & LFEHO & IGWO & $\mathrm{EO}$ & $\mathrm{HHO}$ & SFO & WOA \\
\hline \multirow[t]{2}{*}{$\mathrm{F} 24$} & Mean & $\begin{array}{r}9.98 E \\
-01\end{array}$ & $\begin{array}{r}9.98 E \\
-01\end{array}$ & $\begin{array}{r}9.98 E \\
-01\end{array}$ & $\begin{array}{r}9.98 E \\
-01\end{array}$ & $\begin{array}{r}9.98 E \\
-01\end{array}$ & $\begin{array}{r}9.98 E \\
-01\end{array}$ & $\begin{array}{r}1.06 E \\
+00\end{array}$ & $\begin{array}{r}1.53 E \\
+00\end{array}$ & $\begin{array}{r}6.70 E \\
+00\end{array}$ & $\begin{array}{r}2.08 E \\
+00\end{array}$ \\
\hline & Std & $\begin{array}{r}1.10 E \\
-17\end{array}$ & $\begin{array}{r}8.88 E \\
-04\end{array}$ & $\begin{array}{r}2.20 E \\
-09\end{array}$ & $\begin{array}{r}1.27 E \\
-04\end{array}$ & $\begin{array}{r}2.95 E \\
-05\end{array}$ & $\begin{array}{r}1.13 E \\
-16\end{array}$ & $\begin{array}{r}3.62 E \\
-01\end{array}$ & $\begin{array}{r}1.29 E \\
+00\end{array}$ & $\begin{array}{r}3.31 E \\
+00\end{array}$ & $\begin{array}{r}2.59 E \\
+00\end{array}$ \\
\hline \multirow[t]{2}{*}{ F25 } & Mean & $\begin{array}{r}4.08 E \\
-04\end{array}$ & $\begin{array}{r}1.62 E \\
-03\end{array}$ & $\begin{array}{r}1.30 E \\
-03\end{array}$ & $\begin{array}{r}1.65 E \\
-03\end{array}$ & $\begin{array}{r}8.02 E \\
-04\end{array}$ & $\begin{array}{r}1.64 E \\
-03\end{array}$ & $\begin{array}{r}2.45 E \\
-03\end{array}$ & $\begin{array}{r}3.53 E \\
-04\end{array}$ & $\begin{array}{r}3.87 E \\
-04\end{array}$ & $\begin{array}{r}1.35 E \\
-03\end{array}$ \\
\hline & Std & $\begin{array}{r}2.28 E \\
-04\end{array}$ & $\begin{array}{r}1.82 E \\
-04\end{array}$ & $\begin{array}{r}5.16 E \\
-04\end{array}$ & $\begin{array}{r}1.35 E \\
-04\end{array}$ & $\begin{array}{r}5.48 E \\
-04\end{array}$ & $\begin{array}{r}5.09 E \\
-03\end{array}$ & $\begin{array}{r}6.08 E \\
-03\end{array}$ & $\begin{array}{r}4.31 E \\
-05\end{array}$ & $\begin{array}{r}9.72 E \\
-05\end{array}$ & $\begin{array}{r}3.40 E \\
-03\end{array}$ \\
\hline \multirow[t]{2}{*}{ F26 } & Mean & $\begin{array}{c}-1.03 E \\
+00\end{array}$ & $\begin{array}{c}-7.28 E \\
-01\end{array}$ & $\begin{array}{c}-1.03 E \\
+00\end{array}$ & $\begin{array}{c}-8.01 E \\
-01\end{array}$ & $\begin{array}{c}-1.02 E \\
+00\end{array}$ & $\begin{array}{c}-1.03 E \\
+00\end{array}$ & $\begin{array}{c}-1.03 E \\
+00\end{array}$ & $\begin{array}{c}-1.03 E \\
+00\end{array}$ & $\begin{array}{c}-1.02 E \\
+00\end{array}$ & $\begin{array}{c}-1.03 E \\
+00\end{array}$ \\
\hline & Std & $\begin{array}{r}1.07 E \\
-18\end{array}$ & $\begin{array}{r}3.69 E \\
-01\end{array}$ & $\begin{array}{r}1.95 E \\
-04\end{array}$ & $\begin{array}{r}3.78 E \\
-01\end{array}$ & $\begin{array}{r}8.12 E \\
-03\end{array}$ & $\begin{array}{r}6.18 E \\
-16\end{array}$ & $\begin{array}{r}6.52 E \\
-16\end{array}$ & $\begin{array}{r}1.35 E \\
-09\end{array}$ & $\begin{array}{r}1.84 E \\
-02\end{array}$ & $\begin{array}{r}9.89 E \\
-10\end{array}$ \\
\hline \multirow[t]{2}{*}{ F27 } & Mean & $\begin{array}{r}4.00 E \\
-01\end{array}$ & $\begin{array}{r}4.69 E \\
-01\end{array}$ & $\begin{array}{r}3.98 E \\
-01\end{array}$ & $\begin{array}{r}4.37 E \\
-01\end{array}$ & $\begin{array}{r}4.68 E \\
-01\end{array}$ & $\begin{array}{r}3.98 \mathrm{E} \\
-01\end{array}$ & $\begin{array}{r}3.98 E \\
-01\end{array}$ & $\begin{array}{r}3.98 E \\
-01\end{array}$ & $\begin{array}{r}3.99 E \\
-01\end{array}$ & $\begin{array}{r}3.98 E \\
-01\end{array}$ \\
\hline & Std & $\begin{array}{r}2.38 E \\
-03\end{array}$ & $\begin{array}{r}1.00 E \\
-01\end{array}$ & $\begin{array}{r}6.52 E \\
-04\end{array}$ & $\begin{array}{r}4.44 E \\
-02\end{array}$ & $\begin{array}{r}1.11 E \\
-01\end{array}$ & $\begin{array}{r}1.76 \mathrm{E} \\
-13\end{array}$ & $\begin{array}{r}0.00 E \\
+00\end{array}$ & $\begin{array}{r}1.47 E \\
-05\end{array}$ & $\begin{array}{r}8.03 E \\
-04\end{array}$ & $\begin{array}{r}2.49 E \\
-05\end{array}$ \\
\hline \multirow[t]{2}{*}{ F28 } & Mean & $\begin{array}{r}3.00 E \\
+00\end{array}$ & $\begin{array}{r}2.23 E \\
+01\end{array}$ & $\begin{array}{r}7.57 E \\
+00\end{array}$ & $\begin{array}{r}1.66 E \\
+01\end{array}$ & $\begin{array}{r}9.82 E \\
+00\end{array}$ & $\begin{array}{r}3.00 E \\
+00\end{array}$ & $\begin{array}{r}3.00 E \\
+00\end{array}$ & $\begin{array}{r}3.00 E \\
+00\end{array}$ & $\begin{array}{r}7.21 E \\
+00\end{array}$ & $\begin{array}{r}3.00 E \\
+00\end{array}$ \\
\hline & Std & $\begin{array}{r}1.97 E \\
-04\end{array}$ & $\begin{array}{r}1.05 E \\
+01\end{array}$ & $\begin{array}{r}1.04 E \\
+01\end{array}$ & $\begin{array}{r}1.04 E \\
+01\end{array}$ & $\begin{array}{r}9.89 E \\
+00\end{array}$ & $\begin{array}{r}1.45 \mathrm{E} \\
-15\end{array}$ & $\begin{array}{r}1.70 E \\
-15\end{array}$ & $\begin{array}{r}1.63 E \\
-07\end{array}$ & $\begin{array}{r}7.11 E \\
+00\end{array}$ & $\begin{array}{r}7.09 E \\
-05\end{array}$ \\
\hline \multirow[t]{2}{*}{ F29 } & Mean & $\begin{array}{c}-3.86 E \\
+00\end{array}$ & $\begin{array}{c}-3.28 E \\
+00\end{array}$ & $\begin{array}{c}-3.77 E \\
+00\end{array}$ & $\begin{array}{c}-3.58 E \\
+00\end{array}$ & $\begin{array}{c}-3.78 E \\
+00\end{array}$ & $\begin{array}{c}-3.86 E \\
+00\end{array}$ & $\begin{array}{c}-3.86 E \\
+00\end{array}$ & $\begin{array}{c}-3.86 \mathrm{E} \\
+00\end{array}$ & $\begin{array}{c}-3.83 E \\
+00\end{array}$ & $\begin{array}{c}-3.85 E \\
+00\end{array}$ \\
\hline & Std & $\begin{array}{r}3.46 E \\
-06\end{array}$ & $\begin{array}{r}4.32 E \\
-01\end{array}$ & $\begin{array}{r}8.66 E \\
-02\end{array}$ & $\begin{array}{r}2.00 E \\
-01\end{array}$ & $\begin{array}{r}1.24 E \\
-01\end{array}$ & $\begin{array}{r}2.52 \mathrm{E} \\
-15\end{array}$ & $\begin{array}{r}1.44 E \\
-03\end{array}$ & $\begin{array}{r}2.16 E \\
-03\end{array}$ & $\begin{array}{r}3.16 E \\
-02\end{array}$ & $\begin{array}{r}2.04 E \\
-02\end{array}$ \\
\hline \multirow[t]{2}{*}{ F30 } & Mean & $\begin{array}{c}-3.22 \mathrm{E} \\
+\mathbf{0 0}\end{array}$ & $\begin{array}{c}-2.04 E \\
+00\end{array}$ & $\begin{array}{c}-2.80 E \\
+00\end{array}$ & $\begin{array}{c}-2.13 E \\
+00\end{array}$ & $\begin{array}{c}-3.16 E \\
+00\end{array}$ & $\begin{array}{c}-3.30 E \\
+00\end{array}$ & $\begin{array}{c}-3.27 E \\
+00\end{array}$ & $\begin{array}{c}-3.09 E \\
+00\end{array}$ & $\begin{array}{l}-2.92 E \\
+00\end{array}$ & $\begin{array}{c}-3.22 E \\
+00\end{array}$ \\
\hline & Std & $\begin{array}{r}7.75 E \\
-02\end{array}$ & $\begin{array}{r}4.88 E \\
-01\end{array}$ & $\begin{array}{r}3.29 E \\
-01\end{array}$ & $\begin{array}{r}5.03 E \\
-01\end{array}$ & $\begin{array}{r}1.33 E \\
-01\end{array}$ & $\begin{array}{r}4.84 \mathrm{E} \\
-02\end{array}$ & $\begin{array}{r}6.05 E \\
-02\end{array}$ & $\begin{array}{r}1.23 E \\
-01\end{array}$ & $\begin{array}{r}2.22 E \\
-01\end{array}$ & $\begin{array}{r}1.28 E \\
-01\end{array}$ \\
\hline \multirow[t]{2}{*}{ F31 } & Mean & $\begin{array}{c}-1.02 E \\
+01\end{array}$ & $\begin{array}{c}-1.01 E \\
+01\end{array}$ & $\begin{array}{c}-1.02 E \\
+01\end{array}$ & $\begin{array}{c}-1.01 E \\
+01\end{array}$ & $\begin{array}{c}-1.01 E \\
+01\end{array}$ & $\begin{array}{c}-9.98 E \\
+00\end{array}$ & $\begin{array}{c}-8.47 E \\
+00\end{array}$ & $\begin{array}{c}-5.22 E \\
+00\end{array}$ & $\begin{array}{c}-1.00 E \\
+01\end{array}$ & $\begin{array}{l}-8.27 E \\
\quad+00\end{array}$ \\
\hline & Std & $\begin{array}{r}1.68 E \\
-05\end{array}$ & $\begin{array}{r}1.82 E \\
-02\end{array}$ & $\begin{array}{r}4.94 E \\
-04\end{array}$ & $\begin{array}{r}3.27 E \\
-02\end{array}$ & $\begin{array}{r}2.81 E \\
-02\end{array}$ & $\begin{array}{r}9.22 E \\
-01\end{array}$ & $\begin{array}{r}2.68 E \\
+00\end{array}$ & $\begin{array}{r}9.17 E \\
-01\end{array}$ & $\begin{array}{r}1.96 E \\
-01\end{array}$ & $\begin{array}{r}2.54 E \\
+00\end{array}$ \\
\hline \multirow[t]{2}{*}{ F32 } & Mean & $\begin{array}{c}-1.04 \mathrm{E} \\
+01\end{array}$ & $\begin{array}{c}-1.04 E \\
+01\end{array}$ & $\begin{array}{c}-1.04 E \\
+01\end{array}$ & $\begin{array}{c}-1.04 E \\
+01\end{array}$ & $\begin{array}{c}-1.04 E \\
+01\end{array}$ & $\begin{array}{c}-1.04 E \\
+01\end{array}$ & $\begin{array}{c}-9.65 E \\
+00\end{array}$ & $\begin{array}{c}-5.25 E \\
+00\end{array}$ & $\begin{array}{c}-1.03 E \\
+01\end{array}$ & $\begin{array}{c}-7.40 E \\
+00\end{array}$ \\
\hline & Std & $\begin{array}{r}7.30 \mathrm{E} \\
-06\end{array}$ & $\begin{array}{r}1.25 E \\
-02\end{array}$ & $\begin{array}{r}4.23 E \\
-04\end{array}$ & $\begin{array}{r}3.87 E \\
-02\end{array}$ & $\begin{array}{r}3.48 E \\
-02\end{array}$ & $\begin{array}{r}2.16 E \\
-05\end{array}$ & $\begin{array}{r}1.97 E \\
+00\end{array}$ & $\begin{array}{r}9.24 E \\
-01\end{array}$ & $\begin{array}{r}1.57 E \\
-01\end{array}$ & $\begin{array}{r}3.09 E \\
+00\end{array}$ \\
\hline \multirow[t]{2}{*}{ F33 } & Mean & $\begin{array}{c}-1.05 \mathrm{E} \\
+01\end{array}$ & $\begin{array}{c}-1.05 E \\
+01\end{array}$ & $\begin{array}{c}-1.05 E \\
+01\end{array}$ & $\begin{array}{c}-1.05 E \\
+01\end{array}$ & $\begin{array}{c}-1.05 E \\
+01\end{array}$ & $\begin{array}{c}-1.05 E \\
+01\end{array}$ & $\begin{array}{l}-9.10 E \\
+00\end{array}$ & $\begin{array}{c}-5.11 E \\
+00\end{array}$ & $\begin{array}{c}-1.04 E \\
+01\end{array}$ & $\begin{array}{c}-7.41 E \\
+00\end{array}$ \\
\hline & Std & $\begin{array}{r}7.57 E \\
-11\end{array}$ & $\begin{array}{r}2.16 E \\
-02\end{array}$ & $\begin{array}{r}4.40 E \\
-04\end{array}$ & $\begin{array}{r}5.27 E \\
-02\end{array}$ & $\begin{array}{r}4.87 E \\
-02\end{array}$ & $\begin{array}{r}4.93 E \\
-09\end{array}$ & $\begin{array}{r}2.69 E \\
+00\end{array}$ & $\begin{array}{r}1.09 E \\
+00\end{array}$ & $\begin{array}{r}1.37 E \\
-01\end{array}$ & $\begin{array}{r}3.24 E \\
+00\end{array}$ \\
\hline
\end{tabular}

process of exploitation. Diversity is calculated by the following equation:

$\operatorname{Div}_{j}=\frac{1}{n} \sum_{i=1}^{n}\left|\operatorname{median}\left(x^{j}\right)-x_{i}^{j}\right|$,

Div $=\frac{1}{m} \sum_{j=1}^{m} \operatorname{Div}_{j}$

where Div represents the overall diversity of the population, $\operatorname{Div}_{j}$ is the diversity of the data set consisting of the data in $j$-th dimension, $m$ is the dimension of the search agent, and $n$ is the population size, median $\left(x^{j}\right)$ stands for the median of the data in $j$-th dimension, and $x_{i}^{j}$ is the $j$-th dimension of $i$-th individual.

Figure 4 reflects the difference in diversity between the original EHO and MGEHO. The horizontal axis indicates the number of iterations and the vertical axis indicates the average distance between particles. It can be seen that both algorithms exhibit high diversity in the beginning phase due to the random initialization operation. Then, the 
Table 9 Comparison of results obtained on the F1-F23 benchmark functions $(d=100)$

\begin{tabular}{|c|c|c|c|c|c|c|c|c|c|c|c|}
\hline Function & & MGEHO & $\mathrm{EHO}$ & EEHO15 & OEHO & LFEHO & IGWO & EO & $\mathrm{HHO}$ & SFO & WOA \\
\hline \multirow[t]{2}{*}{ F1 } & Mean & $\begin{array}{r}0.00 E \\
+00\end{array}$ & $\begin{array}{r}2.35 E \\
-04\end{array}$ & $\begin{array}{r}1.69 E \\
-05\end{array}$ & $\begin{array}{r}2.90 E \\
-04\end{array}$ & $\begin{array}{r}1.24 E \\
-02\end{array}$ & $\begin{array}{r}2.19 E \\
-12\end{array}$ & $\begin{array}{r}5.27 E \\
-29\end{array}$ & $\begin{array}{r}7.66 E \\
-91\end{array}$ & $\begin{array}{r}2.82 E \\
-10\end{array}$ & $\begin{array}{r}5.66 E \\
-70\end{array}$ \\
\hline & Std & $\begin{array}{r}0.00 E \\
+00\end{array}$ & $\begin{array}{r}3.57 E \\
-04\end{array}$ & $\begin{array}{r}3.59 E \\
-05\end{array}$ & $\begin{array}{r}4.76 E \\
-04\end{array}$ & $\begin{array}{r}1.48 E \\
-02\end{array}$ & $\begin{array}{r}1.64 E \\
-12\end{array}$ & $\begin{array}{r}8.97 E \\
-29\end{array}$ & $\begin{array}{r}4.19 E \\
-90\end{array}$ & $\begin{array}{r}5.11 E \\
-10\end{array}$ & $\begin{array}{r}3.09 E \\
-69\end{array}$ \\
\hline \multirow[t]{2}{*}{$\mathrm{F} 2$} & Mean & $\begin{array}{r}0.00 E \\
+00\end{array}$ & $\begin{array}{r}2.56 E \\
-04\end{array}$ & $\begin{array}{r}3.51 E \\
-06\end{array}$ & $\begin{array}{r}8.64 E \\
-04\end{array}$ & $\begin{array}{r}5.23 E \\
-03\end{array}$ & $\begin{array}{r}8.79 E \\
-13\end{array}$ & $\begin{array}{r}1.31 E \\
-29\end{array}$ & $\begin{array}{r}6.63 E \\
-93\end{array}$ & $\begin{array}{r}1.14 E \\
-08\end{array}$ & $\begin{array}{r}2.40 E \\
-71\end{array}$ \\
\hline & Std & $\begin{array}{r}0.00 E \\
+00\end{array}$ & $\begin{array}{r}8.98 E \\
-04\end{array}$ & $\begin{array}{r}8.34 E \\
-06\end{array}$ & $\begin{array}{r}1.10 E \\
-03\end{array}$ & $\begin{array}{r}1.45 E \\
-02\end{array}$ & $\begin{array}{r}6.42 E \\
-13\end{array}$ & $\begin{array}{r}1.21 E \\
-29\end{array}$ & $\begin{array}{r}3.63 E \\
-92\end{array}$ & $\begin{array}{r}1.59 E \\
-08\end{array}$ & $\begin{array}{r}1.18 E \\
-70\end{array}$ \\
\hline \multirow[t]{2}{*}{ F3 } & Mean & $\begin{array}{c}4.21 \mathrm{E} \\
-296\end{array}$ & $\begin{array}{r}1.30 E \\
-02\end{array}$ & $\begin{array}{r}2.48 E \\
-03\end{array}$ & $\begin{array}{r}3.87 E \\
-02\end{array}$ & $\begin{array}{r}9.13 E \\
-02\end{array}$ & $\begin{array}{r}1.78 E \\
-08\end{array}$ & $\begin{array}{r}1.62 E \\
-17\end{array}$ & $\begin{array}{r}6.00 E \\
-51\end{array}$ & $\begin{array}{r}1.02 E \\
-04\end{array}$ & $\begin{array}{r}5.75 E \\
-49\end{array}$ \\
\hline & Std & $\begin{array}{r}\mathbf{0 . 0 0 E} \\
+00\end{array}$ & $\begin{array}{r}1.35 E \\
-02\end{array}$ & $\begin{array}{r}3.18 E \\
-03\end{array}$ & $\begin{array}{r}3.67 E \\
-02\end{array}$ & $\begin{array}{r}8.45 E \\
-02\end{array}$ & $\begin{array}{r}6.00 E \\
-09\end{array}$ & $\begin{array}{r}9.77 E \\
-18\end{array}$ & $\begin{array}{r}1.86 E \\
-50\end{array}$ & $\begin{array}{r}8.64 E \\
-05\end{array}$ & $\begin{array}{r}2.04 E \\
-48\end{array}$ \\
\hline \multirow[t]{2}{*}{ F4 } & Mean & $\begin{array}{r}0.00 E \\
+00\end{array}$ & $\begin{array}{r}1.19 E \\
+00\end{array}$ & $\begin{array}{r}7.87 E \\
-02\end{array}$ & $\begin{array}{r}1.89 E \\
+00\end{array}$ & $\begin{array}{r}1.65 E \\
+01\end{array}$ & $\begin{array}{r}5.58 E \\
+03\end{array}$ & $\begin{array}{r}5.28 E \\
+00\end{array}$ & $\begin{array}{r}3.07 E \\
-55\end{array}$ & $\begin{array}{r}7.45 E \\
-07\end{array}$ & $\begin{array}{r}1.11 E \\
+06\end{array}$ \\
\hline & Std & $\begin{array}{r}0.00 E \\
+00\end{array}$ & $\begin{array}{r}2.41 E \\
+00\end{array}$ & $\begin{array}{r}2.23 E \\
-01\end{array}$ & $\begin{array}{r}3.37 E \\
+00\end{array}$ & $\begin{array}{r}3.54 E \\
+01\end{array}$ & $\begin{array}{r}2.42 E \\
+03\end{array}$ & $\begin{array}{r}1.59 E \\
+01\end{array}$ & $\begin{array}{r}1.68 E \\
-54\end{array}$ & $\begin{array}{r}1.02 E \\
-06\end{array}$ & $\begin{array}{r}3.45 E \\
+05\end{array}$ \\
\hline \multirow[t]{2}{*}{ F5 } & Mean & $\begin{array}{c}2.84 E \\
-297\end{array}$ & $\begin{array}{r}1.31 E \\
-03\end{array}$ & $\begin{array}{r}2.29 E \\
-04\end{array}$ & $\begin{array}{r}9.94 E \\
-04\end{array}$ & $\begin{array}{r}1.88 E \\
-02\end{array}$ & $\begin{array}{r}4.09 E \\
+00\end{array}$ & $\begin{array}{r}1.52 E \\
-03\end{array}$ & $\begin{array}{r}5.06 E \\
-48\end{array}$ & $\begin{array}{r}1.68 E \\
-06\end{array}$ & $\begin{array}{r}7.21 E \\
+01\end{array}$ \\
\hline & Std & $\begin{array}{r}0.00 E \\
+00\end{array}$ & $\begin{array}{r}1.68 E \\
-03\end{array}$ & $\begin{array}{r}3.39 E \\
-04\end{array}$ & $\begin{array}{r}1.12 E \\
-03\end{array}$ & $\begin{array}{r}1.89 E \\
-02\end{array}$ & $\begin{array}{r}2.35 E \\
+00\end{array}$ & $\begin{array}{r}2.25 E \\
-03\end{array}$ & $\begin{array}{r}2.44 E \\
-47\end{array}$ & $\begin{array}{r}1.57 E \\
-06\end{array}$ & $\begin{array}{r}2.63 E \\
+01\end{array}$ \\
\hline \multirow[t]{2}{*}{ F6 } & Mean & $\begin{array}{r}3.92 E \\
+00\end{array}$ & $\begin{array}{r}1.23 E \\
+00\end{array}$ & $\begin{array}{r}1.19 E \\
-01\end{array}$ & $\begin{array}{r}5.65 E \\
-01\end{array}$ & $\begin{array}{r}2.05 E \\
+01\end{array}$ & $\begin{array}{r}9.76 E \\
+01\end{array}$ & $\begin{array}{r}9.61 E \\
+01\end{array}$ & $\begin{array}{r}5.29 \mathrm{E} \\
-02\end{array}$ & $\begin{array}{r}1.01 E \\
-01\end{array}$ & $\begin{array}{r}9.81 E \\
\quad+01\end{array}$ \\
\hline & Std & $\begin{array}{r}7.34 E \\
+00\end{array}$ & $\begin{array}{r}4.19 E \\
+00\end{array}$ & $\begin{array}{r}1.42 E \\
-01\end{array}$ & $\begin{array}{r}1.14 E \\
+00\end{array}$ & $\begin{array}{r}3.63 E \\
+01\end{array}$ & $\begin{array}{r}1.14 E \\
+00\end{array}$ & $\begin{array}{r}7.88 E \\
-01\end{array}$ & $\begin{array}{r}6.91 E \\
-02\end{array}$ & $\begin{array}{r}1.26 E \\
-01\end{array}$ & $\begin{array}{r}2.44 E \\
-01\end{array}$ \\
\hline \multirow[t]{2}{*}{ F7 } & Mean & $\begin{array}{r}1.30 E \\
-01\end{array}$ & $\begin{array}{r}5.20 E \\
-03\end{array}$ & $\begin{array}{r}8.97 E \\
-04\end{array}$ & $\begin{array}{r}4.72 E \\
-03\end{array}$ & $\begin{array}{r}1.11 E \\
+00\end{array}$ & $\begin{array}{r}7.94 E \\
+00\end{array}$ & $\begin{array}{r}3.81 E \\
+00\end{array}$ & $\begin{array}{r}6.53 E \\
-04\end{array}$ & $\begin{array}{r}2.27 E \\
-01\end{array}$ & $\begin{array}{r}4.16 E \\
+00\end{array}$ \\
\hline & Std & $\begin{array}{r}6.86 E \\
-01\end{array}$ & $\begin{array}{r}9.39 E \\
-03\end{array}$ & $\begin{array}{r}1.25 E \\
-03\end{array}$ & $\begin{array}{r}1.48 E \\
-02\end{array}$ & $\begin{array}{r}1.98 E \\
+00\end{array}$ & $\begin{array}{r}8.86 E \\
-01\end{array}$ & $\begin{array}{r}5.74 E \\
-01\end{array}$ & $\begin{array}{r}1.13 E \\
-03\end{array}$ & $\begin{array}{r}7.90 E \\
-01\end{array}$ & $\begin{array}{r}1.50 E \\
+00\end{array}$ \\
\hline \multirow[t]{2}{*}{ F8 } & Mean & $\begin{array}{r}0.00 E \\
+00\end{array}$ & $\begin{array}{r}6.36 E \\
-14\end{array}$ & $\begin{array}{r}1.97 E \\
-17\end{array}$ & $\begin{array}{r}7.02 E \\
-10\end{array}$ & $\begin{array}{r}2.83 E \\
-11\end{array}$ & $\begin{array}{r}2.32 E \\
-22\end{array}$ & $\begin{array}{r}1.22 E \\
-50\end{array}$ & $\begin{array}{r}3.38 E \\
-195\end{array}$ & $\begin{array}{r}1.73 E \\
-18\end{array}$ & $\begin{array}{l}7.77 E \\
-108\end{array}$ \\
\hline & Std & $\begin{array}{r}0.00 E \\
+00\end{array}$ & $\begin{array}{r}3.30 E \\
-13\end{array}$ & $\begin{array}{r}6.67 E \\
-17\end{array}$ & $\begin{array}{r}2.62 E \\
-09\end{array}$ & $\begin{array}{r}9.63 E \\
-11\end{array}$ & $\begin{array}{r}3.98 E \\
-22\end{array}$ & $\begin{array}{r}2.45 E \\
-50\end{array}$ & $\begin{array}{r}0.00 E \\
+00\end{array}$ & $\begin{array}{r}7.94 E \\
-18\end{array}$ & $\begin{array}{l}4.06 E \\
-107\end{array}$ \\
\hline \multirow[t]{2}{*}{ F9 } & Mean & $\begin{array}{r}5.57 E \\
-05\end{array}$ & $\begin{array}{r}2.15 E \\
-03\end{array}$ & $\begin{array}{r}2.74 E \\
-03\end{array}$ & $\begin{array}{r}2.04 E \\
-03\end{array}$ & $\begin{array}{r}6.03 E \\
-03\end{array}$ & $\begin{array}{r}1.21 E \\
-02\end{array}$ & $\begin{array}{r}2.49 E \\
-03\end{array}$ & $\begin{array}{r}1.53 E \\
-04\end{array}$ & $\begin{array}{r}6.10 E \\
-04\end{array}$ & $\begin{array}{r}3.95 E \\
-03\end{array}$ \\
\hline & Std & $\begin{array}{r}4.85 \mathrm{E} \\
-05\end{array}$ & $\begin{array}{r}3.97 E \\
-03\end{array}$ & $\begin{array}{r}2.12 E \\
-03\end{array}$ & $\begin{array}{r}3.62 E \\
-03\end{array}$ & $\begin{array}{r}7.87 E \\
-03\end{array}$ & $\begin{array}{r}3.48 E \\
-03\end{array}$ & $\begin{array}{r}1.09 E \\
-03\end{array}$ & $\begin{array}{r}1.76 E \\
-04\end{array}$ & $\begin{array}{r}5.23 E \\
-04\end{array}$ & $\begin{array}{r}4.94 E \\
-03\end{array}$ \\
\hline \multirow[t]{2}{*}{ F10 } & Mean & $\begin{array}{r}0.00 E \\
+00\end{array}$ & $\begin{array}{r}9.31 E \\
-10\end{array}$ & $\begin{array}{r}6.97 E \\
-12\end{array}$ & $\begin{array}{r}1.96 E \\
-07\end{array}$ & $\begin{array}{r}4.72 \mathrm{E} \\
-09\end{array}$ & $\begin{array}{r}1.02 E \\
-60\end{array}$ & $\begin{array}{r}1.58 E \\
-128\end{array}$ & $\begin{array}{l}1.78 E \\
-122\end{array}$ & $\begin{array}{r}1.83 E \\
-13\end{array}$ & $\begin{array}{l}2.81 E \\
\quad-105\end{array}$ \\
\hline & Std & $\begin{array}{r}0.00 E \\
+00\end{array}$ & $\begin{array}{r}1.94 E \\
-09\end{array}$ & $\begin{array}{r}1.46 E \\
-11\end{array}$ & $\begin{array}{r}3.95 E \\
-07\end{array}$ & $\begin{array}{r}1.13 E \\
-08\end{array}$ & $\begin{array}{r}4.63 E \\
-60\end{array}$ & $\begin{array}{c}8.32 E \\
-128\end{array}$ & $\begin{array}{c}9.73 E \\
-122\end{array}$ & $\begin{array}{r}3.61 E \\
-13\end{array}$ & $\begin{array}{l}1.07 E \\
-104\end{array}$ \\
\hline \multirow[t]{2}{*}{ F11 } & Mean & $\begin{array}{c}-4.19 E \\
+04\end{array}$ & $\begin{array}{c}-4.19 E \\
+04\end{array}$ & $\begin{array}{c}-4.19 \mathrm{E} \\
+\mathbf{0 4}\end{array}$ & $\begin{array}{c}-4.19 E \\
+04\end{array}$ & $\begin{array}{c}-4.19 E \\
+04\end{array}$ & $\begin{array}{c}-1.56 E \\
+04\end{array}$ & $\begin{array}{c}-2.60 E \\
+04\end{array}$ & $\begin{array}{c}-4.19 E \\
+04\end{array}$ & $\begin{array}{c}-6.80 E \\
+03\end{array}$ & $\begin{array}{c}-3.33 E \\
+04\end{array}$ \\
\hline & Std & $\begin{array}{r}8.39 E \\
+01\end{array}$ & $\begin{array}{r}1.26 E \\
+01\end{array}$ & $\begin{array}{r}4.46 \mathrm{E} \\
-01\end{array}$ & $\begin{array}{r}1.48 E \\
+01\end{array}$ & $\begin{array}{r}1.58 E \\
+01\end{array}$ & $\begin{array}{r}6.91 E \\
+03\end{array}$ & $\begin{array}{r}1.36 E \\
+03\end{array}$ & $\begin{array}{r}5.45 E \\
+00\end{array}$ & $\begin{array}{r}6.38 E \\
+02\end{array}$ & $\begin{array}{r}5.16 E \\
+03\end{array}$ \\
\hline \multirow[t]{2}{*}{$\mathrm{F} 12$} & Mean & $\begin{array}{r}0.00 \mathrm{E} \\
+\mathbf{0 0}\end{array}$ & $\begin{array}{r}4.52 E \\
-04\end{array}$ & $\begin{array}{r}3.12 E \\
-05\end{array}$ & $\begin{array}{r}5.36 E \\
-03\end{array}$ & $\begin{array}{r}2.15 E \\
-02\end{array}$ & $\begin{array}{r}1.35 E \\
+02\end{array}$ & $\begin{array}{r}\text { 0.00E } \\
+\mathbf{0 0}\end{array}$ & $\begin{array}{r}0.00 E \\
+00\end{array}$ & $\begin{array}{r}1.29 E \\
-06\end{array}$ & $\begin{array}{r}0.00 E \\
+00\end{array}$ \\
\hline & Std & $\begin{array}{r}0.00 E \\
+00\end{array}$ & $\begin{array}{r}1.54 E \\
-03\end{array}$ & $\begin{array}{r}1.03 E \\
-04\end{array}$ & $\begin{array}{r}7.83 E \\
-03\end{array}$ & $\begin{array}{r}5.20 E \\
-02\end{array}$ & $\begin{array}{r}5.47 E \\
+01\end{array}$ & $\begin{array}{r}\mathbf{0 . 0 0 E} \\
+\mathbf{0 0}\end{array}$ & $\begin{array}{r}0.00 E \\
+00\end{array}$ & $\begin{array}{r}3.21 E \\
-06\end{array}$ & $\begin{array}{r}0.00 E \\
+00\end{array}$ \\
\hline \multirow[t]{2}{*}{$\mathrm{F} 13$} & Mean & $\begin{array}{r}8.88 E \\
-16\end{array}$ & $\begin{array}{r}2.00 E \\
-03\end{array}$ & $\begin{array}{r}3.06 E \\
-04\end{array}$ & $\begin{array}{r}3.00 E \\
-03\end{array}$ & $\begin{array}{r}8.88 E \\
-03\end{array}$ & $\begin{array}{r}1.88 E \\
-07\end{array}$ & $\begin{array}{r}3.51 E \\
-14\end{array}$ & $\begin{array}{r}8.88 E \\
-16\end{array}$ & $\begin{array}{r}5.09 E \\
-06\end{array}$ & $\begin{array}{r}4.09 E \\
-15\end{array}$ \\
\hline & Std & $\begin{array}{r}0.00 E \\
+00\end{array}$ & $\begin{array}{r}2.11 E \\
-03\end{array}$ & $\begin{array}{r}5.29 E \\
-04\end{array}$ & $\begin{array}{r}3.17 E \\
-03\end{array}$ & $\begin{array}{r}8.78 E \\
-03\end{array}$ & $\begin{array}{r}7.00 E \\
-08\end{array}$ & $\begin{array}{r}6.56 E \\
-15\end{array}$ & $\begin{array}{r}0.00 E \\
+00\end{array}$ & $\begin{array}{r}4.17 E \\
-06\end{array}$ & $\begin{array}{r}2.35 E \\
-15\end{array}$ \\
\hline
\end{tabular}


Table 9 (continued)

\begin{tabular}{|c|c|c|c|c|c|c|c|c|c|c|c|}
\hline Function & & MGEHO & EHO & EEHO15 & OEHO & LFEHO & IGWO & EO & HHO & SFO & WOA \\
\hline \multirow[t]{2}{*}{ F14 } & Mean & $\begin{array}{r}0.00 E \\
+00\end{array}$ & $\begin{array}{r}2.12 E \\
-04\end{array}$ & $\begin{array}{r}5.20 E \\
-05\end{array}$ & $\begin{array}{r}2.08 E \\
-04\end{array}$ & $\begin{array}{r}2.55 E \\
-02\end{array}$ & $\begin{array}{r}1.28 E \\
-03\end{array}$ & $\begin{array}{r}0.00 E \\
+00\end{array}$ & $\begin{array}{r}0.00 E \\
+00\end{array}$ & $\begin{array}{r}5.17 E \\
-12\end{array}$ & $\begin{array}{r}9.21 E \\
-03\end{array}$ \\
\hline & Std & $\begin{array}{r}0.00 E \\
+00\end{array}$ & $\begin{array}{r}3.23 E \\
-04\end{array}$ & $\begin{array}{r}1.85 E \\
-04\end{array}$ & $\begin{array}{r}5.61 E \\
-04\end{array}$ & $\begin{array}{r}4.32 E \\
-02\end{array}$ & $\begin{array}{r}4.03 E \\
-03\end{array}$ & $\begin{array}{r}0.00 E \\
+00\end{array}$ & $\begin{array}{r}\mathbf{0 . 0 0 E} \\
+00\end{array}$ & $\begin{array}{r}8.32 E \\
-12\end{array}$ & $\begin{array}{r}5.04 E \\
-02\end{array}$ \\
\hline \multirow[t]{2}{*}{ F15 } & Mean & $\begin{array}{r}1.57 E \\
-05\end{array}$ & $\begin{array}{r}3.12 E \\
-05\end{array}$ & $\begin{array}{r}1.18 E \\
-05\end{array}$ & $\begin{array}{r}1.04 E \\
-05\end{array}$ & $\begin{array}{r}2.03 E \\
-03\end{array}$ & $\begin{array}{r}2.04 E \\
-01\end{array}$ & $\begin{array}{r}3.78 E \\
-02\end{array}$ & $\begin{array}{r}4.47 \mathrm{E} \\
-06\end{array}$ & $\begin{array}{r}3.10 E \\
-03\end{array}$ & $\begin{array}{r}3.77 E \\
-02\end{array}$ \\
\hline & Std & $\begin{array}{r}1.96 E \\
-05\end{array}$ & $\begin{array}{r}6.72 E \\
-05\end{array}$ & $\begin{array}{r}1.13 E \\
-05\end{array}$ & $\begin{array}{r}1.21 E \\
-05\end{array}$ & $\begin{array}{r}4.45 E \\
-03\end{array}$ & $\begin{array}{r}6.42 E \\
-02\end{array}$ & $\begin{array}{r}1.13 E \\
-02\end{array}$ & $\begin{array}{r}5.97 E \\
-06\end{array}$ & $\begin{array}{r}7.98 E \\
-03\end{array}$ & $\begin{array}{r}1.84 E \\
-02\end{array}$ \\
\hline \multirow[t]{2}{*}{ F16 } & Mean & $\begin{array}{r}2.72 E \\
-03\end{array}$ & $\begin{array}{r}5.90 E \\
-04\end{array}$ & $\begin{array}{r}3.48 E \\
-04\end{array}$ & $\begin{array}{r}6.05 E \\
-04\end{array}$ & $\begin{array}{r}5.96 E \\
-02\end{array}$ & $\begin{array}{r}5.99 E \\
+00\end{array}$ & $\begin{array}{r}5.86 E \\
+00\end{array}$ & $\begin{array}{r}1.76 E \\
-04\end{array}$ & $\begin{array}{r}1.71 E \\
-04\end{array}$ & $\begin{array}{r}3.06 E \\
+00\end{array}$ \\
\hline & Std & $\begin{array}{r}4.44 E \\
-03\end{array}$ & $\begin{array}{r}9.10 E \\
-04\end{array}$ & $\begin{array}{r}3.53 E \\
-04\end{array}$ & $\begin{array}{r}9.08 E \\
-04\end{array}$ & $\begin{array}{r}9.26 E \\
-02\end{array}$ & $\begin{array}{r}6.21 E \\
-01\end{array}$ & $\begin{array}{r}1.15 E \\
+00\end{array}$ & $\begin{array}{r}2.47 E \\
-04\end{array}$ & $\begin{array}{r}1.76 E \\
-04\end{array}$ & $\begin{array}{r}9.92 E \\
-01\end{array}$ \\
\hline \multirow[t]{2}{*}{ F17 } & Mean & $\begin{array}{c}3.97 \mathbf{E} \\
-287\end{array}$ & $\begin{array}{r}8.17 E \\
-04\end{array}$ & $\begin{array}{r}3.65 E \\
-04\end{array}$ & $\begin{array}{r}1.58 E \\
-03\end{array}$ & $\begin{array}{r}8.09 E \\
-03\end{array}$ & $\begin{array}{r}4.92 E \\
-03\end{array}$ & $\begin{array}{r}4.64 E \\
-18\end{array}$ & $\begin{array}{r}6.80 E \\
-06\end{array}$ & $\begin{array}{r}1.38 E \\
-05\end{array}$ & $\begin{array}{r}1.05 E \\
-50\end{array}$ \\
\hline & Std & $\begin{array}{r}0.00 E \\
+00\end{array}$ & $\begin{array}{r}8.78 E \\
-04\end{array}$ & $\begin{array}{r}5.41 E \\
-04\end{array}$ & $\begin{array}{r}2.07 E \\
-03\end{array}$ & $\begin{array}{r}8.11 E \\
-03\end{array}$ & $\begin{array}{r}3.49 E \\
-03\end{array}$ & $\begin{array}{r}4.64 E \\
-18\end{array}$ & $\begin{array}{r}3.73 E \\
-05\end{array}$ & $\begin{array}{r}1.52 E \\
-05\end{array}$ & $\begin{array}{r}2.77 E \\
-50\end{array}$ \\
\hline \multirow[t]{2}{*}{ F18 } & Mean & $\begin{array}{r}1.95 \mathrm{E} \\
-14\end{array}$ & $\begin{array}{r}1.51 E \\
-06\end{array}$ & $\begin{array}{r}2.39 E \\
-08\end{array}$ & $\begin{array}{r}3.29 E \\
-04\end{array}$ & $\begin{array}{r}7.91 E \\
-05\end{array}$ & $\begin{array}{r}4.07 E \\
-14\end{array}$ & $\begin{array}{r}1.95 E \\
-14\end{array}$ & $\begin{array}{r}1.95 E \\
-14\end{array}$ & $\begin{array}{r}9.74 E \\
-09\end{array}$ & $\begin{array}{r}1.95 E \\
-14\end{array}$ \\
\hline & Std & $\begin{array}{r}0.00 E \\
+00\end{array}$ & $\begin{array}{r}5.00 E \\
-06\end{array}$ & $\begin{array}{r}4.11 E \\
-08\end{array}$ & $\begin{array}{r}1.30 E \\
-03\end{array}$ & $\begin{array}{r}2.55 E \\
-04\end{array}$ & $\begin{array}{r}6.10 E \\
-15\end{array}$ & $\begin{array}{r}\mathbf{0 . 0 0 E} \\
+\mathbf{0 0}\end{array}$ & $\begin{array}{r}\mathbf{0 . 0 0 E} \\
+\mathbf{0 0}\end{array}$ & $\begin{array}{r}2.67 E \\
-08\end{array}$ & $\begin{array}{r}0.00 E \\
+00\end{array}$ \\
\hline \multirow[t]{2}{*}{ F19 } & Mean & $\begin{array}{r}\mathbf{0 . 0 0 E} \\
+\mathbf{0 0}\end{array}$ & $\begin{array}{r}2.62 E \\
+00\end{array}$ & $\begin{array}{r}6.26 E \\
-01\end{array}$ & $\begin{array}{r}2.95 E \\
+00\end{array}$ & $\begin{array}{r}4.60 E \\
+00\end{array}$ & $\begin{array}{r}3.18 E \\
-01\end{array}$ & $\begin{array}{r}4.41 E \\
-08\end{array}$ & $\begin{array}{r}3.11 E \\
-25\end{array}$ & $\begin{array}{r}2.94 E \\
-01\end{array}$ & $\begin{array}{r}1.87 E \\
-28\end{array}$ \\
\hline & Std & $\begin{array}{r}\mathbf{0 . 0 0 E} \\
+00\end{array}$ & $\begin{array}{r}1.19 E \\
+00\end{array}$ & $\begin{array}{r}2.77 E \\
-01\end{array}$ & $\begin{array}{r}1.52 E \\
+00\end{array}$ & $\begin{array}{r}2.58 E \\
+00\end{array}$ & $\begin{array}{r}1.28 E \\
-01\end{array}$ & $\begin{array}{r}1.50 E \\
-08\end{array}$ & $\begin{array}{r}1.23 E \\
-24\end{array}$ & $\begin{array}{r}1.86 E \\
-01\end{array}$ & $\begin{array}{r}7.87 E \\
-28\end{array}$ \\
\hline \multirow[t]{2}{*}{ F20 } & Mean & $\begin{array}{r}0.00 E \\
+00\end{array}$ & $\begin{array}{r}1.28 E \\
+01\end{array}$ & $\begin{array}{r}3.21 E \\
+00\end{array}$ & $\begin{array}{r}2.66 E \\
+01\end{array}$ & $\begin{array}{r}1.30 E \\
+03\end{array}$ & $\begin{array}{r}3.77 E \\
-09\end{array}$ & $\begin{array}{r}1.34 E \\
-25\end{array}$ & $\begin{array}{r}9.03 E \\
-84\end{array}$ & $\begin{array}{r}5.33 E \\
-05\end{array}$ & $\begin{array}{r}8.69 E \\
-68\end{array}$ \\
\hline & Std & $\begin{array}{r}\mathbf{0 . 0 0 E} \\
+00\end{array}$ & $\begin{array}{r}2.23 E \\
+01\end{array}$ & $\begin{array}{r}8.32 E \\
+00\end{array}$ & $\begin{array}{r}4.29 E \\
+01\end{array}$ & $\begin{array}{r}2.13 E \\
+03\end{array}$ & $\begin{array}{r}4.31 E \\
-09\end{array}$ & $\begin{array}{r}2.05 E \\
-25\end{array}$ & $\begin{array}{r}4.95 E \\
-83\end{array}$ & $\begin{array}{r}1.52 E \\
-04\end{array}$ & $\begin{array}{r}2.69 E \\
-67\end{array}$ \\
\hline \multirow[t]{2}{*}{ F21 } & Mean & $\begin{array}{c}-9.61 E \\
-01\end{array}$ & $\begin{array}{c}-9.17 E \\
-01\end{array}$ & $\begin{array}{c}-1.00 E \\
+00\end{array}$ & $\begin{array}{c}-9.51 E \\
-01\end{array}$ & $\begin{array}{c}-5.54 E \\
-01\end{array}$ & $\begin{array}{r}\mathbf{0 . 0 0 E} \\
+\mathbf{0 0}\end{array}$ & $\begin{array}{r}\mathbf{0 . 0 0 E} \\
+\mathbf{0 0}\end{array}$ & $\begin{array}{c}-9.67 E \\
-01\end{array}$ & $\begin{array}{c}-8.77 E \\
-01\end{array}$ & $\begin{array}{r}\mathbf{0 . 0 0 E} \\
+\mathbf{0 0}\end{array}$ \\
\hline & Std & $\begin{array}{r}9.22 E \\
-02\end{array}$ & $\begin{array}{r}1.76 E \\
-01\end{array}$ & $\begin{array}{r}1.75 E \\
-06\end{array}$ & $\begin{array}{r}1.03 E \\
-01\end{array}$ & $\begin{array}{r}3.64 E \\
-01\end{array}$ & $\begin{array}{r}\mathbf{0 . 0 0 E} \\
+00\end{array}$ & $\begin{array}{r}0.00 E \\
+00\end{array}$ & $\begin{array}{r}5.46 E \\
-02\end{array}$ & $\begin{array}{r}9.57 E \\
-02\end{array}$ & $\begin{array}{r}\mathbf{0 . 0 0 E} \\
+00\end{array}$ \\
\hline \multirow[t]{2}{*}{ F22 } & Mean & $\begin{array}{r}\mathbf{0 . 0 0 E} \\
+\mathbf{0 0}\end{array}$ & $\begin{array}{r}1.86 E \\
-02\end{array}$ & $\begin{array}{r}4.70 E \\
-03\end{array}$ & $\begin{array}{r}1.58 E \\
-02\end{array}$ & $\begin{array}{r}9.47 E \\
-02\end{array}$ & $\begin{array}{r}4.93 E \\
-01\end{array}$ & $\begin{array}{r}1.80 E \\
-01\end{array}$ & $\begin{array}{r}3.88 E \\
-48\end{array}$ & $\begin{array}{r}1.28 E \\
-03\end{array}$ & $\begin{array}{r}1.10 E \\
-01\end{array}$ \\
\hline & Std & $\begin{array}{r}\mathbf{0 . 0 0 E} \\
+\mathbf{0 0}\end{array}$ & $\begin{array}{r}3.04 E \\
-02\end{array}$ & $\begin{array}{r}1.85 E \\
-02\end{array}$ & $\begin{array}{r}2.62 E \\
-02\end{array}$ & $\begin{array}{r}8.69 E \\
-02\end{array}$ & $\begin{array}{r}5.21 E \\
-02\end{array}$ & $\begin{array}{r}4.07 E \\
-02\end{array}$ & $\begin{array}{r}1.75 E \\
-47\end{array}$ & $\begin{array}{r}1.75 E \\
-03\end{array}$ & $\begin{array}{r}7.12 E \\
-02\end{array}$ \\
\hline \multirow[t]{2}{*}{ F23 } & Mean & $\begin{array}{r}\mathbf{0 . 0 0 E} \\
+\mathbf{0 0}\end{array}$ & $\begin{array}{r}9.02 E \\
-03\end{array}$ & $\begin{array}{r}4.04 E \\
-05\end{array}$ & $\begin{array}{r}5.49 E \\
-03\end{array}$ & $\begin{array}{r}5.01 E \\
-02\end{array}$ & $\begin{array}{r}4.70 E \\
-01\end{array}$ & $\begin{array}{r}1.04 E \\
-01\end{array}$ & $\begin{array}{r}0.00 E \\
+00\end{array}$ & $\begin{array}{r}1.58 E \\
-05\end{array}$ & $\begin{array}{r}1.08 E \\
-01\end{array}$ \\
\hline & Std & $\begin{array}{r}\mathbf{0 . 0 0 E} \\
+\mathbf{0 0}\end{array}$ & $\begin{array}{r}1.56 E \\
-02\end{array}$ & $\begin{array}{r}1.25 E \\
-04\end{array}$ & $\begin{array}{r}9.54 E \\
-03\end{array}$ & $\begin{array}{r}5.35 E \\
-02\end{array}$ & $\begin{array}{r}1.70 E \\
-02\end{array}$ & $\begin{array}{r}1.11 E \\
-01\end{array}$ & $\begin{array}{r}0.00 E \\
+00\end{array}$ & $\begin{array}{r}3.26 E \\
-05\end{array}$ & $\begin{array}{r}1.19 E \\
-01\end{array}$ \\
\hline
\end{tabular}

diversity changes under the influence of different algorithmic mechanisms As the iterations run, the search agents traverse the whole search space and the average distance gradually becomes smaller, making the diversity decrease, and the algorithms gradually turn to the exploitation, which is consistent with the above analysis. In general, the diversity of MGEHO is significantly enhanced based on EHO. The reason lies in the additional exploration and variation strategies applied in MGEHO. These strategies lead to an enhanced level of diversity in the population, and therefore the probability of the algorithm falling into a locally optimal solution during the iterations is reduced.

\subsubsection{Sensitivity analysis on high-dimensional problems}

To further evaluate the effectiveness of MGEHO when optimizing complex high-dimensional problems, 23 benchmarks of F1-F23 are expanded to two higher dimension types, 500, 1000. The varying dimensions are set to evaluate the dimension impact on the algorithm. Under the consideration of fairness, other conditions 
Table 10 Comparison of results obtained on the F1-F23 benchmark functions $(d=200)$

\begin{tabular}{|c|c|c|c|c|c|c|c|c|c|c|c|}
\hline Function & & MGEHO & $\mathrm{EHO}$ & EEHO15 & OEHO & LFEHO & IGWO & EO & $\mathrm{HHO}$ & SFO & WOA \\
\hline \multirow[t]{2}{*}{ F1 } & Mean & $\begin{array}{r}0.00 E \\
+00\end{array}$ & $\begin{array}{r}8.88 E \\
-04\end{array}$ & $\begin{array}{r}2.67 E \\
-04\end{array}$ & $\begin{array}{r}1.91 E \\
-03\end{array}$ & $\begin{array}{r}1.09 E \\
-01\end{array}$ & $\begin{array}{r}1.29 E \\
-02\end{array}$ & $\begin{array}{r}1.64 E \\
-22\end{array}$ & $\begin{array}{r}1.92 E \\
-95\end{array}$ & $\begin{array}{r}8.32 E \\
-10\end{array}$ & $\begin{array}{r}8.88 E \\
-70\end{array}$ \\
\hline & Std & $\begin{array}{r}0.00 E \\
+00\end{array}$ & $\begin{array}{r}1.43 E \\
-03\end{array}$ & $\begin{array}{r}7.05 E \\
-04\end{array}$ & $\begin{array}{r}2.56 E \\
-03\end{array}$ & $\begin{array}{r}2.35 E \\
-01\end{array}$ & $\begin{array}{r}5.16 E \\
-03\end{array}$ & $\begin{array}{r}3.32 E \\
-22\end{array}$ & $\begin{array}{r}8.02 E \\
-95\end{array}$ & $\begin{array}{r}1.33 E \\
-09\end{array}$ & $\begin{array}{r}3.42 E \\
-69\end{array}$ \\
\hline \multirow[t]{2}{*}{$\mathrm{F} 2$} & Mean & $\begin{array}{r}0.00 E \\
+00\end{array}$ & $\begin{array}{r}4.67 E \\
-04\end{array}$ & $\begin{array}{r}6.73 E \\
-05\end{array}$ & $\begin{array}{r}8.18 E \\
-03\end{array}$ & $\begin{array}{r}5.01 E \\
-02\end{array}$ & $\begin{array}{r}2.48 E \\
-07\end{array}$ & $\begin{array}{r}8.41 E \\
-26\end{array}$ & $\begin{array}{r}3.78 E \\
-92\end{array}$ & $\begin{array}{r}2.84 E \\
-08\end{array}$ & $\begin{array}{r}5.14 E \\
-71\end{array}$ \\
\hline & Std & $\begin{array}{r}0.00 E \\
+00\end{array}$ & $\begin{array}{r}9.95 E \\
-04\end{array}$ & $\begin{array}{r}2.28 E \\
-04\end{array}$ & $\begin{array}{r}3.28 E \\
-02\end{array}$ & $\begin{array}{r}6.65 E \\
-02\end{array}$ & $\begin{array}{r}1.61 E \\
-07\end{array}$ & $\begin{array}{r}1.60 E \\
-25\end{array}$ & $\begin{array}{r}2.07 E \\
-91\end{array}$ & $\begin{array}{r}3.61 E \\
-08\end{array}$ & $\begin{array}{r}2.51 E \\
-70\end{array}$ \\
\hline \multirow[t]{2}{*}{ F3 } & Mean & $\begin{array}{c}8.14 E \\
-296\end{array}$ & $\begin{array}{r}3.29 E \\
-02\end{array}$ & $\begin{array}{r}5.96 E \\
-03\end{array}$ & $\begin{array}{r}5.95 E \\
-02\end{array}$ & $\begin{array}{r}1.98 E \\
-01\end{array}$ & $\begin{array}{r}1.67 E \\
-05\end{array}$ & $\begin{array}{r}1.71 E \\
-15\end{array}$ & $\begin{array}{r}3.67 E \\
-51\end{array}$ & $\begin{array}{r}3.12 E \\
-04\end{array}$ & $\begin{array}{r}1.02 E \\
-47\end{array}$ \\
\hline & Std & $\begin{array}{r}\mathbf{0 . 0 0 E} \\
+00\end{array}$ & $\begin{array}{r}4.06 E \\
-02\end{array}$ & $\begin{array}{r}9.22 E \\
-03\end{array}$ & $\begin{array}{r}5.82 E \\
-02\end{array}$ & $\begin{array}{r}1.92 E \\
-01\end{array}$ & $\begin{array}{r}4.71 E \\
-06\end{array}$ & $\begin{array}{r}1.28 E \\
-15\end{array}$ & $\begin{array}{r}9.51 E \\
-51\end{array}$ & $\begin{array}{r}3.17 E \\
-04\end{array}$ & $\begin{array}{r}5.54 E \\
-47\end{array}$ \\
\hline \multirow[t]{2}{*}{ F4 } & Mean & $\begin{array}{r}0.00 E \\
+00\end{array}$ & $\begin{array}{r}5.88 E \\
+00\end{array}$ & $\begin{array}{r}1.12 E \\
+00\end{array}$ & $\begin{array}{r}1.93 E \\
+01\end{array}$ & $\begin{array}{r}9.24 E \\
+01\end{array}$ & $\begin{array}{r}7.60 E \\
+04\end{array}$ & $\begin{array}{r}9.27 E \\
+02\end{array}$ & $\begin{array}{r}1.76 E \\
-45\end{array}$ & $\begin{array}{r}1.05 E \\
-05\end{array}$ & $\begin{array}{r}4.57 E \\
+06\end{array}$ \\
\hline & Std & $\begin{array}{r}0.00 E \\
+00\end{array}$ & $\begin{array}{r}1.22 E \\
+01\end{array}$ & $\begin{array}{r}3.45 E \\
+00\end{array}$ & $\begin{array}{r}6.54 E \\
+01\end{array}$ & $\begin{array}{r}1.48 E \\
+02\end{array}$ & $\begin{array}{r}1.56 E \\
+04\end{array}$ & $\begin{array}{r}1.22 E \\
+03\end{array}$ & $\begin{array}{r}9.24 E \\
-45\end{array}$ & $\begin{array}{r}2.61 E \\
-05\end{array}$ & $\begin{array}{r}1.18 E \\
+06\end{array}$ \\
\hline \multirow[t]{2}{*}{ F5 } & Mean & $\begin{array}{c}4.87 E \\
-297\end{array}$ & $\begin{array}{r}1.60 E \\
-03\end{array}$ & $\begin{array}{r}2.35 E \\
-04\end{array}$ & $\begin{array}{r}1.01 E \\
-03\end{array}$ & $\begin{array}{r}1.31 E \\
-02\end{array}$ & $\begin{array}{r}3.12 E \\
+01\end{array}$ & $\begin{array}{r}2.94 E \\
+01\end{array}$ & $\begin{array}{r}3.96 E \\
-48\end{array}$ & $\begin{array}{r}1.18 E \\
-06\end{array}$ & $\begin{array}{r}8.16 E \\
+01\end{array}$ \\
\hline & Std & $\begin{array}{r}0.00 E \\
+00\end{array}$ & $\begin{array}{r}1.48 E \\
-03\end{array}$ & $\begin{array}{r}2.21 E \\
-04\end{array}$ & $\begin{array}{r}1.12 E \\
-03\end{array}$ & $\begin{array}{r}1.43 E \\
-02\end{array}$ & $\begin{array}{r}5.43 E \\
+00\end{array}$ & $\begin{array}{r}2.74 E \\
+01\end{array}$ & $\begin{array}{r}1.37 E \\
-47\end{array}$ & $\begin{array}{r}1.07 E \\
-06\end{array}$ & $\begin{array}{r}1.98 E \\
+01\end{array}$ \\
\hline \multirow[t]{2}{*}{ F6 } & Mean & $\begin{array}{r}5.16 E \\
+00\end{array}$ & $\begin{array}{r}8.81 E \\
-01\end{array}$ & $\begin{array}{r}3.22 E \\
-01\end{array}$ & $\begin{array}{r}9.04 E \\
-01\end{array}$ & $\begin{array}{r}5.08 E \\
+01\end{array}$ & $\begin{array}{r}1.98 E \\
+02\end{array}$ & $\begin{array}{r}1.97 E \\
+02\end{array}$ & $\begin{array}{r}8.22 \mathrm{E} \\
-02\end{array}$ & $\begin{array}{r}1.47 E \\
-01\end{array}$ & $\begin{array}{r}1.98 E \\
+02\end{array}$ \\
\hline & Std & $\begin{array}{r}1.06 E \\
+01\end{array}$ & $\begin{array}{r}2.62 E \\
+00\end{array}$ & $\begin{array}{r}3.02 E \\
-01\end{array}$ & $\begin{array}{r}1.58 E \\
+00\end{array}$ & $\begin{array}{r}7.17 E \\
+01\end{array}$ & $\begin{array}{r}5.11 E \\
-01\end{array}$ & $\begin{array}{r}8.04 E \\
-01\end{array}$ & $\begin{array}{r}1.09 E \\
-01\end{array}$ & $\begin{array}{r}1.32 E \\
-01\end{array}$ & $\begin{array}{r}1.73 E \\
-01\end{array}$ \\
\hline \multirow[t]{2}{*}{ F7 } & Mean & $\begin{array}{r}1.21 E \\
-01\end{array}$ & $\begin{array}{r}9.45 E \\
-03\end{array}$ & $\begin{array}{r}1.94 E \\
-03\end{array}$ & $\begin{array}{r}7.17 E \\
-03\end{array}$ & $\begin{array}{r}2.91 E \\
+00\end{array}$ & $\begin{array}{r}2.64 E \\
+01\end{array}$ & $\begin{array}{r}2.04 E \\
+01\end{array}$ & $\begin{array}{r}8.92 E \\
-04\end{array}$ & $\begin{array}{r}5.34 E \\
-02\end{array}$ & $\begin{array}{r}1.09 E \\
+01\end{array}$ \\
\hline & Std & $\begin{array}{r}3.02 E \\
-01\end{array}$ & $\begin{array}{r}1.53 E \\
-02\end{array}$ & $\begin{array}{r}2.96 E \\
-03\end{array}$ & $\begin{array}{r}1.66 E \\
-02\end{array}$ & $\begin{array}{r}4.27 E \\
+00\end{array}$ & $\begin{array}{r}1.46 E \\
+00\end{array}$ & $\begin{array}{r}1.25 E \\
+00\end{array}$ & $\begin{array}{r}1.27 E \\
-03\end{array}$ & $\begin{array}{r}1.79 E \\
-01\end{array}$ & $\begin{array}{r}2.87 E \\
+00\end{array}$ \\
\hline \multirow[t]{2}{*}{ F8 } & Mean & $\begin{array}{r}0.00 E \\
+00\end{array}$ & $\begin{array}{r}6.79 E \\
-14\end{array}$ & $\begin{array}{r}4.67 E \\
-16\end{array}$ & $\begin{array}{r}1.65 E \\
-09\end{array}$ & $\begin{array}{r}1.02 E \\
-09\end{array}$ & $\begin{array}{r}9.44 E \\
-13\end{array}$ & $\begin{array}{r}7.06 E \\
-43\end{array}$ & $\begin{array}{r}3.18 E \\
-189\end{array}$ & $\begin{array}{r}6.07 E \\
-18\end{array}$ & $\begin{array}{l}1.54 E \\
-105\end{array}$ \\
\hline & Std & $\begin{array}{r}0.00 E \\
+00\end{array}$ & $\begin{array}{r}2.92 E \\
-13\end{array}$ & $\begin{array}{r}1.63 E \\
-15\end{array}$ & $\begin{array}{r}3.68 E \\
-09\end{array}$ & $\begin{array}{r}3.94 E \\
-09\end{array}$ & $\begin{array}{r}2.00 E \\
-12\end{array}$ & $\begin{array}{r}3.51 E \\
-42\end{array}$ & $\begin{array}{r}\mathbf{0 . 0 0 E} \\
+00\end{array}$ & $\begin{array}{r}3.31 E \\
-17\end{array}$ & $\begin{array}{l}8.44 E \\
-105\end{array}$ \\
\hline \multirow[t]{2}{*}{ F9 } & Mean & $\begin{array}{r}7.52 E \\
-05\end{array}$ & $\begin{array}{r}3.42 E \\
-03\end{array}$ & $\begin{array}{r}2.80 E \\
-03\end{array}$ & $\begin{array}{r}2.84 E \\
-03\end{array}$ & $\begin{array}{r}7.21 E \\
-03\end{array}$ & $\begin{array}{r}3.34 E \\
-02\end{array}$ & $\begin{array}{r}3.24 E \\
-03\end{array}$ & $\begin{array}{r}1.39 E \\
-04\end{array}$ & $\begin{array}{r}6.24 E \\
-04\end{array}$ & $\begin{array}{r}3.77 E \\
-03\end{array}$ \\
\hline & Std & $\begin{array}{r}8.54 \mathrm{E} \\
-05\end{array}$ & $\begin{array}{r}5.10 E \\
-03\end{array}$ & $\begin{array}{r}2.74 E \\
-03\end{array}$ & $\begin{array}{r}6.27 E \\
-03\end{array}$ & $\begin{array}{r}9.01 E \\
-03\end{array}$ & $\begin{array}{r}8.59 E \\
-03\end{array}$ & $\begin{array}{r}1.07 E \\
-03\end{array}$ & $\begin{array}{r}8.56 E \\
-05\end{array}$ & $\begin{array}{r}5.88 E \\
-04\end{array}$ & $\begin{array}{r}3.10 E \\
-03\end{array}$ \\
\hline \multirow[t]{2}{*}{ F10 } & Mean & $\begin{array}{r}0.00 E \\
+00\end{array}$ & $\begin{array}{r}6.41 E \\
-10\end{array}$ & $\begin{array}{r}3.72 E \\
-11\end{array}$ & $\begin{array}{r}9.22 E \\
-08\end{array}$ & $\begin{array}{r}2.29 E \\
-09\end{array}$ & $\begin{array}{r}1.28 E \\
-35\end{array}$ & $\begin{array}{c}1.15 E \\
-104\end{array}$ & $\begin{array}{l}1.09 E \\
-122\end{array}$ & $\begin{array}{r}5.10 E \\
-13\end{array}$ & $\begin{array}{l}1.53 E \\
-107\end{array}$ \\
\hline & Std & $\begin{array}{r}\text { 0.00E } \\
+00\end{array}$ & $\begin{array}{r}1.35 E \\
-09\end{array}$ & $\begin{array}{r}1.08 E \\
-10\end{array}$ & $\begin{array}{r}1.44 E \\
-07\end{array}$ & $\begin{array}{r}4.63 E \\
-09\end{array}$ & $\begin{array}{r}7.03 E \\
-35\end{array}$ & $\begin{array}{l}6.32 E \\
-104\end{array}$ & $\begin{array}{r}4.56 E \\
-122\end{array}$ & $\begin{array}{r}1.47 E \\
-12\end{array}$ & $\begin{array}{l}6.19 E \\
-107\end{array}$ \\
\hline \multirow[t]{2}{*}{ F11 } & Mean & $\begin{array}{c}-8.37 E \\
+04\end{array}$ & $\begin{array}{c}-8.38 E \\
+04\end{array}$ & $\begin{array}{c}-8.38 \mathrm{E} \\
+04\end{array}$ & $\begin{array}{c}-8.38 E \\
+04\end{array}$ & $\begin{array}{c}-8.38 E \\
+04\end{array}$ & $\begin{array}{c}-2.82 E \\
+04\end{array}$ & $\begin{array}{c}-4.29 E \\
+04\end{array}$ & $\begin{array}{c}-8.37 E \\
+04\end{array}$ & $\begin{array}{c}-9.91 E \\
+03\end{array}$ & $\begin{array}{l}-6.90 E \\
+04\end{array}$ \\
\hline & Std & $\begin{array}{r}1.22 E \\
+02\end{array}$ & $\begin{array}{r}8.68 E \\
+01\end{array}$ & $\begin{array}{r}3.31 E \\
+00\end{array}$ & $\begin{array}{r}3.61 E \\
+01\end{array}$ & $\begin{array}{r}5.21 E \\
+01\end{array}$ & $\begin{array}{r}1.40 E \\
+04\end{array}$ & $\begin{array}{r}2.69 E \\
+03\end{array}$ & $\begin{array}{r}3.43 E \\
+02\end{array}$ & $\begin{array}{r}7.11 E \\
+02\end{array}$ & $\begin{array}{r}1.34 E \\
+04\end{array}$ \\
\hline \multirow[t]{2}{*}{$\mathrm{F} 12$} & Mean & $\begin{array}{r}0.00 \mathrm{E} \\
+\mathbf{0 0}\end{array}$ & $\begin{array}{r}4.55 E \\
-04\end{array}$ & $\begin{array}{r}9.20 E \\
-06\end{array}$ & $\begin{array}{r}5.98 E \\
-03\end{array}$ & $\begin{array}{r}1.01 E \\
-01\end{array}$ & $\begin{array}{r}3.05 E \\
+02\end{array}$ & $\begin{array}{r}0.00 E \\
+00\end{array}$ & $\begin{array}{r}0.00 E \\
+00\end{array}$ & $\begin{array}{r}3.41 E \\
-06\end{array}$ & $\begin{array}{r}1.52 E \\
-14\end{array}$ \\
\hline & Std & $\begin{array}{r}0.00 E \\
+00\end{array}$ & $\begin{array}{r}8.89 E \\
-04\end{array}$ & $\begin{array}{r}2.10 E \\
-05\end{array}$ & $\begin{array}{r}9.91 E \\
-03\end{array}$ & $\begin{array}{r}2.91 E \\
-01\end{array}$ & $\begin{array}{r}8.73 E \\
+01\end{array}$ & $\begin{array}{r}0.00 E \\
+00\end{array}$ & $\begin{array}{r}0.00 E \\
+00\end{array}$ & $\begin{array}{r}8.23 E \\
-06\end{array}$ & $\begin{array}{r}8.30 E \\
-14\end{array}$ \\
\hline \multirow[t]{2}{*}{$\mathrm{F} 13$} & Mean & $\begin{array}{r}8.88 E \\
-16\end{array}$ & $\begin{array}{r}2.32 E \\
-03\end{array}$ & $\begin{array}{r}2.28 E \\
-04\end{array}$ & $\begin{array}{r}1.46 E \\
-03\end{array}$ & $\begin{array}{r}1.51 E \\
-02\end{array}$ & $\begin{array}{r}4.29 E \\
-05\end{array}$ & $\begin{array}{r}1.05 E \\
-13\end{array}$ & $\begin{array}{r}8.88 E \\
-16\end{array}$ & $\begin{array}{r}3.21 E \\
-06\end{array}$ & $\begin{array}{r}3.49 E \\
-15\end{array}$ \\
\hline & Std & $\begin{array}{r}0.00 E \\
+00\end{array}$ & $\begin{array}{r}2.59 E \\
-03\end{array}$ & $\begin{array}{r}3.77 E \\
-04\end{array}$ & $\begin{array}{r}1.20 E \\
-03\end{array}$ & $\begin{array}{r}1.46 E \\
-02\end{array}$ & $\begin{array}{r}1.19 E \\
-05\end{array}$ & $\begin{array}{r}2.93 E \\
-14\end{array}$ & $\begin{array}{r}0.00 E \\
+00\end{array}$ & $\begin{array}{r}3.57 E \\
-06\end{array}$ & $\begin{array}{r}2.63 E \\
-15\end{array}$ \\
\hline
\end{tabular}


Table 10 (continued)

\begin{tabular}{|c|c|c|c|c|c|c|c|c|c|c|c|}
\hline Function & & MGEHO & EHO & EEHO15 & OEHO & LFEHO & IGWO & EO & $\mathrm{HHO}$ & SFO & WOA \\
\hline \multirow[t]{2}{*}{ F14 } & Mean & $\begin{array}{r}\mathbf{0 . 0 0 E} \\
+\mathbf{0 0}\end{array}$ & $\begin{array}{r}4.54 E \\
-04\end{array}$ & $\begin{array}{r}3.64 E \\
-05\end{array}$ & $\begin{array}{r}9.25 E \\
-05\end{array}$ & $\begin{array}{r}1.84 E \\
-02\end{array}$ & $\begin{array}{r}4.39 E \\
-03\end{array}$ & $\begin{array}{r}0.00 E \\
+00\end{array}$ & $\begin{array}{r}0.00 E \\
+00\end{array}$ & $\begin{array}{r}1.75 E \\
-11\end{array}$ & $\begin{array}{r}0.00 E \\
+00\end{array}$ \\
\hline & Std & $\begin{array}{r}0.00 E \\
+00\end{array}$ & $\begin{array}{r}1.03 E \\
-03\end{array}$ & $\begin{array}{r}1.26 E \\
-04\end{array}$ & $\begin{array}{r}1.30 E \\
-04\end{array}$ & $\begin{array}{r}2.41 E \\
-02\end{array}$ & $\begin{array}{r}9.35 E \\
-03\end{array}$ & $\begin{array}{r}0.00 E \\
+00\end{array}$ & $\begin{array}{r}\text { 0.00E } \\
+00\end{array}$ & $\begin{array}{r}4.33 E \\
-11\end{array}$ & $\begin{array}{r}\text { 0.00E } \\
+00\end{array}$ \\
\hline \multirow[t]{2}{*}{ F15 } & Mean & $\begin{array}{r}5.43 E \\
-05\end{array}$ & $\begin{array}{r}9.31 E \\
-06\end{array}$ & $\begin{array}{r}8.41 E \\
-06\end{array}$ & $\begin{array}{r}1.21 E \\
-05\end{array}$ & $\begin{array}{r}1.77 E \\
-03\end{array}$ & $\begin{array}{r}6.07 E \\
+00\end{array}$ & $\begin{array}{r}2.14 E \\
-01\end{array}$ & $\begin{array}{r}4.06 \mathrm{E} \\
-06\end{array}$ & $\begin{array}{r}1.39 E \\
-02\end{array}$ & $\begin{array}{r}7.16 E \\
-02\end{array}$ \\
\hline & Std & $\begin{array}{r}1.31 E \\
-04\end{array}$ & $\begin{array}{r}1.46 E \\
-05\end{array}$ & $\begin{array}{r}8.37 E \\
-06\end{array}$ & $\begin{array}{r}2.42 E \\
-05\end{array}$ & $\begin{array}{r}3.57 E \\
-03\end{array}$ & $\begin{array}{r}5.69 E \\
+00\end{array}$ & $\begin{array}{r}3.53 E \\
-02\end{array}$ & $\begin{array}{r}6.33 E \\
-06\end{array}$ & $\begin{array}{r}4.54 E \\
-02\end{array}$ & $\begin{array}{r}2.98 E \\
-02\end{array}$ \\
\hline \multirow[t]{2}{*}{ F16 } & Mean & $\begin{array}{r}1.98 E \\
-03\end{array}$ & $\begin{array}{r}2.71 E \\
-03\end{array}$ & $\begin{array}{r}7.21 E \\
-04\end{array}$ & $\begin{array}{r}1.20 E \\
-03\end{array}$ & $\begin{array}{r}2.60 E \\
-01\end{array}$ & $\begin{array}{r}2.13 E \\
+01\end{array}$ & $\begin{array}{r}1.84 E \\
+01\end{array}$ & $\begin{array}{r}2.13 E \\
-04\end{array}$ & $\begin{array}{r}2.73 E \\
-04\end{array}$ & $\begin{array}{r}7.04 E \\
+00\end{array}$ \\
\hline & Std & $\begin{array}{r}3.40 E \\
-03\end{array}$ & $\begin{array}{r}7.21 E \\
-03\end{array}$ & $\begin{array}{r}9.95 E \\
-04\end{array}$ & $\begin{array}{r}4.59 E \\
-03\end{array}$ & $\begin{array}{r}5.63 E \\
-01\end{array}$ & $\begin{array}{r}2.90 E \\
+00\end{array}$ & $\begin{array}{r}4.33 E \\
-01\end{array}$ & $\begin{array}{r}2.11 E \\
-04\end{array}$ & $\begin{array}{r}2.28 E \\
-04\end{array}$ & $\begin{array}{r}2.20 E \\
+00\end{array}$ \\
\hline \multirow[t]{2}{*}{ F17 } & Mean & $\begin{array}{c}1.08 E \\
-278\end{array}$ & $\begin{array}{r}2.03 E \\
-03\end{array}$ & $\begin{array}{r}3.80 E \\
-04\end{array}$ & $\begin{array}{r}3.33 E \\
-03\end{array}$ & $\begin{array}{r}1.37 E \\
-02\end{array}$ & $\begin{array}{r}1.87 E \\
-02\end{array}$ & $\begin{array}{r}2.33 E \\
-16\end{array}$ & $\begin{array}{r}6.12 E \\
-05\end{array}$ & $\begin{array}{r}3.15 E \\
-05\end{array}$ & $\begin{array}{r}3.87 E \\
-50\end{array}$ \\
\hline & Std & $\begin{array}{r}0.00 E \\
+00\end{array}$ & $\begin{array}{r}2.40 E \\
-03\end{array}$ & $\begin{array}{r}6.76 E \\
-04\end{array}$ & $\begin{array}{r}3.97 E \\
-03\end{array}$ & $\begin{array}{r}1.45 E \\
-02\end{array}$ & $\begin{array}{r}8.60 E \\
-03\end{array}$ & $\begin{array}{r}1.58 E \\
-16\end{array}$ & $\begin{array}{r}2.22 E \\
-04\end{array}$ & $\begin{array}{r}5.14 E \\
-05\end{array}$ & $\begin{array}{r}1.48 E \\
-49\end{array}$ \\
\hline \multirow[t]{2}{*}{ F18 } & Mean & $\begin{array}{c}-1.42 \mathrm{E} \\
-14\end{array}$ & $\begin{array}{r}2.04 E \\
-06\end{array}$ & $\begin{array}{r}7.21 E \\
-08\end{array}$ & $\begin{array}{r}6.50 E \\
-04\end{array}$ & $\begin{array}{r}2.30 E \\
-04\end{array}$ & $\begin{array}{r}2.33 E \\
-10\end{array}$ & $\begin{array}{c}-1.42 E \\
-14\end{array}$ & $\begin{array}{c}-1.42 E \\
-14\end{array}$ & $\begin{array}{r}1.53 E \\
-08\end{array}$ & $\begin{array}{c}-1.42 E \\
-14\end{array}$ \\
\hline & Std & $\begin{array}{r}0.00 E \\
+00\end{array}$ & $\begin{array}{r}6.15 E \\
-06\end{array}$ & $\begin{array}{r}1.17 E \\
-07\end{array}$ & $\begin{array}{r}1.40 E \\
-03\end{array}$ & $\begin{array}{r}4.01 E \\
-04\end{array}$ & $\begin{array}{r}1.40 E \\
-10\end{array}$ & $\begin{array}{r}\mathbf{0 . 0 0 E} \\
+\mathbf{0 0}\end{array}$ & $\begin{array}{r}\text { 0.00E } \\
+\mathbf{0 0}\end{array}$ & $\begin{array}{r}1.73 E \\
-08\end{array}$ & $\begin{array}{r}\mathbf{0 . 0 0 E} \\
+\mathbf{0 0}\end{array}$ \\
\hline \multirow[t]{2}{*}{ F19 } & Mean & $\begin{array}{r}\mathbf{0 . 0 0 E} \\
+\mathbf{0 0}\end{array}$ & $\begin{array}{r}4.90 E \\
+00\end{array}$ & $\begin{array}{r}2.03 E \\
+00\end{array}$ & $\begin{array}{r}6.45 E \\
+00\end{array}$ & $\begin{array}{r}1.18 E \\
+01\end{array}$ & $\begin{array}{r}3.42 \mathrm{E} \\
+00\end{array}$ & $\begin{array}{r}4.61 E \\
-07\end{array}$ & $\begin{array}{r}1.46 E \\
-24\end{array}$ & $\begin{array}{r}5.46 E \\
-01\end{array}$ & $\begin{array}{r}4.25 E \\
-29\end{array}$ \\
\hline & Std & $\begin{array}{r}\mathbf{0 . 0 0 E} \\
+00\end{array}$ & $\begin{array}{r}3.36 E \\
+00\end{array}$ & $\begin{array}{r}1.09 E \\
+00\end{array}$ & $\begin{array}{r}4.48 E \\
+00\end{array}$ & $\begin{array}{r}6.92 E \\
+00\end{array}$ & $\begin{array}{r}7.36 \mathrm{E} \\
-01\end{array}$ & $\begin{array}{r}1.58 E \\
-07\end{array}$ & $\begin{array}{r}6.10 E \\
-24\end{array}$ & $\begin{array}{r}3.78 E \\
-01\end{array}$ & $\begin{array}{r}1.43 E \\
-28\end{array}$ \\
\hline \multirow[t]{2}{*}{ F20 } & Mean & $\begin{array}{r}0.00 E \\
+00\end{array}$ & $\begin{array}{r}1.08 E \\
+01\end{array}$ & $\begin{array}{r}7.51 E \\
-01\end{array}$ & $\begin{array}{r}3.24 E \\
+01\end{array}$ & $\begin{array}{r}3.46 E \\
+03\end{array}$ & $\begin{array}{r}3.79 E \\
-04\end{array}$ & $\begin{array}{r}5.28 E \\
-22\end{array}$ & $\begin{array}{r}1.99 E \\
-92\end{array}$ & $\begin{array}{r}2.01 E \\
-05\end{array}$ & $\begin{array}{r}1.18 E \\
-67\end{array}$ \\
\hline & Std & $\begin{array}{r}0.00 E \\
+00\end{array}$ & $\begin{array}{r}1.87 E \\
+01\end{array}$ & $\begin{array}{r}1.67 E \\
+00\end{array}$ & $\begin{array}{r}1.06 E \\
+02\end{array}$ & $\begin{array}{r}5.82 E \\
+03\end{array}$ & $\begin{array}{r}2.11 E \\
-04\end{array}$ & $\begin{array}{r}6.99 E \\
-22\end{array}$ & $\begin{array}{r}9.48 E \\
-92\end{array}$ & $\begin{array}{r}2.45 E \\
-05\end{array}$ & $\begin{array}{r}4.23 E \\
-67\end{array}$ \\
\hline \multirow[t]{2}{*}{ F21 } & Mean & $\begin{array}{c}-7.72 E \\
-01\end{array}$ & $\begin{array}{c}-8.13 E \\
-01\end{array}$ & $\begin{array}{c}-1.00 E \\
+00\end{array}$ & $\begin{array}{c}-8.75 E \\
-01\end{array}$ & $\begin{array}{c}-4.94 E \\
-01\end{array}$ & $\begin{array}{r}\text { 0.00E } \\
+\mathbf{0 0}\end{array}$ & $\begin{array}{r}\text { 0.00E } \\
+\mathbf{0 0}\end{array}$ & $\begin{array}{c}-5.01 E \\
-01\end{array}$ & $\begin{array}{c}-8.48 E \\
-01\end{array}$ & $\begin{array}{r}\mathbf{0 . 0 0 E} \\
+00\end{array}$ \\
\hline & Std & $\begin{array}{r}3.10 E \\
-01\end{array}$ & $\begin{array}{r}2.79 E \\
-01\end{array}$ & $\begin{array}{r}1.04 E \\
-05\end{array}$ & $\begin{array}{r}2.28 E \\
-01\end{array}$ & $\begin{array}{r}3.61 E \\
-01\end{array}$ & $\begin{array}{r}0.00 E \\
+00\end{array}$ & $\begin{array}{r}0.00 E \\
+00\end{array}$ & $\begin{array}{r}4.70 E \\
-01\end{array}$ & $\begin{array}{r}1.81 E \\
-01\end{array}$ & $\begin{array}{r}\mathbf{0 . 0 0 E} \\
+00\end{array}$ \\
\hline \multirow[t]{2}{*}{ F22 } & Mean & $\begin{array}{r}\mathbf{0 . 0 0 E} \\
+\mathbf{0 0}\end{array}$ & $\begin{array}{r}2.70 E \\
-02\end{array}$ & $\begin{array}{r}2.28 E \\
-03\end{array}$ & $\begin{array}{r}2.04 E \\
-02\end{array}$ & $\begin{array}{r}9.41 E \\
-02\end{array}$ & $\begin{array}{r}7.77 E \\
-01\end{array}$ & $\begin{array}{r}2.00 E \\
-01\end{array}$ & $\begin{array}{r}3.49 E \\
-49\end{array}$ & $\begin{array}{r}1.35 E \\
-03\end{array}$ & $\begin{array}{r}1.17 E \\
-01\end{array}$ \\
\hline & Std & $\begin{array}{r}\mathbf{0 . 0 0 E} \\
+\mathbf{0 0}\end{array}$ & $\begin{array}{r}3.45 E \\
-02\end{array}$ & $\begin{array}{r}5.49 E \\
-03\end{array}$ & $\begin{array}{r}3.03 E \\
-02\end{array}$ & $\begin{array}{r}9.52 E \\
-02\end{array}$ & $\begin{array}{r}9.35 E \\
-02\end{array}$ & $\begin{array}{r}3.29 E \\
-10\end{array}$ & $\begin{array}{r}1.33 E \\
-48\end{array}$ & $\begin{array}{r}1.72 E \\
-03\end{array}$ & $\begin{array}{r}6.47 E \\
-02\end{array}$ \\
\hline \multirow[t]{2}{*}{ F23 } & Mean & $\begin{array}{r}\mathbf{0 . 0 0 E} \\
+\mathbf{0 0}\end{array}$ & $\begin{array}{r}1.08 E \\
-02\end{array}$ & $\begin{array}{r}1.06 E \\
-04\end{array}$ & $\begin{array}{r}7.32 E \\
-03\end{array}$ & $\begin{array}{r}7.92 E \\
-02\end{array}$ & $\begin{array}{r}4.97 E \\
-01\end{array}$ & $\begin{array}{r}2.41 E \\
-01\end{array}$ & $\begin{array}{r}\text { 0.00E } \\
+00\end{array}$ & $\begin{array}{r}6.43 E \\
-05\end{array}$ & $\begin{array}{r}8.93 E \\
-02\end{array}$ \\
\hline & Std & $\begin{array}{r}\mathbf{0 . 0 0 E} \\
+\mathbf{0 0}\end{array}$ & $\begin{array}{r}1.69 E \\
-02\end{array}$ & $\begin{array}{r}2.22 E \\
-04\end{array}$ & $\begin{array}{r}1.38 E \\
-02\end{array}$ & $\begin{array}{r}7.37 E \\
-02\end{array}$ & $\begin{array}{r}1.10 E \\
-03\end{array}$ & $\begin{array}{r}1.11 E \\
-01\end{array}$ & $\begin{array}{r}\mathbf{0 . 0 0 E} \\
+\mathbf{0 0}\end{array}$ & $\begin{array}{r}1.15 E \\
-04\end{array}$ & $\begin{array}{r}1.09 E \\
-01\end{array}$ \\
\hline
\end{tabular}

remain the same as in the previous experiments. Tables 14 and 15 shows the results of MGEHO with other algorithms of 23 benchmark functions on 500 and 1000 dimensions. The best solutions for each function are in bold. Moreover, based on the Friedman test in Table 16, the average ranking of MGEHO is 2.11 and 2.25 on 500 and 1000 dimensions, which are lower than the ranking results of all involved variants of the algorithm. From this aspect, it can be confirmed that MGEHO is superior than the above algorithms in optimizing complex high-dimensional problems.

\subsection{Experiments on CEC2014 and CEC2017 benchmark functions}

To further verify the performance of MGEHO when dealing with complex problems, experiments are conducted on the CEC2014 and CEC2017 composite benchmark functions. Besides, seven advanced metaheuristic algorithms were selected for comparison, they are the improved elephant herding optimization (EHOI) [21], the RUNge Kutta optimizer (RUN) [45], the gradient-based optimizer (GBO) [46], the Hunger Games Search (HGS) [47], the 
Table 11 Nonparametric statistical comparison based on the Wilcoxon signed-rank test for F1-F33

\begin{tabular}{|c|c|c|c|c|c|c|c|c|c|}
\hline Function & $\mathrm{HO}$ & EHO15 & OEHO & LFEHO & IGWO & EO & HHO & SFO & WOA \\
\hline 1 & $21 E-12$ & $1.21 E-12$ & $.21 E-12$ & $1.21 E-12$ & $1.21 E-12$ & $1.21 E-12$ & $1.21 E-12$ & $1.21 E-12$ & $1.21 E-1$ \\
\hline $\mathrm{F} 2$ & $.21 E-12$ & $1.21 E-12$ & $1.21 E-12$ & $1.21 E-12$ & $1.21 E-12$ & $1.21 E-12$ & $1.21 E-12$ & $1.21 E-12$ & $1.21 E-12$ \\
\hline 3 & $.02 E-11$ & $3.02 E-11$ & $3.02 E-11$ & $3.02 E-11$ & $3.02 E-11$ & $3.02 E-11$ & $3.02 E-11$ & $3.02 E-11$ & $3.02 E-11$ \\
\hline 4 & $21 E-12$ & $1.21 E-12$ & $1.21 E-12$ & $1.21 E-12$ & $1.21 E-12$ & $1.21 E-12$ & $1.21 E-12$ & $1.21 E-12$ & $1.21 E-12$ \\
\hline F5 & $02 E-11$ & $3.02 E-11$ & $3.02 E-11$ & $3.02 E-11$ & $3.02 E-11$ & $3.02 E-11$ & $3.02 E-11$ & $3.02 E-11$ & $3.02 E-11$ \\
\hline F6 & $84 E-04$ & $1.70 E-02$ & $5.30 E-06$ & $1.04 E-04$ & $5.57 E-10$ & $5.57 E-10$ & $5.86 E-06$ & $4.36 E-02$ & $3.02 E-11$ \\
\hline F7 & $06 \mathrm{E}-01$ & $9.88 E-03$ & $7.24 E-06$ & $1.07 E-07$ & $9.52 E-04$ & $1.07 E-09$ & $8.29 E-06$ & $6.97 E-03$ & $3.02 E-11$ \\
\hline F8 & $21 E-12$ & $1.21 E-12$ & $1.21 E-12$ & $1.21 E-12$ & $1.21 E-12$ & $1.21 E-12$ & $1.21 E-12$ & $1.21 E-12$ & $1.21 E-12$ \\
\hline F8 & $73 E-07$ & $8.15 E-11$ & $4.31 E-08$ & $4.18 E-09$ & $3.02 E-11$ & $3.02 E-11$ & $9.07 E-03$ & $2.03 E-07$ & $1.33 E-10$ \\
\hline F9 & $21 E-12$ & $1.21 E-12$ & $1.21 E-12$ & $1.21 E-12$ & $1.21 E-12$ & $1.21 E-12$ & $1.21 E-12$ & $1.21 E-12$ & $1.21 E-12$ \\
\hline F10 & $.79 E-03$ & $3.50 E-09$ & $7.48 E-06$ & $5.30 \mathrm{E}-01$ & $3.02 E-11$ & $3.02 E-11$ & $8.68 E-03$ & $3.02 E-11$ & $9.26 E-09$ \\
\hline F11 & $9.21 E-05$ & $1.26 E-06$ & $1.16 E-07$ & $2.68 E-06$ & $3.02 E-11$ & $3.02 E-11$ & $1.49 E-04$ & $3.02 E-11$ & $3.02 E-11$ \\
\hline F12 & $1.21 E-12$ & $1.21 E-12$ & $1.21 E-12$ & $1.21 E-12$ & $1.21 E-12$ & $\mathrm{NaN}$ & $\mathbf{N a N}$ & $1.21 E-12$ & $\mathrm{NaN}$ \\
\hline F13 & $.21 E-12$ & $1.21 E-12$ & $1.21 E-12$ & $1.21 E-12$ & $1.03 E-12$ & $8.71 E-14$ & $\mathrm{NaN}$ & $1.21 E-12$ & $1.02 E-07$ \\
\hline F14 & $.21 E-12$ & $1.21 E-12$ & $1.21 E-12$ & $1.21 E-12$ & $5.58 E-03$ & $\mathrm{NaN}$ & $\mathrm{NaN}$ & $1.21 E-12$ & $1.61 \mathrm{E}-01$ \\
\hline F15 & $2.71 E-02$ & $5.01 E-02$ & $7.73 E-02$ & $2.13 E-05$ & $7.01 E-02$ & $5.97 E-09$ & $1.27 E-02$ & $9.71 E-01$ & $1.33 E-10$ \\
\hline F16 & $3.79 E-04$ & $3.63 E-01$ & $6.20 \mathrm{E}-01$ & $2.00 E-06$ & $6.77 E-05$ & $9.82 E-01$ & $1.32 E-04$ & $3.01 E-04$ & $3.02 E-11$ \\
\hline F17 & $3.02 E-11$ & $3.02 E-11$ & $3.02 E-11$ & $3.02 E-11$ & $3.02 E-11$ & $3.02 E-11$ & $3.02 E-11$ & $3.02 E-11$ & $3.02 E-11$ \\
\hline F18 & $21 E-12$ & $1.21 E-12$ & $1.21 E-12$ & $1.21 E-12$ & $\mathrm{NaN}$ & $\mathrm{NaN}$ & $\mathrm{NaN}$ & $1.21 E-12$ & $3.34 E-01$ \\
\hline F19 & $21 E-12$ & $1.21 E-12$ & $1.21 E-12$ & $1.21 E-12$ & $1.21 E-12$ & $1.21 E-12$ & $1.21 E-12$ & $1.21 E-12$ & $1.21 E-12$ \\
\hline F20 & $21 E-12$ & $1.21 E-12$ & $1.21 E-12$ & $1.21 E-12$ & $1.21 E-12$ & $1.21 E-12$ & $1.21 E-12$ & $1.21 E-12$ & $1.21 E-12$ \\
\hline 21 & $64 E-05$ & $3.37 E-04$ & $6.41 E-05$ & $2.13 E-05$ & $1.21 E-12$ & $3.02 E-11$ & $2.51 E-02$ & $1.53 E-05$ & $1.21 E-12$ \\
\hline F22 & $21 E-12$ & $1.21 E-12$ & $1.21 E-12$ & $1.21 E-12$ & $1.21 E-12$ & $1.18 E-12$ & $1.21 E-12$ & $1.21 E-12$ & $1.21 E-12$ \\
\hline F23 & $1.21 E-12$ & $1.21 E-12$ & $1.21 E-12$ & $1.21 E-12$ & $1.21 E-12$ & $9.42 E-13$ & & $1.21 E-12$ & $3.87 E-12$ \\
\hline $\mathrm{F} 24$ & $4.46 \mathrm{E}-01$ & $1.17 E-09$ & $2.71 E-01$ & $1.33 E-02$ & $8.87 E-12$ & $3.11 E-10$ & $5.87 E-04$ & $3.02 E-11$ & $2.32 E-02$ \\
\hline $\mathrm{F} 25$ & $4.08 E-11$ & $1.55 E-09$ & $3.69 E-11$ & $8.20 E-07$ & $8.48 E-09$ & $4.92 E-01$ & $4.20 \mathrm{E}-01$ & $4.06 E-02$ & $4.12 E-06$ \\
\hline F26 & $3.02 E-11$ & $4.46 E-04$ & $3.02 E-11$ & $7.39 E-11$ & $1.01 E-11$ & $4.08 E-12$ & $3.02 E-11$ & $3.69 E-11$ & $3.02 E-11$ \\
\hline F27 & $5.00 E-09$ & $1.78 E-04$ & $1.10 E-08$ & $4.11 E-07$ & $1.72 E-12$ & $1.21 E-12$ & $4.98 E-11$ & 3.04E -01 & $7.39 E-11$ \\
\hline F28 & $3.02 E-11$ & $1.47 E-07$ & $3.02 E-11$ & $7.39 E-11$ & $2.41 E-11$ & $2.53 E-11$ & $3.02 E-11$ & $3.02 E-11$ & $3.01 E-0$ \\
\hline F29 & $3.02 E-11$ & $1.17 E-05$ & $3.02 E-11$ & $4.94 E-05$ & $1.41 E-11$ & $1.44 E-10$ & $4.46 E-04$ & $2.49 E-06$ & $3.95 E-0$ \\
\hline F30 & $3.02 E-11$ & $3.50 E-09$ & $3.02 E-11$ & $1.91 E-01$ & $7.04 E-07$ & $6.13 E-03$ & $1.11 E-04$ & $3.50 E-09$ & $1.09 \mathrm{E}-0$ \\
\hline F31 & $4.06 E-02$ & $1.87 E-05$ & $8.77 E-01$ & $1.37 E-01$ & $1.07 E-09$ & $7.70 E-03$ & $3.02 E-11$ & $5.09 E-08$ & $4.44 E-0$ \\
\hline F32 & $5.83 E-03$ & $1.58 E-04$ & $1.19 E-06$ & $2.81 E-02$ & $3.02 E-11$ & $8.69 E-07$ & $3.02 E-11$ & $1.07 E-09$ & $4.31 E-0$ \\
\hline F33 & $2.64 E-05$ & $1.61 E-06$ & $3.85 E-03$ & $6.67 E-03$ & $3.02 E-11$ & $3.81 E-04$ & $3.02 E-11$ & $8.10 E-10$ & $3.20 E-0$ \\
\hline$+l=I-$ & $31 / 2 / 0$ & $31 / 2 / 0$ & $29 / 4 / 0$ & $30 / 3 / 0$ & $31 / 2 / 0$ & $28 / 5 / 0$ & $27 / 6 / 0$ & $31 / 2 / 0$ & $29 / 4 / 0$ \\
\hline
\end{tabular}

Slime mould algorithm (SMA) [48], the an elite evolutionary strategy based Harris hawks optimization (EESHHO) [49], the multi-trial vector-based differential evolution (MTDE) [50]. It should be noted that the parameter settings of these algorithms are selected from the recent reported literature. Moreover, the population size $N$ for all algorithms is set to 30 , the spatial dimension is 30 , and the maximum number of iterations $t_{\max }$ is 1000 . The results are based on 30 runs.

\subsubsection{Benchmark functions}

In this subsection, 32 benchmark functions from CEC2014 and CEC2017 [51] are employed in this section to demonstrate the efficacy of MGEHO. The descriptions of these functions are presented in Table 17, where $D$ represents the dimension of the function and "search range" is the parameter that constrains the frontier of the search space of the corresponding function. In general, these 
Table 12 Nonparametric statistical comparison based on the Friedman rank test for F1-F23

\begin{tabular}{|c|c|c|c|c|c|c|c|c|c|c|c|c|c|c|c|}
\hline Function & Dimensionality & $\mathrm{F} 1$ & $\mathrm{~F} 2$ & $\mathrm{~F} 3$ & $\mathrm{~F} 4$ & F5 & & 86 & F7 & F8 & F9 & F10 & F11 & F12 & F13 \\
\hline \multirow[t]{3}{*}{ MGEHO } & 30 & 1 & 1 & 1 & 1 & 1 & & 4.5 & 6.4 & 1 & 1.63 & 1 & 4.57 & 1 & 1 \\
\hline & 100 & 1 & 1 & 1 & 1 & 1 & & 4.63 & 4.63 & 1 & 1.43 & 1 & 4.3 & 1 & 1 \\
\hline & 200 & 1 & 1 & 1 & 1 & 1 & & 4.6 & 5.07 & 1 & 1.7 & 1 & 4.4 & 1 & 1 \\
\hline \multirow[t]{3}{*}{ EHO } & 30 & 8.43 & 8.47 & 8.2 & 7.13 & 7.4 & & 4.43 & 5.43 & 7.7 & 5.33 & 8.37 & 3.97 & 7 & 8.6 \\
\hline & 100 & 8.57 & 8.37 & 8.1 & 5.87 & 5.77 & & 4.27 & 4.07 & 7.87 & 5.33 & 8.2 & 3.53 & 6.93 & 8.67 \\
\hline & 200 & 7.27 & 8.23 & 8.17 & 5.37 & 5.73 & & 3.97 & 4 & 6.83 & 5.37 & 8.1 & 3.97 & 6.93 & 8.5 \\
\hline \multirow[t]{3}{*}{ EEHO15 } & 30 & 7.5 & 7.27 & 7.17 & 6.03 & 6.4 & & 3.47 & 4.83 & 7 & 7.1 & 7.13 & 1.5 & 6 & 7.23 \\
\hline & 100 & 7.33 & 7.07 & 7.27 & 4.6 & 4.57 & & 3.27 & 3.53 & 6.83 & 6.73 & 6.93 & 1.7 & 5.93 & 7.3 \\
\hline & 200 & 6.47 & 7.37 & 7.27 & 4.4 & 4.27 & & 3.93 & 2.87 & 6 & 6.03 & 7.3 & 1.63 & 5.73 & 6.83 \\
\hline \multirow[t]{3}{*}{ OEHO } & 30 & 8.23 & 8.7 & 8.83 & 7.23 & 7.33 & & 4.1 & 5.37 & 9.5 & 5.47 & 9.87 & 3.77 & 8.03 & 8.47 \\
\hline & 100 & 8.33 & 8.97 & 8.9 & 6.1 & 5.9 & & 3.93 & 3.77 & 9.63 & 5.1 & 9.83 & 4.23 & 8.17 & 8.5 \\
\hline & 200 & 7.5 & 8.7 & 8.67 & 5.63 & 5.17 & & 4.13 & 3.6 & 9.17 & 4.57 & 9.77 & 3.67 & 8.07 & 8.5 \\
\hline \multirow[t]{3}{*}{ LFEHO } & 30 & 9.83 & 9.53 & 9.7 & 8.53 & 8.83 & & 5.5 & 8.77 & 9.33 & 6.23 & 8.43 & 4.17 & 8.6 & 9.67 \\
\hline & 100 & 9.77 & 9.5 & 9.63 & 7.53 & 7.87 & & 5.73 & 6.57 & 9.07 & 6.53 & 8.73 & 4.27 & 8.5 & 9.53 \\
\hline & 200 & 9.47 & 9.63 & 9.77 & 6.77 & 6.83 & & 5.87 & 6.73 & 9.3 & 7.07 & 8.67 & 4.1 & 8.73 & 9.83 \\
\hline \multirow[t]{3}{*}{ IGWO } & 30 & 5 & 5 & 5 & 6.03 & 5.03 & & 7.87 & 4.6 & 5 & 8.47 & 5 & 8.47 & 10 & 5 \\
\hline & 100 & 5.1 & 5 & 5 & 9 & 9 & & 9.17 & 10 & 5.37 & 9.73 & 5 & 8.87 & 10 & 5.03 \\
\hline & 200 & 9.3 & 6.07 & 5.1 & 9 & 8.57 & & 9.4 & 10 & 8.3 & 9.97 & 5 & 8.77 & 10 & 6.33 \\
\hline \multirow[t]{3}{*}{ EO } & 30 & 4 & 4 & 4 & 3.17 & 3 & & 3.8 & 1.9 & 4 & 6.17 & 2.17 & 8.23 & 2 & 3.77 \\
\hline & 100 & 4 & 4 & 4 & 5.87 & 5.9 & & 7.97 & 8.27 & 4 & 7.07 & 2.33 & 8.1 & 2 & 4 \\
\hline & 200 & 4 & 4 & 4 & 7.77 & 8.5 & & 3.47 & 9 & 4 & 6.9 & 3.13 & 8.23 & 2 & 4 \\
\hline \multirow[t]{3}{*}{ HHO } & 30 & 2 & 2 & 2.47 & 2 & 2 & & 2.07 & 3.97 & 2 & 2.57 & 2.83 & 3.27 & 3 & 2 \\
\hline & 100 & 2 & 2 & 2.27 & 2 & 2 & & 2.43 & 2.8 & 2 & 2.37 & 2.8 & 3.07 & 3 & 2 \\
\hline & 200 & 2 & 2 & 2.3 & 2 & 2 & & 2.37 & 2.7 & 2 & 2.5 & 2.1 & 3.4 & 3 & 2 \\
\hline \multirow[t]{3}{*}{ SFO } & 30 & 6 & 6.03 & 6.1 & 3.87 & 4 & & 3.4 & 4.03 & 6.47 & 4.3 & 6.2 & 10 & 5.37 & 6.03 \\
\hline & 100 & 5.9 & 6.1 & 6.1 & 3.03 & 3 & & 3.1 & 2.9 & 6.23 & 4.3 & 6.3 & 10 & 5.47 & 5.97 \\
\hline & 200 & 5 & 5 & 6.03 & 3.07 & 3 & & 3.23 & 3.07 & 5.4 & 4.27 & 6.17 & 10 & 5.53 & 5 \\
\hline \multirow[t]{3}{*}{ WOA } & 30 & 3 & 3 & 2.53 & 10 & 10 & & 9.87 & 9.7 & 3 & 7.73 & 4 & 7.07 & 4 & 3.23 \\
\hline & 100 & 3 & 3 & 2.73 & 10 & 10 & & 9.5 & 8.47 & 3 & 6.4 & 3.87 & 6.93 & 4 & 3 \\
\hline & 200 & 3 & 3 & 2.7 & 10 & 9.93 & & 9.03 & 7.97 & 3 & 6.63 & 3.77 & 6.83 & 4 & 3 \\
\hline Function & Dimensionality & F14 & F15 & F16 & F17 & F18 & F19 & F20 & $\mathrm{F} 21$ & $\mathrm{~F} 22$ & $\mathrm{~F} 23$ & Avg & rank & Overal & ank \\
\hline \multirow[t]{3}{*}{ MGEHO } & 30 & 1 & 5.83 & 4.87 & 1 & 1 & 1 & 1 & 7 & 1 & 1 & 2.21 & & 1 & \\
\hline & 100 & 1 & 4.03 & 4.87 & 1 & 1 & 1 & 1 & 7.53 & 1 & 1 & 2.06 & & 1 & \\
\hline & 200 & 1 & 4.07 & 4.23 & 1 & 1.03 & 1 & 1 & 6.83 & 1 & 1 & 2.09 & & 1 & \\
\hline \multirow[t]{3}{*}{ EHO } & 30 & 8.17 & 4.6 & 4.43 & 7.73 & 8.17 & 8.73 & 8.23 & 7.33 & 6.13 & 5.63 & 6.94 & & 8 & \\
\hline & 100 & 8.43 & 3.6 & 3.67 & 7.3 & 7.93 & 8.7 & 8.37 & 6.97 & 5.57 & 5.77 & 6.6 & & 7 & \\
\hline & 200 & 8.47 & 2.93 & 3.9 & 7.23 & 7.43 & 8 & 8.43 & 7 & 5.7 & 5.53 & 6.43 & & 7 & \\
\hline \multirow[t]{3}{*}{ EEHO15 } & 30 & 7.03 & 4.7 & 4.87 & 6.4 & 6.73 & 6.97 & 7.5 & 10 & 3.7 & 3.9 & 6.11 & & 7 & \\
\hline & 100 & 7.4 & 3.97 & 3.6 & 6.43 & 6.93 & 6.73 & 7.43 & 9.97 & 3.9 & 3.83 & 5.79 & & 6 & \\
\hline & 200 & 7.13 & 3.87 & 3.93 & 6.13 & 6.93 & 6.43 & 7.53 & 10 & 3.77 & 3.87 & 5.72 & & 6 & \\
\hline \multirow[t]{3}{*}{ OEHO } & 30 & 8.17 & 4.8 & 4.63 & 8.33 & 9.4 & 8.87 & 8.43 & 6.33 & 5.17 & 5.37 & 7.15 & & 9 & \\
\hline & 100 & 8.03 & 3.47 & 3.7 & 7.83 & 9.37 & 8.77 & 8.4 & 7.27 & 5.53 & 5.5 & 6.92 & & 8 & \\
\hline & 200 & 7.97 & 3.5 & 3.13 & 7.53 & 9.5 & 8.43 & 8.2 & 7.33 & 5.57 & 5.57 & 6.74 & & 8 & \\
\hline \multirow[t]{3}{*}{ LFEHO } & 30 & 9.5 & 8.4 & 7.3 & 9.33 & 9.1 & 9.23 & 9.83 & 4.33 & 6.83 & 7.67 & 8.25 & & 10 & \\
\hline & 100 & 9.63 & 6 & 6.23 & 9.17 & 9.4 & 9.47 & 9.77 & 4.7 & 7.5 & 7.53 & 7.98 & & 10 & \\
\hline & 200 & 9.53 & 6.03 & 6.73 & 8.93 & 9.33 & 9.47 & 9.8 & 5.23 & 7 & 7.7 & 7.95 & & 10 & \\
\hline
\end{tabular}


Table 12 (continued)

\begin{tabular}{llllllllllllll}
\hline Function & Dimensionality & F14 & F15 & F16 & F17 & F18 & F19 & F20 & F21 & F22 & F23 & Avg. rank & Overall rank \\
\hline IGWO & 30 & 3.8 & 5.03 & 7.57 & 8.1 & 2 & 5 & 5 & 1 & 10 & 9.93 & 6 & 6 \\
& 100 & 5.8 & 10 & 9.53 & 9.1 & 5 & 5.63 & 5 & 1 & 10 & 10 & 7.28 & 9 \\
& 200 & 6.9 & 10 & 9.93 & 9.83 & 5.23 & 7.6 & 6.03 & 1.03 & 10 & 10 & 7.83 & 9 \\
EO & 30 & 2.77 & 1.7 & 5.17 & 3.97 & 3 & 4 & 4 & 3 & 8.2 & 7.87 & 4.3 & 3 \\
& 100 & 2 & 8.6 & 9.47 & 3.97 & 2 & 4 & 4 & 2 & 8.53 & 8.07 & 5.22 & 4 \\
& 200 & 2 & 8.97 & 9.07 & 3.9 & 2.03 & 4 & 4 & 2.03 & 8.7 & 8.57 & 5.4 & 5 \\
HHO & 30 & 3.77 & 4.3 & 3.13 & 2.63 & 4 & 2.93 & 2 & 8 & 2 & 2 & 2.91 & 2 \\
& 100 & 3 & 2.57 & 3 & 2.63 & 3 & 3 & 2 & 7.23 & 2 & 2 & 2.66 & 2 \\
SFO & 200 & 3 & 2.97 & 2.9 & 2.87 & 3.03 & 3 & 2 & 5.97 & 2 & 2 & 2.64 & 2 \\
& 30 & 5.7 & 5.8 & 3.13 & 5.07 & 6.6 & 6.2 & 6 & 6 & 4.6 & 3.3 & 5.4 & 4 \\
WOA & 100 & 5.47 & 4.43 & 2.93 & 5.1 & 6.37 & 5.7 & 6.03 & 5.33 & 3.93 & 3.8 & 5.11 & 3 \\
& 200 & 5 & 4.7 & 3.17 & 5.07 & 6.43 & 5.07 & 5 & 6.5 & 4.13 & 3.83 & 4.99 & 3 \\
& 100 & 5.1 & 9.83 & 9.9 & 2.43 & 5 & 2.07 & 3 & 2 & 7.37 & 8.33 & 5.75 & 5 \\
\end{tabular}

functions cover four categories: F1- F3 and F16-F17 are unimodal functions, F4-F10 and F18 are multimodal functions, F11-F14 and F19-F28 are mixed-state functions, and F29-F32 are compositional functions. It is well known that unimodal functions are suitable for confirming the exploratory ability of the method because there exists only one optimal solution. Unlike unimodal functions, there are a large number of locally optimal solutions in the multimodal case. The number of these solutions increases with dimensionality, which is used to test the capability of the exploitation and jumping out of the local optimum.

\subsubsection{Experimental results and analysis}

The comparison results are shown in Table 18. The table reports the mean, standard deviation obtained for 30 runs. The best solutions for each function are in bold. According to the results, it is clear that MGEHO outperforms the comparison algorithm on most of the functions. Compared to the other metaheuristics, MGEHO obtained the first ranking on 18 functions, which is better than any of the comparison algorithms. Although both MGEHO and EHOI are hybrid GWOs, the former is significantly superior to the latter. The main reason is that MGEHO not only uses a mixture of EHO and MRFO as the information sharing method but also utilizes two other information enhancement methods, which contributes to the performance of the algorithm.

Figure 5 shows the convergence curves of the algorithm on the three functions. Where the horizontal axis represents the number of function iterations and the vertical axis represents the average results obtained by the algorithm in
30 independent runs. It is noted that MGEHO has better accuracy in finding the best results than any of the algorithms, while the convergence rate can be maintained at a faster level. The experiments show that MGEHO has the advantage of handling complex problems.

\subsubsection{Statistical analysis}

The Wilcoxon signed-rank test with a significance level of $5 \%$ was adopted to analyze the comparison results of different algorithms, as shown in Table 20. Where " $+/=/-$ " refers to the number of MGEHO wins on the function, ties on the function, and loses on the function. From observing the results in the table, it can be seen that the number of times MGEHO outperforms other metaheuristics is $31,27,22,29,29,27,32$, respectively. Most of the data in the table are less than the significance level of 0.05 , so it can be determined that MGEHO significantly outperforms one of the other seven algorithms.

Another statistical procedure of Holms was also invoked in this research [52]. Holm's test is a post hoc testing procedure that is typically used to correct for experimental precision in multiple comparisons. MGEHO was chosen as the control algorithm. $p$ value provides information regarding whether the statistical hypothesis test is significant or not, and it also indicates the significance of the results. A smaller $p$ value indicates stronger evidence against the null hypothesis. All algorithms are ranked according to the $p$ value and compared to $a /(k-i)$, where the significance level is $0.05, k$ is the degree of freedom, and $i$ is the algorithm number. The test starts with the most significant $p$ value and rejects the null hypothesis 

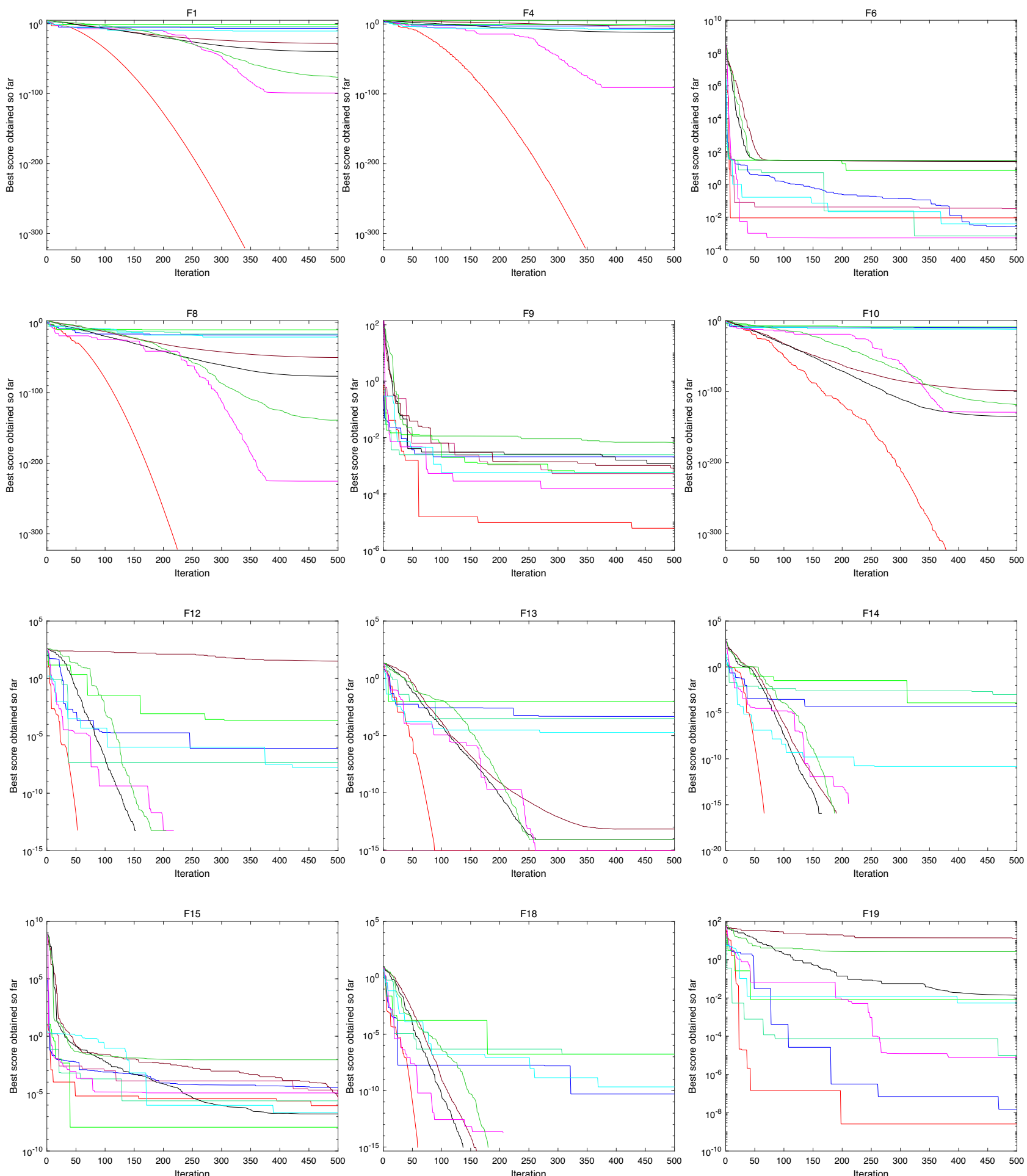

Fig. 3 Convergence curves 

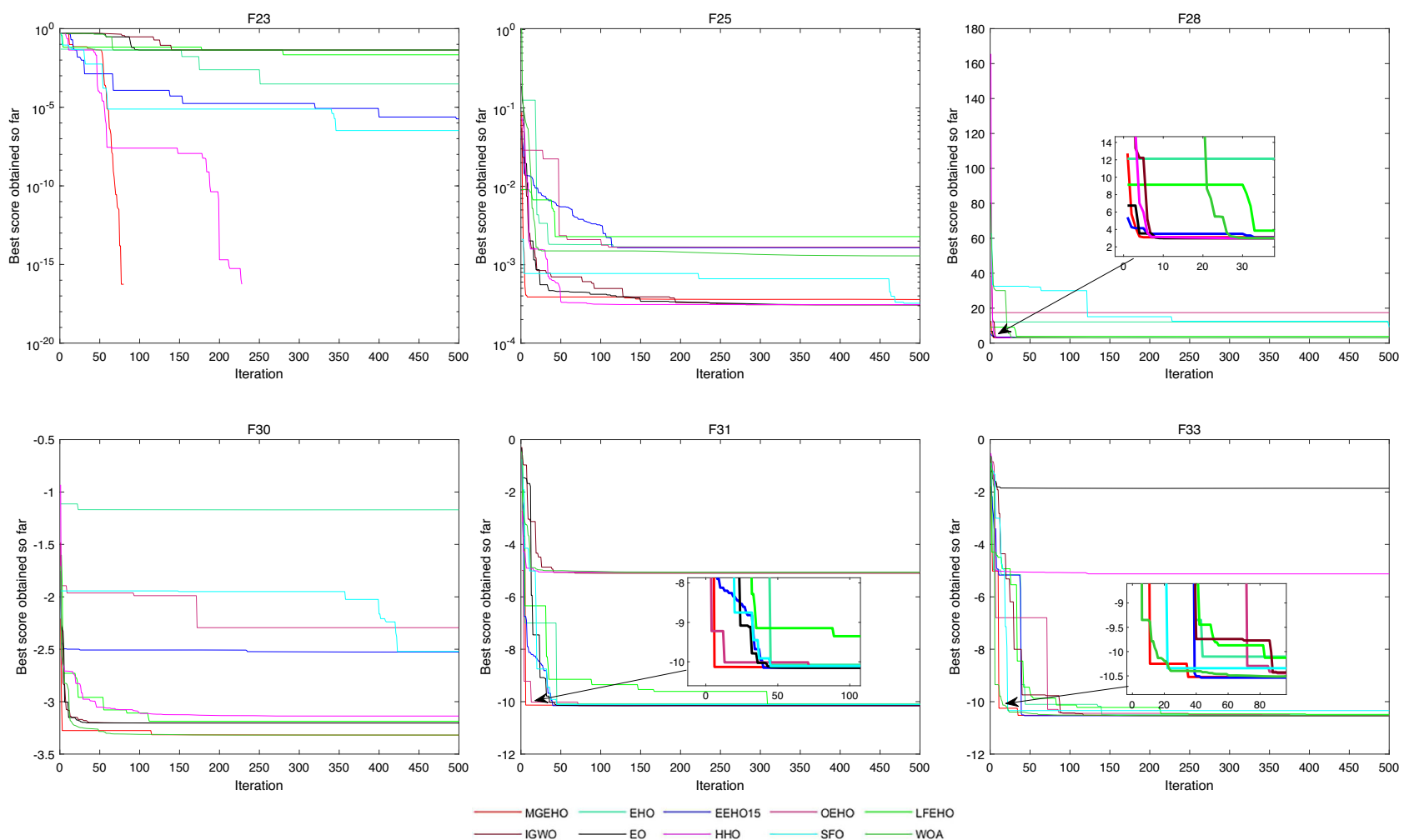

Fig. 3 (continued)

Table 13 Nonparametric statistical comparison based on the Friedman rank test for F24 F33

\begin{tabular}{lllllllllllll}
\hline Function & F24 & F25 & F26 & F27 & F28 & F29 & F30 & F31 & F32 & F33 & Avg. rank & Overall rank \\
\hline MGEHO & 2.13 & 3.57 & 1.53 & 5.2 & 4.87 & 2.97 & 3.87 & 1.83 & 1.2 & 1.73 & 2.89 & 1 \\
EHO & 3 & 8.63 & 9.3 & 9 & 9.33 & 9.37 & 9.4 & 5.7 & 5.43 & 4.77 & 7.39 & 10 \\
EEHO15 & 5.63 & 7.73 & 2.8 & 3.2 & 6.4 & 7.07 & 7.37 & 3.2 & 2.5 & 2.43 & 4.83 & 4 \\
OEHO & 2.6 & 9.07 & 9.13 & 8.7 & 8.67 & 8.87 & 9.33 & 5.13 & 5.4 & 5.5 & 7.24 & 9 \\
LFEHO & 3.4 & 6.13 & 7.7 & 8.7 & 7.93 & 6.1 & 4.7 & 5.57 & 5.67 & 5.8 & 6.17 & 7 \\
IGWO & 7.57 & 1.63 & 4.97 & 4.23 & 1.53 & 4.2 & 1.73 & 1.87 & 2.33 & 1.93 & 3.2 & 2 \\
EO & 8.37 & 3.97 & 5.77 & 5.23 & 1.53 & 4.67 & 2.73 & 3.6 & 3.93 & 4.17 & 4.4 & 3 \\
HHO & 6.37 & 3.7 & 3.43 & 2.9 & 2.93 & 2.37 & 5.6 & 9.8 & 9.73 & 9.7 & 5.65 & 6 \\
SFO & 9.87 & 4.47 & 7.07 & 5.4 & 7.57 & 5.93 & 6.83 & 7.7 & 7.73 & 7.73 & 7.03 & 8 \\
WOA & 6.07 & 6.1 & 3.3 & 2.43 & 4.23 & 3.47 & 3.43 & 7.6 & 8.07 & 8.23 & 5.29 & 5 \\
\hline
\end{tabular}

sequentially, and whenever a null hypothesis cannot be rejected, the test stops and considers all remaining hypotheses accepted. As can be seen in Table 19, the $p$ values of MTDE, EESHHO, HGS, EHOI, SMA, RUN, and GBO are smaller than their $a /(k-i)$ values, which means that the equality hypothesis is rejected and there are significant differences between these five algorithms and the control algorithm.

\subsubsection{Running time analysis}

To analyze the differences between MGEHO and other metaheuristics, the running time of the algorithm on 32 functions is presented in Table 21. The results of time in the table for each algorithm are based on 30 independent runs. As can be seen from the table, SMA takes the most computation time. While MTDE is the fastest in runtime, and this advantage is absolute. The proposed MGEHO ranks six among the compared algorithms but is faster than 

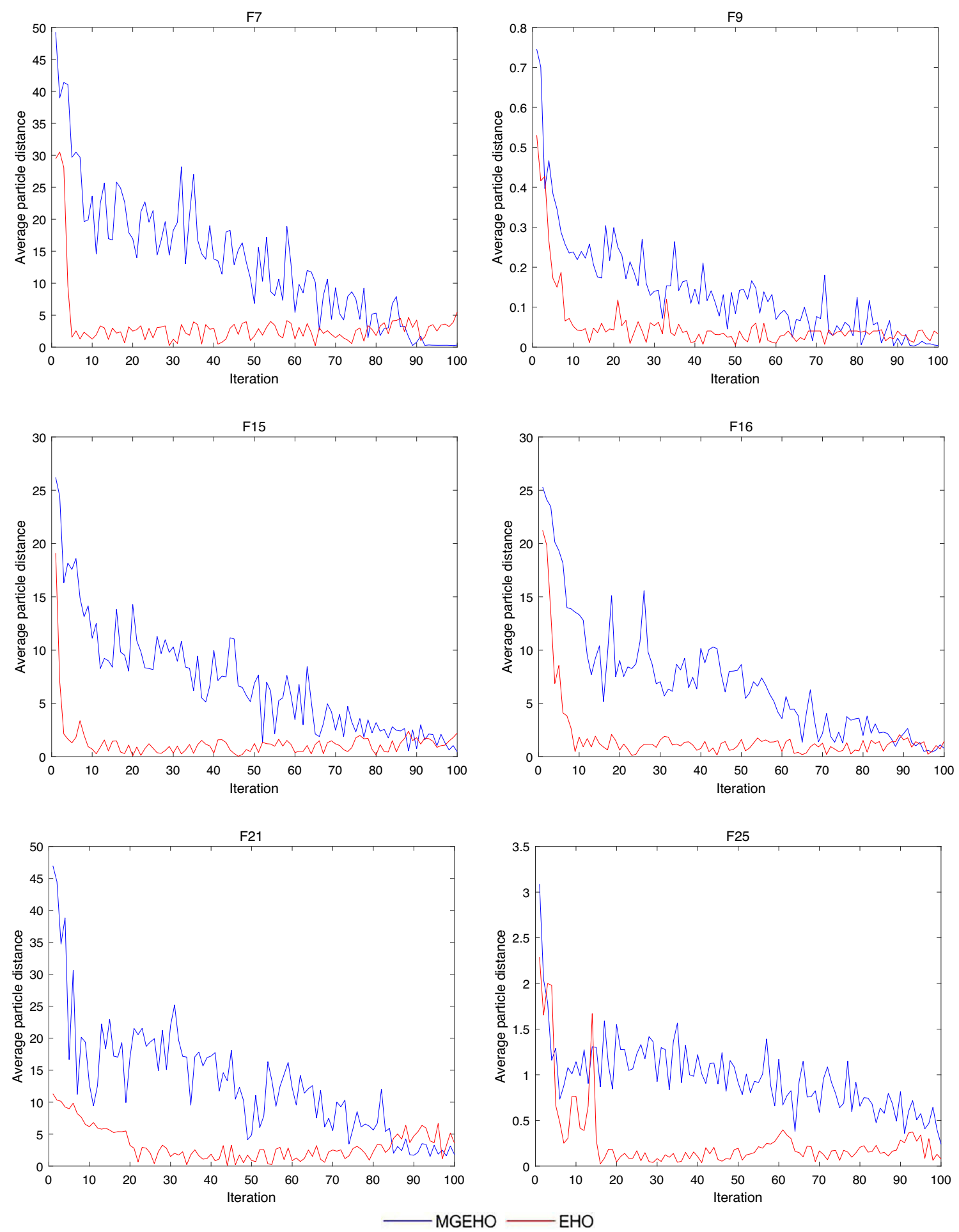

Fig. 4 Diversity curves of MGEHO and EHO

RUN and SMA. The comparison reveals that the embedding of different operators implements MGEHO require more computation time in exchange for performance improvement. But overall, this trade-off is acceptable because their time complexity is in the same order of magnitude. 


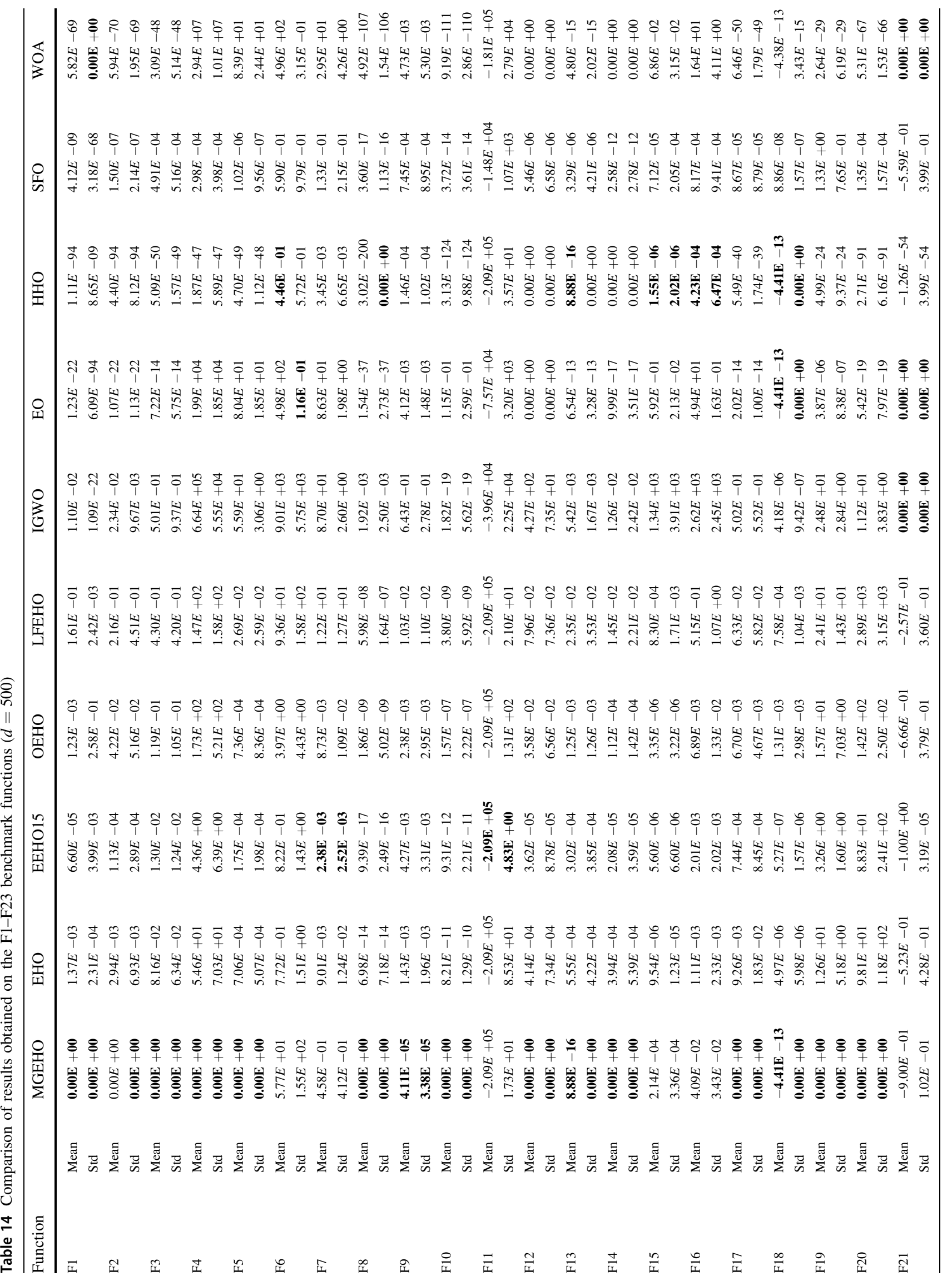




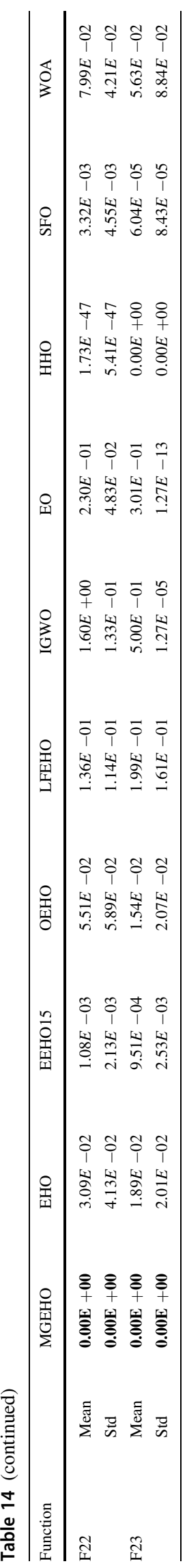

\section{Applications of MGEHO on engineering problems}

There are many cases in real-world engineering that are essentially optimization problems [40]. In this subsection, MGEHO is used to determine solutions to real engineering problems and compared with other algorithms to check the applicability of the proposed algorithm. Three practical engineering problems are selected, namely, a design problem regarding a gear train, a design problem with a three-bar truss, and a design problem with respect to a pressure vessel. To handle optimization constraints, constraint methods need to be integrated into the optimizer. Hybrid methods, repair algorithms, special operators, and penalty functions are applicable [53]. Among them, penalty functions are the simplest and easiest to implement. The death penalty is the most suitable type of penalty function. It can automatically eliminate feasible solutions by constructing the main objective value of a mathematical model and applying heuristic algorithms [54]. Therefore, the three abovementioned mathematical model problems are treated in this way. The population size $N$ is uniformly set to 30 , and the maximum number of iterations $t_{\max }$ is 500 .

\subsection{Design problem of a gear train}

Proposed by Sandgren [55], this problem is a discrete unconstrained optimization problem. Four variables $x_{1}, x_{2}, x_{3}, x_{4}$ are involved, which represent the number of teeth in each of the four gears of the train. All variables are positive integers and lie in the interval $[12,60]$. The main objective of the problem is to minimize the gear ratio cost, and the mathematical model is expressed as follows:

$$
\begin{gathered}
\min \quad F_{1}\left(x_{1}, x_{2}, x_{3}, x_{4}\right)=\left(\frac{1}{6.931}-\frac{x_{2} x_{3}}{x_{1} x_{4}}\right)^{2} \\
\text { where } \quad 12 \leq x_{1}, x_{2}, x_{3}, x_{4} \leq 60
\end{gathered}
$$

MGEHO is applied to the problem and compared with EHO, EEHO15, OEHO, LFEHO, the IGWO, the $\mathrm{EO}$, the $\mathrm{HHO}$, the SFO, and the WOA. The results are presented in Table 22. The best solution is in bold. Compared with other algorithms, MGEHO achieves the best solution. The best cost obtained is $2.3694 E-$ 16 , which is achieved when $x_{1}, x_{2}, x_{3}, x_{4}$ are set to $36.83,12.04,12.26$, and 27.76 , respectively. 


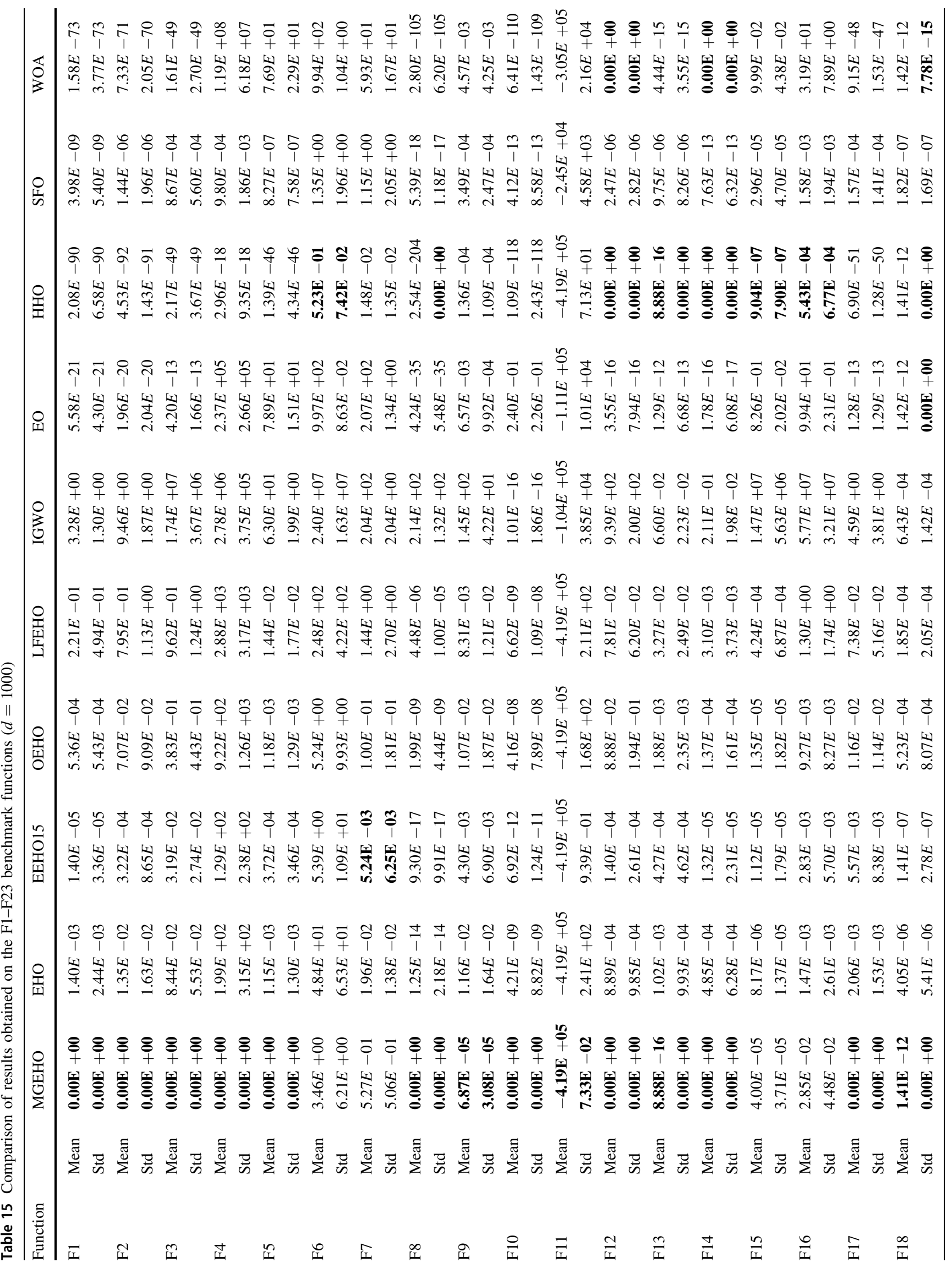




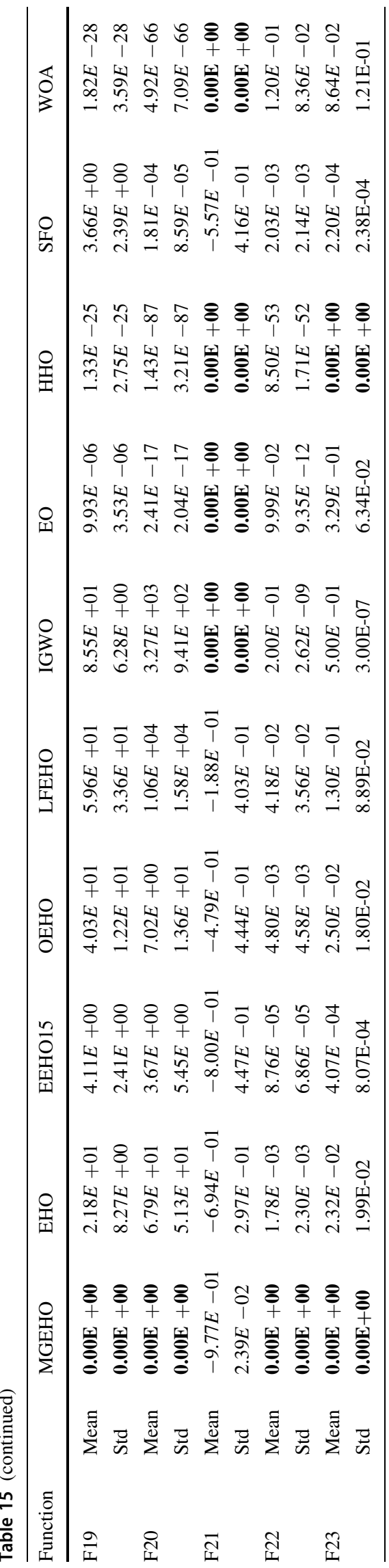

\subsection{Design problem with a three-bar truss}

This problem is a common minimization constraint problem. The objective is to minimize the total weight of three diffeomorphic frames, where the constraints are mainly stress, deflection, and buckling constraints. The structure of the combined diffeomorphic frames is shown in Fig. 6. The mathematical model of this problem is represented as follows :

$$
\begin{aligned}
\min & F_{2}\left(x_{1}, x_{2}\right)=\left(2 \sqrt{2} x_{1}+x_{2}\right) \times L \\
\text { where } \quad & 0 \leq x_{1}, x_{2} \leq 1 \\
\text { s.t. } \quad & \left(\frac{\sqrt{2} x_{1}+x_{2}}{\sqrt{2} x_{1}^{2}+2 x_{1} x_{2}}\right) \times P-\sigma \leq 0 \\
& \left(\frac{x_{2}}{\sqrt{2} x_{1}^{2}+2 x_{1} x_{2}}\right) \times P-\sigma \leq 0 \\
& \left(\frac{1}{x_{1}+\sqrt{2} x_{2}}\right) \times P-\sigma \leq 0 \\
L=100 \mathrm{~cm} \quad & \sigma, P=2 \mathrm{kN} / \mathrm{cm}^{2}
\end{aligned} .
$$

After running MGEHO and EHO, EEHO15, OEHO, LFEHO, the IGWO, the EO, the HHO, the SFO, and the WOA independently 30 times each, the best results are obtained, as shown in Table 23. MGEHO obtains the best solution to the problem in bold (263.89578) when $x_{1}, x_{2}$ are 0.78157 and 0.42853 , respectively. The results show that MGEHO can handle the problem effectively and has superior performance to that of other algorithms.

\subsection{Design problem with respect to a pressure vessel}

This is a common engineering design problem proposed by Kannan and Kramer [56]. Four variables are involved, namely, the thickness of the shell $T_{s}$, the thickness of the head $T_{h}$, the inner radius of the cylinder $R$, and the length of the cylinder $L$. The objective of the problem is to minimize the manufacturing cost of the pressure vessel, as shown in Fig. 7. The problem contains four constraints, three of which are linear, and one is nonlinear. The mathematical form of the problem is as follows: 
Table 16 Results of Friedman rank test of high-dimensional problems

\begin{tabular}{|c|c|c|c|c|c|c|c|c|c|c|c|c|c|c|c|}
\hline Function & Dimensionality & F1 & F2 & F3 & F4 & F5 & F6 & $\mathrm{F}^{7}$ & & F8 & F9 & F10 & F11 & F12 & F13 \\
\hline \multirow[t]{2}{*}{ MGEHO } & 500 & 1.00 & 1.00 & 1.00 & 1.00 & 1.00 & 4.10 & 6. & 20 & 1.00 & 1.30 & 1.00 & 2.20 & 2.50 & 1.55 \\
\hline & 1000 & 1.00 & 1.00 & 1.00 & 1.00 & 1.00 & 3.00 & 5. & 60 & 3 & 5.60 & 1.00 & 2.00 & 1.00 & 1.80 \\
\hline \multirow[t]{2}{*}{ EHO } & 500 & 7.40 & 6.91 & 7.70 & 5.90 & 5.50 & 3.20 & 3. & 20 & 7.90 & 8.20 & 6.90 & 6.50 & 7.00 & 6.40 \\
\hline & 1000 & 7.40 & 7.40 & 7.00 & 4.90 & 5.50 & 3.80 & 2. & 80 & 4.4 & 4.00 & 6.60 & 7.20 & 7.60 & 3.40 \\
\hline \multirow[t]{2}{*}{ EEHO15 } & 500 & 6.50 & 6.36 & 6.20 & 4.70 & 4.30 & 3.10 & 2. & 80 & 7.40 & 4.60 & 7.40 & 4.80 & 6.80 & 7.30 \\
\hline & 1000 & 6.10 & 6.00 & 6.40 & 4.90 & 4.50 & 3.80 & 3. & 40 & 3.8 & 2.80 & 6.20 & 4.80 & 5.80 & 1.80 \\
\hline \multirow[t]{2}{*}{ OEHO } & 500 & 7.37 & 8.09 & 7.60 & 5.10 & 5.20 & 4.50 & 3. & 50 & 8.40 & 5.50 & 9.60 & 4.50 & 8.20 & 7.40 \\
\hline & 1000 & 7.40 & 7.90 & 7.90 & 5.70 & 5.30 & 3.80 & 3. & 40 & 3.8 & 3.40 & 8.20 & 5.60 & 8.40 & 4.40 \\
\hline \multirow[t]{2}{*}{ LFEHO } & 500 & 9.50 & 9.45 & 8.50 & 6.20 & 7.00 & 6.40 & 6. & 50 & 8.20 & 6.60 & 8.70 & 4.30 & 8.70 & 9.60 \\
\hline & 1000 & 9.00 & 8.60 & 8.70 & 6.40 & 6.70 & 6.40 & 5. & 60 & 6.4 & 5.60 & 8.80 & 6.20 & 8.00 & 4.80 \\
\hline \multirow[t]{2}{*}{ IGWO } & 500 & 9.23 & 7.35 & 10.00 & 9.00 & 8.30 & 10.00 & 9. & 60 & 10.00 & 10.00 & 4.50 & 8.90 & 10.00 & 9.30 \\
\hline & 1000 & 10.00 & 10.00 & 10.00 & 9.00 & 8.20 & 10.00 & 9. & 00 & 10 & 9.00 & 10.00 & 10.00 & 4.00 & 8.60 \\
\hline \multirow[t]{2}{*}{ EO } & 500 & 4.00 & 4.00 & 4.00 & 8.00 & 9.20 & 9.00 & 9. & 40 & 4.00 & 7.10 & 6.20 & 8.10 & 2.50 & 4.00 \\
\hline & 1000 & 4.00 & 4.00 & 4.00 & 8.00 & 9.50 & 9.00 & & .00 & 9 & 10.00 & 4.00 & 7.40 & 10.00 & 8.40 \\
\hline \multirow[t]{2}{*}{ HHO } & 500 & 2.00 & 2.00 & 2.30 & 2.00 & 2.00 & 3.10 & 2. & 90 & 2.00 & 2.50 & 2.10 & 3.20 & 2.50 & 1.55 \\
\hline & 1000 & 2.00 & 2.00 & 2.40 & 2.00 & 2.00 & 3.00 & 3. & 20 & 3 & 3.20 & 2.00 & 1.80 & 2.20 & 4.80 \\
\hline \multirow[t]{2}{*}{ SFO } & 500 & 5.00 & 5.00 & 5.00 & 3.10 & 3.00 & 3.70 & 3. & 10 & 5.40 & 4.50 & 5.70 & 10.00 & 5.60 & 5.00 \\
\hline & 1000 & 5.10 & 5.10 & 5.00 & 3.10 & 3.00 & 3.60 & 3. & 40 & 3.6 & 3.40 & 5.20 & 3.20 & 5.20 & 10.00 \\
\hline \multirow[t]{2}{*}{ WOA } & 500 & 3.00 & 3.00 & 2.70 & 10.00 & 9.50 & 7.90 & 7. & 80 & 3.00 & 6.10 & 2.90 & 7.00 & 2.50 & 2.90 \\
\hline & 1000 & 3.00 & 3.00 & 2.60 & 10.00 & 9.30 & 8.00 & & 00 & 8 & 8.00 & 3.00 & 6.80 & 2.80 & 7.00 \\
\hline Function & Dimensionality & F14 & F15 & F16 & F17 & F18 & F19 & F20 & F21 & F22 & F23 & $\begin{array}{l}\text { Avg. } \\
\text { rank }\end{array}$ & & $\begin{array}{l}\text { Overall } \\
\text { rank }\end{array}$ & \\
\hline \multirow[t]{2}{*}{ MGEHO } & 500 & 1.55 & 2.05 & 5.20 & 1.00 & 2.65 & 1.00 & 1.00 & 6.20 & 1.00 & 1.65 & 2.11 & & 1 & \\
\hline & 1000 & 2.40 & 1.70 & 2.00 & 5.80 & 1.70 & 1.00 & 1.00 & 5.00 & 1.00 & 1.60 & 2.25 & & 1 & \\
\hline \multirow[t]{2}{*}{ EHO } & 500 & 7.30 & 7.80 & 2.80 & 7.50 & 7.30 & 7.50 & 8.30 & 7.00 & 5.70 & 6.00 & 6.54 & & 7 & \\
\hline & 1000 & 7.00 & 7.40 & 7.80 & 4.00 & 6.60 & 7.00 & 8.00 & 7.20 & 5.40 & 5.80 & 6.02 & & 6 & \\
\hline \multirow[t]{2}{*}{ EEHO15 } & 500 & 6.40 & 6.40 & 4.40 & 5.70 & 5.90 & 5.80 & 7.00 & 9.90 & 3.60 & 3.90 & 5.79 & & 6 & \\
\hline & 1000 & 6.00 & 6.40 & 6.20 & 4.40 & 5.60 & 5.80 & 6.20 & 8.60 & 4.40 & 3.60 & 5.18 & & 5 & \\
\hline \multirow[t]{2}{*}{ OEHO } & 500 & 7.40 & 7.20 & 3.50 & 7.60 & 9.60 & 8.20 & 7.80 & 7.50 & 6.10 & 5.60 & 6.81 & & 8 & \\
\hline & 1000 & 8.20 & 7.20 & 7.40 & 3.60 & 8.80 & 8.40 & 6.80 & 7.00 & 5.60 & 6.00 & 6.28 & & 7 & \\
\hline \multirow[t]{2}{*}{ LFEHO } & 500 & 9.60 & 9.20 & 5.70 & 9.00 & 9.00 & 8.90 & 9.50 & 5.70 & 7.50 & 8.10 & 7.90 & & 9 & \\
\hline & 1000 & 8.80 & 9.40 & 8.60 & 4.40 & 8.00 & 9.00 & 9.40 & 9.00 & 7.40 & 7.40 & 7.51 & & 9 & \\
\hline \multirow[t]{2}{*}{ IGWO } & 500 & 9.30 & 9.40 & 10.00 & 9.90 & 7.60 & 9.40 & 7.40 & 2.45 & 10.00 & 10.00 & 8.71 & & 10 & \\
\hline & 1000 & 10.00 & 9.60 & 10.00 & 10.00 & 9.60 & 9.60 & 9.60 & 2.70 & 10.00 & 10.00 & 9.04 & & 10 & \\
\hline \multirow[t]{2}{*}{ EO } & 500 & 4.00 & 3.85 & 9.00 & 4.00 & 2.65 & 4.00 & 4.00 & 2.45 & 8.80 & 8.70 & 5.56 & & 5 & \\
\hline & 1000 & 2.80 & 4.00 & 4.00 & 9.00 & 3.70 & 4.00 & 4.00 & 2.70 & 8.40 & 9.00 & 6.36 & & 8 & \\
\hline \multirow[t]{2}{*}{ HHO } & 500 & 1.55 & 2.05 & 2.50 & 2.40 & 2.65 & 3.00 & 2.00 & 2.75 & 2.00 & 1.65 & 2.32 & & 2 & \\
\hline & 1000 & 2.40 & 1.70 & 2.00 & 2.60 & 1.70 & 3.00 & 2.00 & 2.70 & 2.00 & 1.60 & 2.44 & & 2 & \\
\hline \multirow[t]{2}{*}{ SFO } & 500 & 5.00 & 5.00 & 3.30 & 5.30 & 5.60 & 5.20 & 5.00 & 6.60 & 4.10 & 3.90 & 4.96 & & 4 & \\
\hline & 1000 & 5.00 & 5.00 & 5.00 & 3.20 & 6.40 & 5.20 & 5.00 & 7.40 & 3.60 & 3.80 & 4.76 & & 3 & \\
\hline \multirow[t]{2}{*}{ WOA } & 500 & 2.90 & 2.05 & 8.00 & 2.60 & 2.05 & 2.00 & 3.00 & 2.45 & 6.20 & 5.50 & 4.53 & & 3 & \\
\hline & 1000 & 2.40 & 2.60 & 2.00 & 8.00 & 2.90 & 2.00 & 3.00 & 2.70 & 7.20 & 6.20 & 5.10 & & 4 & \\
\hline
\end{tabular}


Table 17 Details of 32 CEC2014 and CEC2017 benchmark functions

Type No. Nescription

CEC 2014 Test functions (Search range: $[-100,100]^{D}$ )

Unimodal functions

$\mathrm{F} 1$

F2

F3

Simple Multimodal functions

ple Mulimodal functions

$\mathrm{F}$
$\mathrm{F} 7$
$\mathrm{~F} 8$
$\mathrm{~F}$

F4

F5

F6

F7

F8

F9

Hybrid functions

Rotated High Conditioned Elliptic Function

100

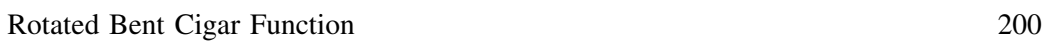

Rotated Discus Function $\quad 300$

Shifted and Rotated Rosenbrock's Function $\quad 400$

Shifted and Rotated Ackley's Function $\quad 500$

Shifted and Rotated Schwefel's Function $\quad 1100$

$\begin{array}{lc}\text { Shifted and Rotated Katsuura Function } & 1200\end{array}$

$\begin{array}{lr}\text { Shifted and Rotated HappyCat Function } & 1300\end{array}$

$\begin{array}{ll}\text { Shifted and Rotated HGBat Function } & 1400\end{array}$

Shifted and Rotated Expanded Griewank's plus Rosenbrock's Function $\quad 1500$

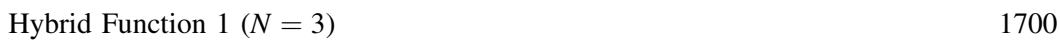

Hybrid Function $2(N=3) \quad 1800$

$\begin{array}{lr}\text { Hybrid Function } 3(N=4) & 1900\end{array}$

$\begin{array}{ll}\text { Hybrid Function } 4(N=4) & 2000\end{array}$

$\begin{array}{ll}\text { Hybrid Function } 5(N=5) & 2100\end{array}$

CEC 2017 Test functions (Search range: $[-100,100]^{D}$ )

Unimodal functions

F16

F17

Simple Multimodal functions

F18

Hybrid functions

F19

F20

F21

F22

F23

F24

F25

F26

F27

F28

Composition functions

F29

F30

F31

F32
Shifted and Rotated Bent Cigar Function

Shifted and Rotated Zakharov Function

100

Shifted and Rotated Rastrigin's Function

300

Hybrid Function $1(N=3)$

500

Hybrid Function $2(N=3)$

1100

Hybrid Function $3(N=3)$

1200

Hybrid Function $4(N=4)$

1300

1400

Hybrid Function $5(N=4)$

1500

Hybrid Function $6(N=4)$

1600

1700

1800

1900

2000

2200

2500

2800

Composition Function $8(N=6)$

Composition Function $10(N=3)$ 
Table 18 Results on CEC2014 and CEC2017 benchmark functions

\begin{tabular}{|c|c|c|c|c|c|c|c|c|c|}
\hline Function & & MGEHO & EHOI & RUN & GBO & HGS & SMA & EESHHO & MTDE \\
\hline \multirow[t]{2}{*}{ F1 } & Mean & $1.34 \mathrm{E}+06$ & $1.88 E+07$ & $2.21 E+06$ & $2.16 E+06$ & $1.26 E+07$ & $8.81 E+06$ & $1.53 E+07$ & $5.82 E+08$ \\
\hline & Std & $7.85 E+05$ & $1.96 E+07$ & $1.15 E+06$ & $1.05 E+06$ & $1.05 E+07$ & $5.57 E+06$ & $9.93 E+06$ & $2.00 E+08$ \\
\hline \multirow[t]{2}{*}{ F2 } & Mean & 3.31E +03 & $1.11 E+07$ & $2.22 E+04$ & $1.09 E+04$ & $4.90 E+05$ & $2.21 E+04$ & $7.22 E+06$ & $1.79 E+10$ \\
\hline & Std & $3.48 \mathrm{E}+03$ & $5.18 E+07$ & $1.06 E+04$ & $9.59 E+03$ & $1.53 E+06$ & $1.22 E+04$ & $1.46 E+07$ & $4.29 E+09$ \\
\hline \multirow[t]{2}{*}{ F3 } & Mean & $1.84 E+03$ & $2.42 E+04$ & $4.89 E+03$ & $2.43 E+03$ & $1.80 E+04$ & $6.52 E+03$ & $9.31 E+03$ & $9.34 E+04$ \\
\hline & Std & $8.90 E+02$ & $1.94 E+04$ & $1.96 E+03$ & $1.67 E+03$ & $1.65 E+04$ & $6.69 E+03$ & $5.62 E+03$ & $1.43 E+04$ \\
\hline \multirow[t]{2}{*}{ F4 } & Mean & $4.74 E+02$ & $5.53 E+02$ & $5.06 E+02$ & $5.15 E+02$ & $5.37 E+02$ & $5.32 E+02$ & $5.43 E+02$ & $2.87 E+03$ \\
\hline & Std & $1.36 \mathrm{E}+01$ & $5.16 E+01$ & $2.90 E+01$ & $4.62 E+01$ & $3.66 E+01$ & $3.61 E+01$ & $4.51 E+01$ & $8.97 E+02$ \\
\hline \multirow[t]{2}{*}{ F5 } & Mean & $5.18 \mathrm{E}+02$ & $5.21 E+02$ & $5.21 E+02$ & $5.21 E+02$ & $5.20 E+02$ & $5.21 E+02$ & $5.20 E+02$ & $5.21 E+02$ \\
\hline & Std & $5.80 \mathrm{E}-02$ & $2.53 E-01$ & $2.36 E-01$ & $2.54 E-01$ & $6.98 E-02$ & $7.05 E-02$ & $7.06 E-02$ & $6.18 E-02$ \\
\hline \multirow[t]{2}{*}{ F6 } & Mean & $4.80 E+03$ & $6.21 E+03$ & $4.44 E+03$ & $5.09 E+03$ & $4.24 E+03$ & $4.46 E+03$ & $4.49 E+03$ & $9.20 E+03$ \\
\hline & Std & $6.37 E+02$ & $8.72 E+02$ & $5.82 E+02$ & $5.79 E+02$ & $4.41 E+02$ & $6.81 E+02$ & $5.20 E+02$ & $4.52 E+02$ \\
\hline \multirow[t]{2}{*}{ F7 } & Mean & $1.20 E+03$ & $1.20 E+03$ & $1.20 E+03$ & $1.20 E+03$ & $1.20 E+03$ & $1.20 E+03$ & $1.20 E+03$ & $1.20 E+03$ \\
\hline & Std & $8.53 E-01$ & $9.06 E-01$ & $9.00 E-01$ & $2.19 E-01$ & $8.44 \mathrm{E}-02$ & $2.27 E-01$ & $9.54 E-02$ & $7.34 E-01$ \\
\hline \multirow[t]{2}{*}{ F8 } & Mean & $1.30 E+03$ & $1.30 E+03$ & $1.30 \mathrm{E}+\mathbf{0 3}$ & $1.30 E+03$ & $1.30 E+03$ & $1.30 E+03$ & $1.30 E+03$ & $1.30 E+03$ \\
\hline & Std & $1.23 E-01$ & $1.41 E-01$ & $8.36 \mathrm{E}-02$ & $1.18 E-01$ & $1.12 E-01$ & $1.03 E-01$ & $1.06 E-01$ & $4.65 E-01$ \\
\hline \multirow[t]{2}{*}{ F9 } & Mean & $1.40 E+03$ & $1.40 E+03$ & $1.40 E+03$ & $1.40 E+03$ & $1.40 E+03$ & $1.40 E+03$ & $1.40 E+03$ & $1.46 E+03$ \\
\hline & Std & $5.10 E-02$ & $1.90 E-01$ & $1.16 E-01$ & $4.90 \mathrm{E}-02$ & $3.58 E-01$ & $3.75 E-01$ & $1.10 E-01$ & $1.29 E+01$ \\
\hline \multirow[t]{2}{*}{ F10 } & Mean & $1.51 E+03$ & $1.59 E+03$ & $1.55 E+03$ & $1.52 E+03$ & $1.52 E+03$ & $1.51 \mathrm{E}+\mathbf{0 3}$ & $1.53 E+03$ & $3.57 E+04$ \\
\hline & Std & $7.19 E+00$ & $4.12 E+01$ & $1.83 E+01$ & $7.34 E+00$ & $5.19 E+00$ & $3.75 \mathrm{E}+00$ & $9.03 E+00$ & $3.24 E+04$ \\
\hline \multirow[t]{2}{*}{ F11 } & Mean & $1.97 E+05$ & $3.30 E+06$ & $1.70 E+05$ & $1.41 \mathrm{E}+05$ & $1.73 E+06$ & $1.74 E+06$ & $1.81 E+06$ & $2.45 E+07$ \\
\hline & Std & $1.57 E+05$ & $2.66 E+06$ & $1.58 E+05$ & $1.16 \mathrm{E}+05$ & $1.20 E+06$ & $1.06 E+06$ & $1.47 E+06$ & $1.07 E+07$ \\
\hline \multirow[t]{2}{*}{ F12 } & Mean & $3.80 E+03$ & $2.39 E+04$ & $3.68 E+03$ & $8.57 E+03$ & $1.27 E+04$ & $2.54 E+04$ & $3.74 E+03$ & $1.57 E+08$ \\
\hline & Std & $1.93 E+03$ & $8.76 E+04$ & $1.54 \mathrm{E}+\mathbf{0 3}$ & $8.49 E+03$ & $9.21 E+03$ & $1.18 E+04$ & $2.77 E+03$ & $9.24 E+07$ \\
\hline \multirow[t]{2}{*}{ F13 } & Mean & $1.92 E+03$ & $1.93 E+03$ & $1.93 E+03$ & $1.92 E+03$ & $1.94 E+03$ & $1.92 E+03$ & $1.92 E+03$ & $2.07 E+03$ \\
\hline & Std & $2.75 E+01$ & $1.52 E+01$ & $3.08 E+01$ & $1.23 \mathrm{E}+01$ & $3.97 E+01$ & $2.30 E+01$ & $2.88 E+01$ & $3.91 E+01$ \\
\hline \multirow[t]{2}{*}{ F14 } & Mean & $8.64 E+03$ & $3.51 E+04$ & $5.26 \mathrm{E}+03$ & $6.24 E+03$ & $3.01 E+04$ & $2.14 E+04$ & $1.68 E+04$ & $7.00 E+04$ \\
\hline & Std & $5.02 E+03$ & $1.61 E+04$ & $2.39 \mathrm{E}+\mathbf{0 3}$ & $3.43 E+03$ & $1.81 E+04$ & $1.64 E+04$ & $5.49 E+03$ & $3.42 E+04$ \\
\hline \multirow[t]{2}{*}{ F15 } & Mean & $8.40 E+04$ & $8.01 E+05$ & $5.69 E+04$ & $6.91 E+04$ & $1.31 E+06$ & $7.64 E+05$ & $6.93 E+05$ & $7.89 E+06$ \\
\hline & Std & $6.98 E+04$ & $8.35 E+05$ & 4.31E +04 & $5.58 E+04$ & $1.03 E+06$ & $5.36 E+05$ & $4.24 E+05$ & $4.98 E+06$ \\
\hline \multirow[t]{2}{*}{ F16 } & Mean & $4.56 E+03$ & $4.89 E+06$ & $1.04 E+04$ & $5.55 E+03$ & $2.24 E+07$ & $1.26 E+04$ & $1.91 E+07$ & $1.40 E+10$ \\
\hline & Std & $3.26 \mathrm{E}+03$ & $9.83 E+06$ & $8.15 E+03$ & $4.35 E+03$ & $4.01 E+07$ & $7.30 E+03$ & $8.00 E+07$ & $3.74 E+09$ \\
\hline \multirow[t]{2}{*}{ F17 } & Mean & $6.45 E+03$ & $4.85 E+04$ & $1.71 \mathrm{E}+\mathbf{0 3}$ & $6.53 E+03$ & $2.73 E+04$ & $5.01 E+03$ & $3.77 E+04$ & $1.57 E+05$ \\
\hline & Std & $3.68 E+03$ & $9.08 E+03$ & $9.60 E+02$ & $3.03 E+03$ & $1.10 E+04$ & $3.08 E+03$ & $6.78 E+03$ & $4.39 E+04$ \\
\hline \multirow[t]{2}{*}{ F18 } & Mean & $6.97 E+02$ & $6.73 E+02$ & $7.05 E+02$ & $6.62 E+02$ & $6.45 E+02$ & $6.16 \mathrm{E}+02$ & $7.10 E+02$ & $8.05 E+02$ \\
\hline & Std & $4.75 E+01$ & $2.98 E+01$ & $4.31 E+01$ & $4.14 E+01$ & $2.65 E+01$ & $2.21 \mathrm{E}+01$ & $4.01 E+01$ & $2.40 E+01$ \\
\hline \multirow[t]{2}{*}{ F19 } & Mean & $1.20 \mathrm{E}+\mathbf{0 3}$ & $1.33 E+03$ & $1.20 E+03$ & $1.26 E+03$ & $1.25 E+03$ & $1.28 E+03$ & $1.24 E+03$ & $5.36 E+03$ \\
\hline & Std & $1.88 \mathrm{E}+01$ & $8.56 E+01$ & $3.33 E+01$ & $7.98 E+01$ & $7.56 E+01$ & $5.41 E+01$ & $4.05 E+01$ & $1.39 E+03$ \\
\hline \multirow[t]{2}{*}{ F20 } & Mean & $2.06 E+05$ & $5.44 E+06$ & $3.91 E+06$ & $3.28 E+05$ & $3.89 E+06$ & $4.07 E+06$ & $3.15 E+06$ & $1.33 E+09$ \\
\hline & Std & $2.04 E+05$ & $7.45 E+06$ & $2.28 E+06$ & $3.55 E+05$ & $2.08 E+06$ & $2.24 E+06$ & $1.70 E+06$ & $5.67 E+08$ \\
\hline F21 & Mean & $1.36 E+04$ & $7.10 E+05$ & $3.05 E+04$ & $1.92 E+04$ & $4.24 E+04$ & $4.06 E+04$ & $1.83 E+04$ & $3.42 E+08$ \\
\hline & Std & $1.18 E+04$ & $1.49 E+06$ & $1.48 E+04$ & $2.12 E+04$ & $2.90 E+04$ & $2.42 E+04$ & $1.87 E+04$ & $1.88 E+08$ \\
\hline F22 & Mean & $8.98 E+03$ & $2.78 E+05$ & $8.39 E+03$ & $6.22 E+03$ & $2.22 E+05$ & $1.35 E+05$ & $3.30 E+05$ & $1.12 E+06$ \\
\hline & Std & $1.32 E+04$ & $3.40 E+05$ & $1.01 E+04$ & $6.05 E+03$ & $2.28 E+05$ & $8.99 E+04$ & $2.21 E+05$ & $8.38 E+05$ \\
\hline F23 & Mean & $7.20 E+03$ & $2.87 E+04$ & $1.55 E+04$ & $9.98 E+03$ & $1.42 E+04$ & $2.19 E+04$ & $5.35 E+03$ & $7.22 E+07$ \\
\hline & Std & $8.84 E+03$ & $2.97 E+04$ & $2.07 E+03$ & $9.96 E+03$ & $1.30 E+04$ & $1.43 E+04$ & $1.72 E+03$ & $4.92 E+07$ \\
\hline F24 & Mean & $2.72 E+03$ & $3.00 E+03$ & $2.82 E+03$ & $2.62 E+03$ & $2.92 E+03$ & $2.54 E+03$ & $2.87 E+03$ & $4.26 E+03$ \\
\hline & Std & $2.77 E+02$ & $4.17 E+02$ & $3.13 E+02$ & $3.16 E+02$ & $2.85 E+02$ & $2.36 \mathrm{E}+02$ & $3.23 E+02$ & $2.97 E+02$ \\
\hline
\end{tabular}


Table 18 (continued)

\begin{tabular}{llllllllll}
\hline Function & & MGEHO & EHOI & RUN & GBO & HGS & SMA & EESHHO & MTDE \\
\hline F25 & Mean & $\mathbf{2 . 2 0 E}+\mathbf{0 3}$ & $2.44 E+03$ & $2.24 E+03$ & $2.34 E+03$ & $2.37 E+03$ & $2.35 E+03$ & $2.26 E+03$ & $2.94 E+03$ \\
& Std & $\mathbf{1 . 8 7 E}+\mathbf{0 2}$ & $2.38 E+02$ & $2.18 E+02$ & $2.17 E+02$ & $2.34 E+02$ & $2.19 E+02$ & $2.40 E+02$ & $1.88 E+02$ \\
F26 & Mean & $2.04 E+05$ & $2.60 E+06$ & $\mathbf{8 . 4 1 E}+\mathbf{0 4}$ & $1.19 E+05$ & $2.54 E+06$ & $1.54 E+06$ & $8.67 E+05$ & $1.59 E+07$ \\
& Std & $1.76 E+05$ & $2.78 E+06$ & $\mathbf{3 . 8 9 E}+\mathbf{0 4}$ & $9.70 E+04$ & $3.42 E+06$ & $1.65 E+06$ & $9.13 E+05$ & $8.38 E+06$ \\
F27 & Mean & $8.10 E+03$ & $2.00 E+05$ & $1.03 E+04$ & $8.54 E+03$ & $2.53 E+04$ & $2.73 E+04$ & $\mathbf{5 . 4 7 E}+\mathbf{0 3}$ & $9.19 E+07$ \\
& Std & $6.78 E+03$ & $6.43 E+05$ & $1.12 E+04$ & $1.04 E+04$ & $2.21 E+04$ & $2.11 E+04$ & $\mathbf{3 . 6 5 E}+\mathbf{0 3}$ & $8.42 E+07$ \\
F28 & Mean & $2.57 E+03$ & $2.64 E+03$ & $\mathbf{2 . 4 8 E}+\mathbf{0 3}$ & $2.57 E+03$ & $2.55 E+03$ & $2.61 E+03$ & $2.56 E+03$ & $3.22 E+03$ \\
& Std & $1.56 E+02$ & $2.18 E+02$ & $\mathbf{1 . 1 5 E}+\mathbf{0 2}$ & $1.48 E+02$ & $2.43 E+02$ & $1.64 E+02$ & $1.74 E+02$ & $2.16 E+02$ \\
F29 & Mean & $\mathbf{3 . 0 3 E}+\mathbf{0 3}$ & $6.65 E+03$ & $3.45 E+03$ & $3.75 E+03$ & $5.20 E+03$ & $5.91 E+03$ & $3.95 E+03$ & $9.62 E+03$ \\
& Std & $\mathbf{7 . 0 5 E}+\mathbf{0 2}$ & $2.62 E+03$ & $1.84 E+03$ & $2.11 E+03$ & $1.74 E+03$ & $8.30 E+02$ & $1.93 E+03$ & $1.86 E+03$ \\
F30 & Mean & $2.90 E+03$ & $2.93 E+03$ & $2.91 E+03$ & $2.90 E+03$ & $2.90 E+03$ & $\mathbf{2 . 8 9 E}+\mathbf{0 3}$ & $2.93 E+03$ & $3.78 E+03$ \\
& Std & $1.99 E+01$ & $2.49 E+01$ & $1.86 E+01$ & $1.83 E+01$ & $1.31 E+01$ & $\mathbf{1 . 2 8 E}+\mathbf{0 1}$ & $2.33 E+01$ & $1.60 E+02$ \\
F31 & Mean & $\mathbf{3 . 2 0 E}+\mathbf{0 3}$ & $3.30 E+03$ & $3.23 E+03$ & $3.23 E+03$ & $3.28 E+03$ & $3.26 E+03$ & $3.28 E+03$ & $4.92 E+03$ \\
& Std & $\mathbf{1 . 0 3 E}+\mathbf{0 1}$ & $1.34 E+01$ & $2.27 E+01$ & $2.28 E+01$ & $5.74 E+01$ & $4.51 E+01$ & $2.35 E+01$ & $4.20 E+02$ \\
F32 & Mean & $\mathbf{8 . 6 7 E}+\mathbf{0 3}$ & $4.29 E+05$ & $3.00 E+05$ & $1.06 E+04$ & $1.39 E+05$ & $4.96 E+04$ & $9.18 E+04$ & $7.03 E+07$ \\
& Std & $\mathbf{2 . 1 5 E}+\mathbf{0 3}$ & $7.22 E+05$ & $3.39 E+05$ & $4.49 E+03$ & $1.70 E+05$ & $2.86 E+04$ & $9.25 E+04$ & $3.53 E+07$ \\
\hline
\end{tabular}
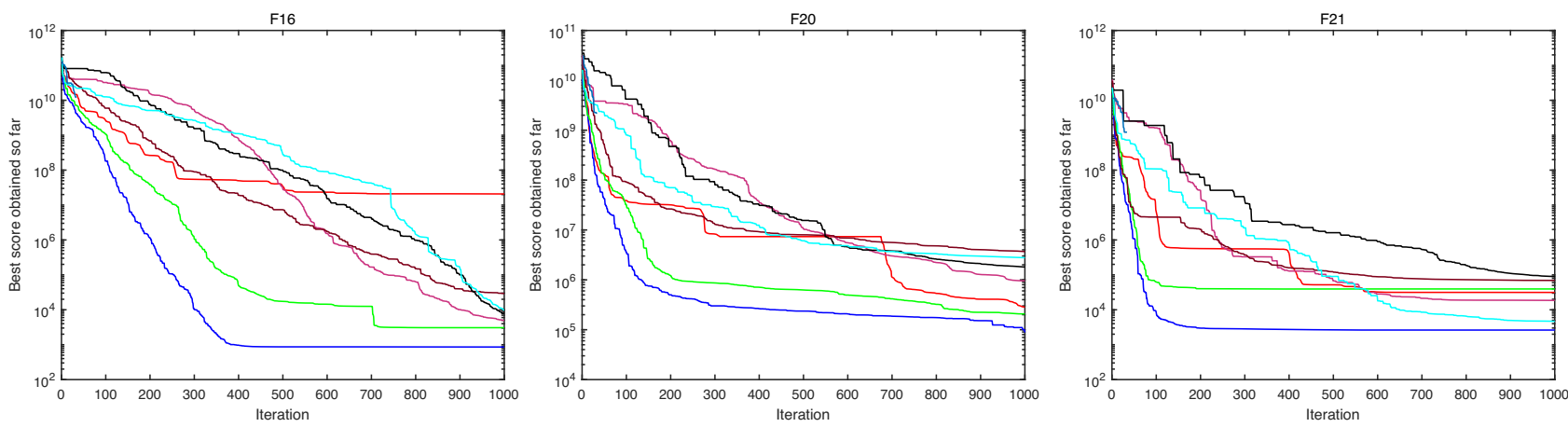

- $\mathrm{EHOI}$ - MGEHO - RUN - GBO - HGS - SMA - EESHHO - MTDE

Fig. 5 Convergence curves of MGEHO and advanced MAs

$$
\begin{aligned}
\min \quad F_{4}(x)= & 0.6224 x_{1} x_{3} x_{4}+1.7781 x_{2} x_{3}^{2} \\
+ & 19.84 x_{1}^{2} x_{3}+3.1661 x_{1}^{2} x_{4} \\
\text { where } & x=\left[x_{1}, x_{2}, x_{3}, x_{4}\right]=\left[T_{s}, T_{h}, R, L\right] \\
\text { s.t. } \quad & g_{1}(x)=-x_{1}+0.0193 x_{3} \leq 0 \\
& g_{2}(x)=-x_{2}+0.00954 x_{3} \leq 0 \\
& g_{3}(x)=-\frac{4}{3} \pi x_{3}^{3}-\pi x_{3}^{2} x_{4}+1296000 \leq 0 \\
& g_{4}(x)=x_{4}-240 \leq 0 \\
& 1 \times 0.0625 \leq x_{1}, x_{2} \leq 99 \times 0.0625 \\
& 10 \leq x_{3}, x_{4} \leq 200
\end{aligned}
$$

OEHO, LFEHO, the IGWO, the EO, the HHO, the SFO, and the WOA, respectively. The obtained experimental results are shown in Table 24. The best solution is in bold. According to the data, MGEHO achieves the best solution among those output by the 10 algorithms, with 0.79386 , $0.38794,40.63038,195.71868$ as the four variable values and 5966.37963 as the best cost.

MGEHO is applied to optimize the problem and compared with nine other algorithms. They are EHO, EEHO15, 
Table 19 Consequence of Holm's test (MGEHO is the control algorithm)

\begin{tabular}{|c|c|c|c|c|c|}
\hline Functions & $p$ value & Rank & MGEHO vs. & $a /(k-i)$ & Significant differences? \\
\hline \multirow[t]{7}{*}{ Unimodal functions } & $5.36 E-23$ & 7.8455 & MTDE & 0.007143 & Yes \\
\hline & $3.63 E-10$ & 5.7273 & EESHHO & 0.008333 & Yes \\
\hline & $9.14 E-08$ & 4.9818 & HGS & 0.01 & Yes \\
\hline & $1.20 E-07$ & 4.7000 & EHOI & 0.0125 & Yes \\
\hline & $8.01 E-07$ & 4.3727 & SMA & 0.016667 & Yes \\
\hline & $8.41 E-05$ & 3.1455 & RUN & 0.025 & Yes \\
\hline & $9.69 E-03$ & 2.4909 & GBO & 0.05 & Yes \\
\hline \multirow[t]{7}{*}{ Simple Multimodal functions } & $1.25 E-10$ & 7.9455 & MTDE & 0.007143 & Yes \\
\hline & $1.91 E-08$ & 5.4364 & EHOI & 0.008333 & Yes \\
\hline & $8.79 E-06$ & 4.2227 & RUN & 0.01 & Yes \\
\hline & $5.42 E-05$ & 3.5773 & GBO & 0.0125 & Yes \\
\hline & $7.56 E-05$ & 3.5818 & HGS & 0.016667 & Yes \\
\hline & $8.23 E-05$ & 3.9364 & SMA & 0.025 & Yes \\
\hline & $9.60 E-04$ & 3.3500 & EESHHO & 0.05 & Yes \\
\hline \multirow[t]{7}{*}{ Hybrid functions } & $9.29 E-24$ & 7.8880 & MTDE & 0.007143 & Yes \\
\hline & $3.14 E-07$ & 4.9960 & SMA & 0.008333 & Yes \\
\hline & $8.34 E-07$ & 5.0080 & HGS & 0.01 & Yes \\
\hline & $4.14 E-06$ & 4.4200 & EHOI & 0.0125 & Yes \\
\hline & $2.93 E-05$ & 4.1160 & EESHHO & 0.016667 & Yes \\
\hline & $1.53 E-03$ & 3.2000 & RUN & 0.025 & Yes \\
\hline & $2.72 E-02$ & 2.7200 & GBO & 0.05 & Yes \\
\hline \multirow[t]{7}{*}{ Composition functions } & $3.49 E-07$ & 7.9750 & MTDE & 0.007143 & Yes \\
\hline & $3.60 E-05$ & 5.5750 & EHOI & 0.008333 & Yes \\
\hline & $1.32 E-04$ & 4.0500 & HGS & 0.01 & Yes \\
\hline & $5.34 E-04$ & 4.4250 & SMA & 0.0125 & Yes \\
\hline & $2.79 E-03$ & 3.2750 & GBO & 0.016667 & Yes \\
\hline & $5.99 E-03$ & 4.4250 & EESHHO & 0.025 & Yes \\
\hline & 3.63E-02 & 3.9250 & RUN & 0.05 & Yes \\
\hline
\end{tabular}

\section{Conclusion}

In this paper, a new hybrid metaheuristic algorithm is proposed, named MGEHO. MGEHO is adapted to address the weaknesses of the original EHO algorithm, such as its low accuracy and poor convergence rate. The clan updating operator in EHO is optimized by incorporating the somersault foraging strategy from MRFO, and the patriarch position updating method is optimized. On this basis, the ability of MGEHO to jump out of local optima and achieve global optimization is effectively improved. Furthermore, a nonlinear convergence factor, which can vary with the operating state, is set. This strategy contributes to coordinating global searches and achieving a balance between exploration and exploitation. To improve the diversity of the given population, Gaussian mutation is also introduced 
Table 20 The $p$ values of the Wilcoxon signed rank test obtained on 32 CEC benchmark functions

\begin{tabular}{|c|c|c|c|c|c|c|c|}
\hline Function & EHOI & RUN & GBO & HGS & SMA & EESHHO & MTDE \\
\hline F1 & $8.15 E-11$ & $3.03 E-06$ & $9.00 E-01$ & $4.08 E-11$ & $6.07 E-11$ & $3.02 E-11$ & $3.02 E-11$ \\
\hline $\mathrm{F} 2$ & $3.47 E-10$ & $3.02 E-11$ & $2.27 E-05$ & $6.12 E-10$ & $1.21 E-10$ & $3.02 E-11$ & $3.02 E-11$ \\
\hline F3 & $1.70 E-08$ & $1.03 E-05$ & $4.64 E-05$ & $2.57 E-07$ & $1.30 E-03$ & $1.47 E-07$ & $3.02 E-11$ \\
\hline F4 & $6.28 E-06$ & $1.37 E-07$ & $7.28 E-05$ & $8.12 E-04$ & $1.17 E-04$ & $2.13 E-05$ & $3.02 E-11$ \\
\hline F5 & $1.44 E-04$ & $1.33 E-07$ & $3.50 E-07$ & $3.02 E-11$ & $3.18 E-05$ & $3.02 E-11$ & $1.17 E-09$ \\
\hline F6 & $3.59 E-05$ & $3.26 E-05$ & $5.75 E-02$ & $5.83 E-03$ & $4.04 E-01$ & $5.40 E-01$ & $3.02 E-11$ \\
\hline F7 & $2.06 E-05$ & $2.12 E-08$ & $1.07 E-09$ & $3.02 E-11$ & $5.49 E-11$ & $3.02 E-11$ & $2.92 E-09$ \\
\hline F8 & $8.68 E-03$ & $1.17 E-04$ & $1.87 E-05$ & $1.25 E-05$ & $1.12 E-02$ & $9.07 E-03$ & $3.02 E-11$ \\
\hline F9 & $6.74 E-06$ & $3.63 E-06$ & $7.28 E-05$ & $1.11 E-06$ & $1.68 E-03$ & $5.30 E-04$ & $3.02 E-11$ \\
\hline F10 & $3.02 E-11$ & $8.99 E-11$ & $4.71 E-04$ & $1.99 E-04$ & $1.50 E-04$ & $1.56 E-08$ & $3.02 E-11$ \\
\hline F11 & $8.10 E-10$ & $7.06 E-04$ & $3.51 E-03$ & $2.67 E-09$ & $4.98 E-11$ & $2.37 E-10$ & $3.02 E-11$ \\
\hline F12 & $3.34 E-03$ & $8.30 E-03$ & $7.24 E-04$ & $1.78 E-04$ & $1.33 E-10$ & $3.63 E-01$ & $3.02 E-11$ \\
\hline F13 & $9.83 E-08$ & $6.01 E-08$ & $1.76 E-02$ & $1.11 E-04$ & $6.97 E-03$ & $8.66 E-05$ & $7.39 E-11$ \\
\hline F14 & $8.99 E-11$ & $5.87 E-04$ & $1.91 E-03$ & $9.06 E-08$ & $1.17 E-04$ & $3.52 E-07$ & $3.02 E-11$ \\
\hline F15 & $1.69 E-09$ & $2.06 E-07$ & $3.40 E-08$ & $8.89 E-10$ & $3.20 E-09$ & $2.61 E-10$ & $3.02 E-11$ \\
\hline F16 & $3.30 E-04$ & $2.83 E-04$ & $3.85 E-03$ & $2.46 E-04$ & $4.40 E-04$ & $3.30 E-04$ & $1.83 E-04$ \\
\hline F17 & $1.83 E-04$ & $5.87 E-02$ & $6.23 E-01$ & $3.30 E-04$ & $2.12 E-01$ & $1.83 E-04$ & $1.83 E-04$ \\
\hline F18 & $6.23 E-02$ & $3.45 E-04$ & $5.21 E-01$ & $5.21 E-01$ & $5.83 E-04$ & $9.11 E-03$ & $1.83 E-04$ \\
\hline F19 & $2.83 E-06$ & $7.34 E-04$ & $3.07 E-04$ & $1.21 E-04$ & $7.28 E-03$ & $4.51 E-04$ & $1.83 E-04$ \\
\hline $\mathrm{F} 20$ & $4.40 E-04$ & $3.30 E-04$ & $1.13 E-02$ & $2.46 E-04$ & $4.40 E-04$ & $2.46 E-04$ & $1.83 E-04$ \\
\hline F21 & $5.80 E-06$ & $2.41 E-04$ & $5.71 E-04$ & $8.90 E-04$ & $2.57 E-04$ & $6.23 E-04$ & $1.83 E-04$ \\
\hline $\mathrm{F} 22$ & $1.83 E-04$ & $2.41 E-01$ & $4.27 E-01$ & $1.83 E-04$ & $2.46 E-04$ & $2.46 E-04$ & $1.83 E-04$ \\
\hline F23 & $5.39 E-05$ & $2.83 E-04$ & $1.04 E-04$ & $1.73 E-02$ & $5.80 E-03$ & $7.34 E-05$ & $1.83 E-04$ \\
\hline F24 & $1.04 E-04$ & $5.71 E-04$ & $6.78 E-02$ & $8.13 E-04$ & $2.70 E-01$ & $4.73 E-05$ & $1.83 E-04$ \\
\hline F25 & $1.40 E-08$ & $9.70 E-06$ & $3.07 E-07$ & $8.50 E-07$ & $6.78 E-07$ & $2.73 E-09$ & $2.46 E-04$ \\
\hline F26 & $2.46 E-04$ & $1.40 E-02$ & $1.04 E-01$ & $4.40 E-04$ & $3.30 E-04$ & $3.76 E-02$ & $1.83 E-04$ \\
\hline F27 & $2.57 E-05$ & $4.25 E-02$ & $1.86 E-01$ & $1.04 E-03$ & $8.40 E-04$ & $5.39 E-02$ & $1.83 E-04$ \\
\hline F28 & $2.73 E-05$ & $2.11 E-02$ & $7.34 E-04$ & $1.86 E-01$ & $9.70 E-05$ & $9.70 E-01$ & $1.83 E-04$ \\
\hline F29 & $3.81 E-07$ & $6.47 E-06$ & $2.06 E-07$ & $2.01 E-06$ & $1.25 E-07$ & $1.68 E-08$ & $2.61 E-10$ \\
\hline F30 & $6.95 E-07$ & $1.08 E-07$ & $3.01 E-04$ & $1.19 E-06$ & $5.09 E-08$ & $3.51 E-08$ & $3.02 E-11$ \\
\hline F31 & $3.02 E-11$ & $9.12 E-09$ & $5.76 E-09$ & $1.17 E-07$ & $3.03 E-10$ & $5.00 E-09$ & $3.02 E-11$ \\
\hline F32 & $4.20 E-10$ & $3.02 E-11$ & $2.06 E-11$ & $4.08 E-11$ & $3.02 E-11$ & $2.61 E-10$ & $3.02 E-11$ \\
\hline$+/=/-$ & $31 / 0 / 1$ & $27 / 3 / 2$ & $22 / 2 / 8$ & $29 / 1 / 2$ & $29 / 0 / 3$ & $27 / 1 / 4$ & $32 / 0 / 0$ \\
\hline
\end{tabular}

to $\mathrm{EHO}$ to adjust the position of the worst individual in each clan. In addition, MGEHO is tested on 33 classical benchmark functions and compared with EHO, EEHO15, OEHO, LFEHO, the IGWO, the EO, HHO, the SFO, and the WOA. The experiment on 32 modern CEC2014 and CEC2017 benchmark functions is also performed among MGEHO, EHOI, RUN, GBO, HGS, SMA, EESHHO, and MTDE. The results show that MGEHO is more effective 
Table 21 Time comparison between MGEHO and other selected algorithms on 32 benchmark functions

\begin{tabular}{|c|c|c|c|c|c|c|c|c|}
\hline Functions & MGEHO & EHOI & RUN & GBO & HGS & SMA & EESHHO & MTDE \\
\hline F1 & 2.32 & 1.30 & 2.80 & 1.63 & 0.39 & 3.49 & 0.75 & 0.06 \\
\hline $\mathrm{F} 2$ & 2.31 & 1.05 & 2.42 & 1.44 & 0.27 & 3.20 & 0.63 & 0.04 \\
\hline F3 & 1.80 & 0.97 & 2.21 & 1.37 & 0.25 & 2.94 & 0.61 & 0.04 \\
\hline $\mathrm{F} 4$ & 1.73 & 0.77 & 1.67 & 0.95 & 0.20 & 2.29 & 0.47 & 0.03 \\
\hline F5 & 1.13 & 0.52 & 1.30 & 0.65 & 0.15 & 1.43 & 0.30 & 0.02 \\
\hline F6 & 3.19 & 1.59 & 3.32 & 1.94 & 0.53 & 3.97 & 0.90 & 0.08 \\
\hline F7 & 2.90 & 2.39 & 3.61 & 2.07 & 2.65 & 3.83 & 1.33 & 0.08 \\
\hline F8 & 2.49 & 1.13 & 2.56 & 1.51 & 0.33 & 3.31 & 0.68 & 0.04 \\
\hline F9 & 2.56 & 1.13 & 2.67 & 1.50 & 0.32 & 3.36 & 0.70 & 0.04 \\
\hline F10 & 2.21 & 1.13 & 2.48 & 1.46 & 0.33 & 3.18 & 0.64 & 0.04 \\
\hline F11 & 2.09 & 0.92 & 1.93 & 1.18 & 0.28 & 2.42 & 0.53 & 0.04 \\
\hline F12 & 1.35 & 0.59 & 1.29 & 0.75 & 0.17 & 1.62 & 0.34 & 0.02 \\
\hline F13 & 1.74 & 1.35 & 1.95 & 1.09 & 0.51 & 2.03 & 0.70 & 0.03 \\
\hline F14 & 1.27 & 0.57 & 1.26 & 0.75 & 0.16 & 1.59 & 0.34 & 0.02 \\
\hline F15 & 1.41 & 0.64 & 1.27 & 0.75 & 0.18 & 1.67 & 0.37 & 0.02 \\
\hline F16 & 1.33 & 0.59 & 1.44 & 0.94 & 0.23 & 1.75 & 0.37 & 0.04 \\
\hline F17 & 1.27 & 0.53 & 1.20 & 0.68 & 0.15 & 1.56 & 0.31 & 0.03 \\
\hline F18 & 1.39 & 0.58 & 1.20 & 0.71 & 0.19 & 1.61 & 0.34 & 0.02 \\
\hline F19 & 1.13 & 0.55 & 1.18 & 0.73 & 0.17 & 1.53 & 0.34 & 0.03 \\
\hline F20 & 1.40 & 0.60 & 1.22 & 0.72 & 0.18 & 1.56 & 0.34 & 0.02 \\
\hline F21 & 1.44 & 0.62 & 1.30 & 0.77 & 0.18 & 1.66 & 0.35 & 0.02 \\
\hline F22 & 1.59 & 0.65 & 1.28 & 0.76 & 0.21 & 1.62 & 0.41 & 0.02 \\
\hline F23 & 1.63 & 0.71 & 1.52 & 0.89 & 0.19 & 2.00 & 0.42 & 0.02 \\
\hline F24 & 1.39 & 0.59 & 1.24 & 0.73 & 0.19 & 1.58 & 0.35 & 0.02 \\
\hline F25 & 1.53 & 0.81 & 1.70 & 0.87 & 0.28 & 1.73 & 0.46 & 0.02 \\
\hline F26 & 1.28 & 0.59 & 1.22 & 0.70 & 0.17 & 1.56 & 0.34 & 0.02 \\
\hline F27 & 1.53 & 1.80 & 2.19 & 1.32 & 0.76 & 2.13 & 0.95 & 0.04 \\
\hline F28 & 2.02 & 0.87 & 1.84 & 0.85 & 0.30 & 1.72 & 0.50 & 0.03 \\
\hline F29 & 2.44 & 1.03 & 1.65 & 0.97 & 0.37 & 1.80 & 0.58 & 0.04 \\
\hline F30 & 1.63 & 0.98 & 1.89 & 0.94 & 0.36 & 1.84 & 0.56 & 0.03 \\
\hline F31 & 1.80 & 1.26 & 1.83 & 1.12 & 0.46 & 2.00 & 0.68 & 0.03 \\
\hline F32 & 1.71 & 1.98 & 2.30 & 1.37 & 0.81 & 2.05 & 0.97 & 0.04 \\
\hline Avg & 1.78 & 0.96 & 1.84 & 1.07 & 0.37 & 2.19 & 0.55 & 0.03 \\
\hline Rank & 6 & 4 & 7 & 5 & 2 & 8 & 3 & 1 \\
\hline
\end{tabular}

Table 22 The comparison results for the gear train design problem

\begin{tabular}{llllll}
\hline Algorithm & \multicolumn{3}{l}{ Optimal values of variables } & Optimal value \\
\cline { 2 - 5 } & $x_{1}$ & $x_{2}$ & $x_{3}$ & $x_{4}$ & \\
\hline MGEHO & 36.83 & 12.04 & 12.26 & 27.76 & $\mathbf{2 . 3 7 E}-\mathbf{1 6}$ \\
EHO & 53 & 19.89 & 16.61 & 35.05 & $1.13 E-03$ \\
EEHO15 & 47.99 & 29.08 & 13.82 & 58.06 & $1.64 E-12$ \\
OEHO & 52.7 & 15.37 & 14.83 & 25.72 & $5.67 E-04$ \\
LFEHO & 40.57 & 12 & 29.75 & 60 & $5.62 E-06$ \\
IGWO & 56.82 & 14.54 & 25.53 & 45.27 & $4.54 E-14$ \\
EO & 55.62 & 16.71 & 21.27 & 44.31 & $3.09 E-13$ \\
HHO & 50.04 & 23.32 & 14.82 & 47.88 & $1.37 E-15$ \\
SFO & 42.9 & 17.03 & 17.03 & 46.83 & $6.00 E-13$ \\
WOA & 51.15 & 22.45 & 17.97 & 55.90 & $4.83 E-11$ \\
\hline
\end{tabular}

and stable than the competing algorithms. Additionally, MGEHO is applied to three specific engineering practice problems. According to the results, MGEHO obtains better solutions than those of the compared algorithms, verifying the ability of MGEHO to solve engineering optimization problems effectively.

In future work, MGEHO will be applied to multilevel threshold image segmentation and will transformed into a 


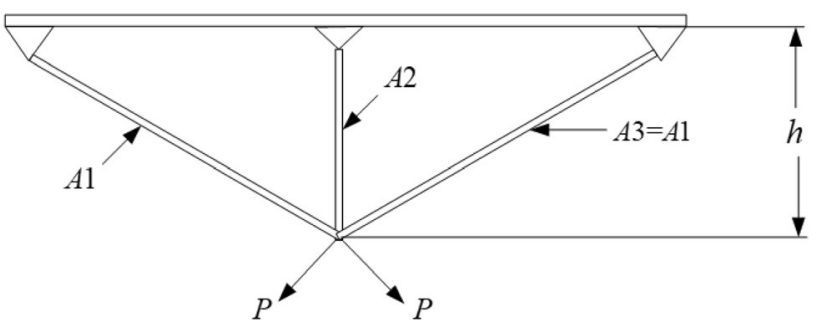

Fig. 6 Design of a three-bar truss

Table 23 The comparison results for the three-bar truss design problem

\begin{tabular}{llll}
\hline Algorithm & \multicolumn{2}{l}{ Optimal values of variables } & \multirow{2}{*}{ Optimal value } \\
\cline { 2 - 3 } & $x_{1}$ & $x_{2}$ & \\
\hline MGEHO & 0.78157 & 0.42853 & $\mathbf{2 6 3 . 8 9 5 7 8}$ \\
EHO & 0.70718 & 0.70718 & 270.73939 \\
EEHO15 & 0.77259 & 0.45579 & 264.10141 \\
OEHO & 0.77329 & 0.5203 & 270.74917 \\
LFEHO & 0.73578 & 0.58431 & 266.54055 \\
IGWO & 0.91039 & 0.31279 & 288.77498 \\
EO & 0.79006 & 0.40433 & 263.89726 \\
HHO & 0.81915 & 0.36956 & 263.89728 \\
SFO & 0.80382 & 0.36702 & 264.05596 \\
WOA & 0.81425 & 0.34033 & 264.33731 \\
\hline
\end{tabular}

Table 24 The comparison results for the pressure vessel design problem

\begin{tabular}{llllll}
\hline \multirow{2}{*}{ Algorithm } & \multicolumn{3}{l}{ Optimal values of variables } & \multirow{2}{*}{ Optimal cost } \\
\cline { 2 - 5 } & $T_{s}$ & $T_{h}$ & $R$ & $L$ & \\
\hline MGEGO & 0.7938 & 0.3879 & 40.6303 & 195.7186 & $\mathbf{5 9 6 6 . 3 7 9 6}$ \\
EEHO15 & 0.8125 & 0.4375 & 42.0984 & 176.6366 & 6059.7143 \\
OEHO & 0.8125 & 0.4375 & 42.0968 & 176.6580 & 6059.9382 \\
LFEHO & 0.8125 & 0.4375 & 42.0912 & 176.7465 & 6061.0777 \\
IGWO & 0.7782 & 0.3847 & 40.3211 & 199.9802 & 5988.0275 \\
EO & 1.1049 & 0.5461 & 57.2487 & 49.5383 & 6711.1442 \\
HHO & 1.0130 & 0.5007 & 52.4910 & 79.7333 & 6420.2814 \\
SFO & 1.1357 & 0.5548 & 58.1465 & 44.4844 & 6833.9158 \\
WOA & 1.1740 & 0.5619 & 58.7656 & 41.1017 & 7001.7002 \\
\hline
\end{tabular}

binary version of a feature selection method. Moreover, MGEHO is also intended to be combined with additional models and methods in machine learning, such as support vector machines, unsupervised learning, artificial neural networks, and convolutional neural networks.
Fig. 7 Design of a pressure vessel

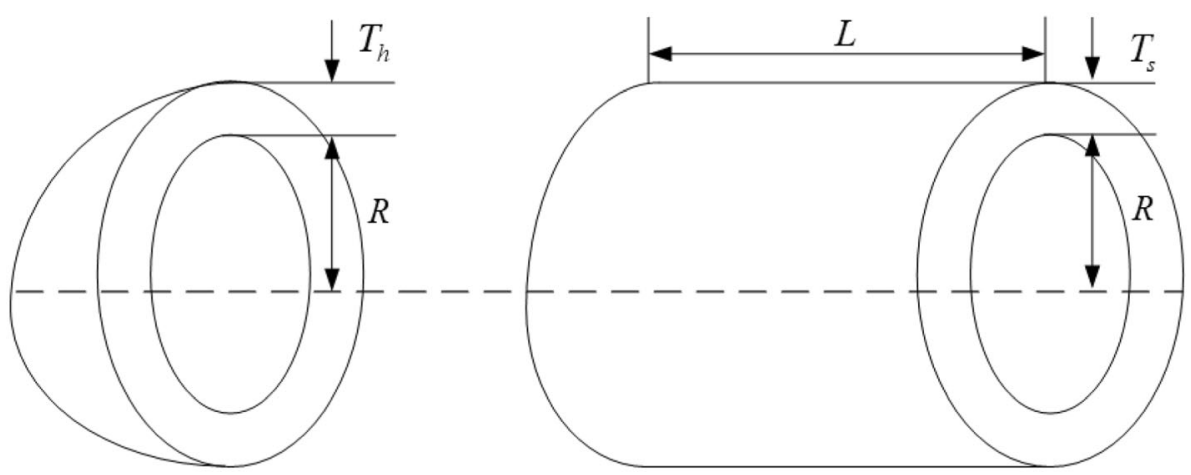




\section{Declarations}

Conflict of interest The authors declare that they have no conflict of interest.

Open Access This article is licensed under a Creative Commons Attribution 4.0 International License, which permits use, sharing, adaptation, distribution and reproduction in any medium or format, as long as you give appropriate credit to the original author(s) and the source, provide a link to the Creative Commons licence, and indicate if changes were made. The images or other third party material in this article are included in the article's Creative Commons licence, unless indicated otherwise in a credit line to the material. If material is not included in the article's Creative Commons licence and your intended use is not permitted by statutory regulation or exceeds the permitted use, you will need to obtain permission directly from the copyright holder. To view a copy of this licence, visit http://creativecommons. org/licenses/by/4.0/.

\section{References}

1. Singh P, Choudhary SK (2021) Introduction: optimization and metaheuristics algorithms. In: Metaheuristic and evolutionary computation: algorithms and applications, vol 916. Springer, pp 3-33

2. Khanduja N, Bhushan B (2021) Recent advances and application of metaheuristic algorithms: a survey (2014-2020). Metaheuristic Evolut Comput 916:207-228

3. Jin Q, Xu Z, Cai W (2021) An improved whale optimization algorithm with random evolution and special reinforcement dualoperation strategy collaboration. Symmetry 13(2):238

4. Huang Y, Shen XN, You X (2021) A discrete shuffled frogleaping algorithm based on heuristic information for traveling salesman problem. Appl Soft Comput 102(2):107085

5. Hussain K, Salleh MNM, Cheng S, Shi Y (2019) Metaheuristic research: a comprehensive survey. Artif Intell Rev 52(4):2191-2233

6. Rizk-Allah RM (2019) An improved sine-cosine algorithm based on orthogonal parallel information for global optimization. Soft Comput 23(16):7135-7161

7. Tang C, Zhou Y, Tang Z, Luo Q (2021) Teaching-learning-based pathfinder algorithm for function and engineering optimization problems. Applied Intelligence 51:5040-5066

8. Holland JH (1992) Genetic algorithms. Sci Am 267(1):66-73

9. Simon D (2008) Biogeography-based optimization. IEEE Trans Evolut Comput 12(6):702-713

10. Storn R, Price K (1997) Differential evolution-a simple and efficient heuristic for global optimization over continuous spaces. J Glob Optimiz 11(4):341-359

11. Bozorg-Haddad O, Janbaz M, Loáiciga HA (2016) Application of the gravity search algorithm to multi-reservoir operation optimization. Adv Water Resour 98:173-185

12. Faramarzi A, Heidarinejad M, Stephens B, Mirjalili S (2020) Equilibrium optimizer: a novel optimization algorithm. KnowlBased Syst 191:105190

13. Karaboga D, Basturk B (2007) A powerful and efficient algorithm for numerical function optimization: artificial bee colony $(a b c)$ algorithm. J Glob Optimiz 39(3):459-471

14. Shadravan S, Naji H, Bardsiri VK (2019) The sailfish optimizer: a novel nature-inspired metaheuristic algorithm for solving constrained engineering optimization problems. Eng Appl Artif Intell 80:20-34

15. Mirjalili S, Lewis A (2016) The whale optimization algorithm. Adv Eng Softw 95:51-67
16. Heidari AA, Mirjalili S, Faris H, Aljarah I, Mafarja M, Chen $\mathrm{H}$ (2019) Harris hawks optimization: algorithm and applications. Future Gener Comput Syst 97:849-872

17. Geem ZW, Kim JH, Loganathan GV (2001) A new heuristic optimization algorithm: harmony search. Simulation 76(2):60-68

18. Rao RV, Savsani VJ, Vakharia D (2011) Teaching-learningbased optimization: a novel method for constrained mechanical design optimization problems. Comput-Aided Design 43(3):303-315

19. Wang GG, Deb S, Gao XZ, Coelho LDS (2016) A new metaheuristic optimisation algorithm motivated by elephant herding behaviour. Int J Bio-Inspired Comput 8(6):394-409

20. Li W, Wang GG (2021) Elephant herding optimization using dynamic topology and biogeography-based optimization based on learning for numerical optimization. Eng Comput 1:1-29

21. Muthusamy H, Ravindran S, Yaacob S, Polat K (2021) An improved elephant herding optimization using sine-cosine mechanism and opposition based learning for global optimization problems. Expert Syst Appl 172:114607

22. Ismaeel AA, Elshaarawy IA, Houssein EH, Ismail FH, Hassanien AE (2019) Enhanced elephant herding optimization for global optimization. IEEE Access 7:34738-34752

23. Elhosseini MA, El Sehiemy RA, Rashwan YI, Gao X (2019) On the performance improvement of elephant herding optimization algorithm. Knowl-Based Syst 166:58-70

24. Balamurugan K, Mahalakshmi R (2020) Anfis-fractional order pid with inspired oppositional optimization based speed controller for brushless dc motor. Int J Wavelets Multiresolut Inf Process 18(01):1941004

25. Xu H, Cao Q, Fang C, Fu Y, Su J, Wei S, Bykovyy P (2018) Application of elephant herd optimization algorithm based on levy flight strategy in intrusion detection. In: 2018 IEEE 4th International Symposium on Wireless Systems within the International Conferences on Intelligent Data Acquisition and Advanced Computing Systems (IDAACS-SWS), IEEE, pp 16-20

26. Li W, Wang GG, Alavi AH (2020) Learning-based elephant herding optimization algorithm for solving numerical optimization problems. Knowl-Based Syst 195:105675

27. Li J, Guo L, Li Y, Liu C (2019) Enhancing elephant herding optimization with novel individual updating strategies for largescale optimization problems. Mathematics 7(5):395

28. Zhao W, Zhang Z, Wang L (2020) Manta ray foraging optimization: an effective bio-inspired optimizer for engineering applications. Eng Appl Artif Intell 87:103300

29. Ekinci S, Izci D, Hekimoğlu B (2021) Optimal fopid speed control of de motor via opposition-based hybrid manta ray foraging optimization and simulated annealing algorithm. Arab J Sci Eng 46(2):1395-1409

30. Shaheen AM, Ginidi AR, El-Sehiemy RA, Ghoneim SS (2020) Economic power and heat dispatch in cogeneration energy systems using manta ray foraging optimizer. IEEE Access 8:208281-208295

31. Ghosh KK, Guha R, Bera SK, Kumar N, Sarkar R (2021) $\mathrm{S}$-shaped versus $\mathrm{v}$-shaped transfer functions for binary manta ray foraging optimization in feature selection problem. Neural Comp Appl 33:11027-11041

32. Liu B, Wang Z, Feng L, Jermsittiparsert K (2021) Optimal operation of photovoltaic/diesel generator/pumped water reservoir power system using modified manta ray optimization. J Clean Prod 289:125733

33. Sheng B, Pan T, Luo Y, Jermsittiparsert K (2020) System identification of the pemfes based on balanced manta-ray foraging optimization algorithm. Energy Rep 6:2887-2896

34. Alturki FA, Omotoso HO, Al-Shamma'a AA, Farh HM, Alsharabi K (2020) Novel manta rays foraging optimization 
algorithm based optimal control for grid-connected pv energy system. IEEE Access 8:187276-187290

35. Wolpert DH, Macready WG (1997) No free lunch theorems for optimization. IEEE Trans Evolut Comput 1(1):67-82

36. Barshandeh S, Haghzadeh M (2020) A new hybrid chaotic atom search optimization based on tree-seed algorithm and levy flight for solving optimization problems. Eng Comput 5:1-44

37. Bäck T, Schwefel HP (1993) An overview of evolutionary algorithms for parameter optimization. Evolut Comput 1(1):1-23

38. Nautiyal B, Prakash R, Vimal V, Liang G, Chen H (2021) Improved salp swarm algorithm with mutation schemes for solving global optimization and engineering problems. Eng Comput 4:1-23

39. Yu C, Cai Z, Ye X, Wang M, Zhao X, Liang G, Chen H, Li C (2020) Quantum-like mutation-induced dragonfly-inspired optimization approach. Math Comput Simul 178:259-289

40. Gupta S, Deep K, Mirjalili S (2020) An efficient equilibrium optimizer with mutation strategy for numerical optimization. Appl Soft Comput 96:106542

41. Nadimi-Shahraki MH, Taghian S, Mirjalili S (2021) An improved grey wolf optimizer for solving engineering problems. Expert Syst Appl 166:113917

42. García S, Fernández A, Luengo J, Herrera F (2010) Advanced nonparametric tests for multiple comparisons in the design of experiments in computational intelligence and data mining: Experimental analysis of power. Inf Sci 180(10):2044-2064

43. Derrac J, García S, Molina D, Herrera F (2011) A practical tutorial on the use of nonparametric statistical tests as a methodology for comparing evolutionary and swarm intelligence algorithms. Swarm Evolut Comput 1(1):3-18

44. García S, Molina D, Lozano M, Herrera F (2009) A study on the use of non-parametric tests for analyzing the evolutionary algorithms' behaviour: a case study on the cec'2005 special session on real parameter optimization. J Heuristics 15(6):617-644

45. Ahmadianfar I, Heidari AA, Gandomi AH, Chu X, Chen $\mathrm{H}$ (2021) Run beyond the metaphor: an efficient optimization algorithm based on runge kutta method. Expert Syst Appl 181:115079

46. Ahmadianfar I, Bozorg-Haddad O, Chu X (2020) Gradient-based optimizer: a new metaheuristic optimization algorithm. Inf Sci 540:131-159
47. Yang Y, Chen H, Heidari AA, Gandomi AH (2021) Hunger games search: visions, conception, implementation, deep analysis, perspectives, and towards performance shifts. Expert Syst Appl 177:114864

48. Li S, Chen H, Wang M, Heidari AA, Mirjalili S (2020) Slime mould algorithm: a new method for stochastic optimization. Future Gener Comput Syst 111:300-323

49. Li C, Li J, Chen H, Heidari AA (2021) Memetic harris hawks optimization: developments and perspectives on project scheduling and qos-aware web service composition. Expert Syst Appl 171:114529

50. Nadimi-Shahraki MH, Taghian S, Mirjalili S, Faris H (2020) Mtde: an effective multi-trial vector-based differential evolution algorithm and its applications for engineering design problems. Appl Soft Comput 97:106761

51. Tu J, Chen H, Liu J, Heidari AA, Zhang X, Wang M, Ruby R, Pham QV (2021) Evolutionary biogeography-based whale optimization methods with communication structure: towards measuring the balance. Knowl-Based Syst 212:106642

52. Holm S (1979) A simple sequentially rejective multiple test procedure. Scand J Stat 6(2):65-70

53. Coello CAC (2002) Theoretical and numerical constraint-handling techniques used with evolutionary algorithms: a survey of the state of the art. Comput Methods Appl Mech Eng 191(11-12):1245-1287

54. Chen C, Wang X, Yu H, Zhao N, Wang M, Chen H (2020) An enhanced comprehensive learning particle swarm optimizer with the elite-based dominance scheme. Complexity 2020(4968063):1-24

55. Sandgren E (1990) Nonlinear integer and discrete programming in mechanical design optimization. J Mech Des 112(2):223-229

56. Kannan B, Kramer SN (1994) An augmented Lagrange multiplier based method for mixed integer discrete continuous optimization and its applications to mechanical design. J Mech Des 116(2):405-411

Publisher's Note Springer Nature remains neutral with regard to jurisdictional claims in published maps and institutional affiliations. 